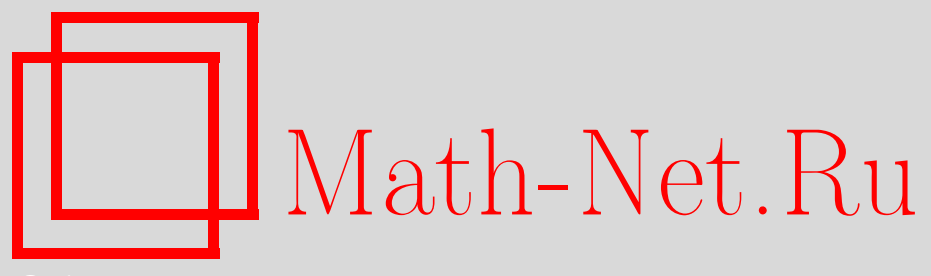

А. Ю. Колесов, Н. Х. Розов, В. А. Садовничий, Математические аспекты теории развития турбулентности по Ландау, УМН, 2008, том 63, выпуск 2, 21-84

DOI: https://doi.org/10.4213/rm9171

Использование Общероссийского математического портала Math-Net.Ru подразумевает, что вы прочитали и согласны с пользовательским соглашением http://www . mathnet.ru/rus/agreement

Параметры загрузки:

IP : 54.197 .130 .99

26 апреля 2023 г., 06:49:42

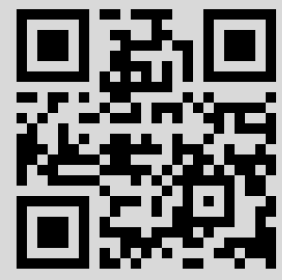




\title{
Математические аспекты теории развития турбулентности по Ландау
}

\author{
А. Ю. Колесов, Н.Х. Розов, В. А. Садовничий
}

В работе представлен ряд строгих математических результатов, связанных с теорией развития турбулентности по Ландау. А именно, рассмотрены конкретные примеры нелинейных динамических систем из различных областей естествознания (в том числе и классический пример Э. Хопфа), аттракторами которых при подходящем изменении параметров оказываются инвариантные торы сколь угодно высоких размерностей. Анализ этих примеров в некоторых случаях позволяет придать строгий смысл понятию “турбулентный аттрактор" и выявить основные свойства такого аттрактора, среди которых следует отметить фрактальность и бесконечномерность.

Библиография: 40 названий.

\section{СоДЕРЖАНИЕ}

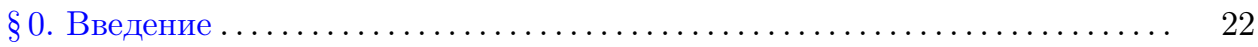

$\S 1$. Базовая модель турбулентности по Ландау .................... 26

1.1. Описание и общие свойства модели .................... 26

1.2. Нормальная форма и ее аттракторы..................... 29

1.3. Итоговые выводы ................................. 38

$\S 2$. Турбулентные структуры на поверхности мелкой воды ........... 45

2.1. Разрешимость начально-краевой задачи ................ 45

2.2. Существование и устойчивость инвариантных торов .......... 51

2.3. Заключительные замечания........................... 55

$\S 3$. Турбулентная экономическая динамика ...................... 60

3.1. Вывод математической модели ....................... 60

3.2. Общие свойства рассматриваемой краевой задачи ........... 62

3.3. Основной результат .............................. 64

3.4. Существование турбулентного аттрактора................ 67

Работа выполнена при поддержке РФФИ (грант № 05-01-01004) и целевой программы "Развитие научного потенциала высшей школы" (проект РНП.2.1.1.630).

(C) А. Ю. Колесов, Н. Х. Розов, В.А. САдовничий, 2008 
$\S 4$. Турбулентная буферность и ее математические модели ............. 70

4.1. Физическая постановка проблемы ............................... 70

4.2. Существование и устойчивость инвариантных торов .......... 73

4.3. О других моделях турбулентной буферности ............... 78

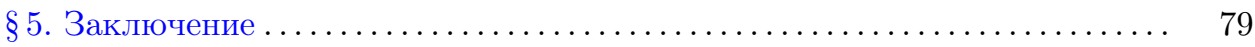

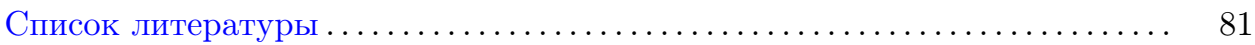

\section{§ 0. Введение}

Динамическая теория развития турбулентности берет свое начало с классических работ Л.Д. Ландау [1] и Э. Хопфа [2]. В первой из этих работ была выдвинута гипотеза о том, что возникновение турбулентности связано с последовательным усложнением динамики за счет появления устойчивых инвариантных торов все более высоких размерностей с квазипериодической обмоткой. Однако до выхода в свет статьи [2] оставался открытым вопрос о принципиальной реализуемости такого сценария.

В работе [2] Э. Хопфу удалось построить достаточно простой пример динамической системы, в которой наблюдается описанный в [1] каскад бифуркаций инвариантных торов. Он предположил, что одномерное течение в замкнутом контуре моделируется параболической краевой задачей вида

$$
\begin{aligned}
u_{t}=-v \circ \bar{v}-u \circ 1+\mu u_{x x}, & v_{t}=v \circ \bar{u}+v \circ \bar{f}(x)+\mu v_{x x}, \\
u(t, x+2 \pi) \equiv u(t, x), & v(t, x+2 \pi) \equiv v(t, x) .
\end{aligned}
$$

Здесь $\mu>0$ - бифуркационный параметр (1/ $\mu$ представляет собой аналог числа Рейнольдса), $u(t, x), v(t, x)$ - комплекснозначные функции, черта означает комплексное сопряжение, оператор о, определенный для любых двух $2 \pi$-периодических функций $f_{1}(x), f_{2}(x)$ посредством равенства

$$
f_{1} \circ f_{2}=\frac{1}{2 \pi} \int_{0}^{2 \pi} f_{1}(x+y) f_{2}(y) d y,
$$

характеризует нелинейное взаимодействие между различными возмущениями, а поле внешней силы $f(x)$ представляет собой четную $2 \pi$-периодическую комплекснозначную функцию класса $L_{2}(-\pi, \pi)$, допускающую разложение Фурье вида

$$
f(x)=a_{0}+i b_{0}+2 \sum_{n=1}^{\infty}\left(a_{n}+i b_{n}\right) \cos n x, \quad a_{n}, b_{n} \in \mathbb{R}, \quad n \geqslant 0 .
$$

Отдельно остановимся на ограничениях, накладываемых на фигурирующие в (0.3) коэффициенты $a_{n}, b_{n}, n \geqslant 0$. Несколько усиливая предположения работы [2], будем считать, что

$$
a_{0}<0, \quad a_{1}>a_{2} / 4>\cdots>a_{n} / n^{2}>a_{n+1} /(n+1)^{2}>\cdots>0
$$

(напомним, что в [2] требовалось лишь выполнение условия $a_{0}<0$, а также неравенств $a_{n}>0$ для бесконечно большого числа номеров $n$ ). Что же касается 
коэффициентов $b_{n}, n \geqslant 1$, то, как и в [2], предполагаем, что любое их конечное семейство линейно независимо над полем рациональных чисел.

При сформулированных условиях будем рассматривать краевую задачу (0.1) как динамическую систему в фазовом пространстве $(u, v) \in V \times V$, где $V$ - гильбертово пространство четных $2 \pi$-периодических комплекснозначных функций класса $L_{2}(-\pi, \pi)$. Скалярное произведение в этом пространстве определим следующим образом. Фиксируем произвольно две функции $u(x), v(x)$ из $V$ и разложим их в ряды Фурье

$$
\begin{gathered}
u=\sum_{n=-\infty}^{\infty} u_{n} \exp (\text { inx }), \quad v=\sum_{n=-\infty}^{\infty} v_{n} \exp (\text { inx }), \\
u_{n}=u_{-n}, \quad v_{n}=v_{-n}, \quad n \geqslant 1
\end{gathered}
$$

(последние два равенства - следствие четности $u(x), v(x)$ ). Тогда по определению

$$
\begin{gathered}
(u, v)_{V}=u_{0} \bar{v}_{0}+2 \sum_{n=1}^{\infty} u_{n} \bar{v}_{n}, \\
\left(\left(u_{1}, v_{1}\right),\left(u_{2}, v_{2}\right)\right)_{V \times V}=\left(u_{1}, u_{2}\right)_{V}+\left(v_{1}, v_{2}\right)_{V} \quad \forall\left(u_{1}, v_{1}\right),\left(u_{2}, v_{2}\right) \in V \times V .
\end{gathered}
$$

Анализ динамики по параметру $\mu$ краевой задачи (0.1) в пространстве $V \times V$ не вызывает затруднений. Действительно, подставим в (0.1) равенство (0.3) вместе с разложениями (0.5) и учтем вытекающие из (0.2) соотношения

$$
v \circ \bar{v}=\sum_{n=-\infty}^{\infty}\left|v_{n}\right|^{2} \exp (\text { inx }), \quad v \circ \bar{u}=\sum_{n=-\infty}^{\infty} v_{n} \bar{u}_{n} \exp (\text { inx }) .
$$

В результате для определения $u_{n}=u_{n}(t), v_{n}=v_{n}(t), n \geqslant 0$, приходим к счетной системе обыкновенных дифференциальных уравнений, которая распадается на двумерные блоки вида

$$
\begin{gathered}
\dot{u}_{0}=-\left|v_{0}\right|^{2}-u_{0}, \quad \dot{v}_{0}=v_{0} \bar{u}_{0}+\left(a_{0}-i b_{0}\right) v_{0}, \\
\dot{u}_{n}=-\left|v_{n}\right|^{2}-n^{2} \mu u_{n}, \quad \dot{v}_{n}=v_{n} \bar{u}_{n}+\left(a_{n}-i b_{n}\right) v_{n}-n^{2} \mu v_{n}, \quad n \geqslant 1 .
\end{gathered}
$$

В первую очередь обратимся к системе (0.7) и заметим, что из очевидного соотношения

$$
\frac{1}{2} \frac{d}{d t}\left(\left|u_{0}\right|^{2}+\left|v_{0}\right|^{2}\right)=-\left(\left|u_{0}\right|^{2}-a_{0}\left|v_{0}\right|^{2}\right)
$$

и из условия $a_{0}<0$ (см. (0.4)) вытекает стремление к нулю всех ее решений при $t \rightarrow+\infty$. Далее, фиксируем произвольно номер $n \geqslant 1$ и предположим сначала, что $\mu>\mu_{n}=a_{n} / n^{2}$. Тогда, как нетрудно увидеть,

$$
\frac{1}{2} \frac{d}{d t}\left(\left|u_{n}\right|^{2}+\left|v_{n}\right|^{2}\right)=-\left(n^{2} \mu\left|u_{n}\right|^{2}+\left(n^{2} \mu-a_{n}\right)\left|v_{n}\right|^{2}\right)<0
$$

а значит, аналогичное поведение решений наблюдается и в соответствующей системе (0.8). 
Предположим теперь, что параметр $\mu$ в (0.8) удовлетворяет условию $0<$ $\mu<\mu_{n}$. В этом случае, как показывает непосредственная проверка, у рассматриваемой системы появляется экспоненциально орбитально устойчивый гармонический по форме цикл

$$
u_{n}=n^{2} \mu-a_{n}, \quad v_{n}=\sqrt{n^{2} \mu\left(a_{n}-n^{2} \mu\right)} \exp (i \varphi), \quad \dot{\varphi}=-b_{n} .
$$

Более детальный анализ приводит к следующему утверждению [2].

ТЕОРемА 0.1. При любом $\mu \in\left(0, \mu_{n}\right)$ область притяжсения $\mathscr{U}$ автомодельного иикла (0.9) системы (0.8) задается равенством

$$
\mathscr{U}=\left\{\left(u_{n}, v_{n}\right) \in \mathbb{C}^{2}: v_{n} \neq 0\right\} .
$$

ДокАЗАТЕЛЬство. Для начала выполним в системе (0.8) замены переменных $u_{n}=q+i p, q, p \in \mathbb{R}$, и $v_{n}=r \exp (i \varphi), r \geqslant 0, \varphi \in \mathbb{R}$, в результате которых она преобразуется к виду

$$
\dot{q}=-r^{2}-n^{2} \mu q, \quad \dot{p}=-n^{2} \mu p, \quad \dot{r}=\left(q+a_{n}-n^{2} \mu\right) r, \quad \dot{\varphi}=-b_{n}-p .
$$

Далее заметим, что для компонент $p$ и $\varphi$ получившейся системы $(0.11)$ при $t \rightarrow+\infty$ имеют место предельные равенства $p \rightarrow 0, \dot{\varphi} \rightarrow-b_{n}$. А отсюда следует, что интересующая нас проблема сводится к анализу двумерной системы

$$
\dot{q}=-r^{2}-n^{2} \mu q, \quad \dot{r}=\left(q+a_{n}-n^{2} \mu\right) r .
$$

Точнее говоря, в силу (0.9) нам нужно доказать, что любая ее траектория, для которой $\left.r\right|_{t=0}>0$, при $t \rightarrow+\infty$ стремится к экспоненциально устойчивому состоянию равновесия $(q, r)=\left(n^{2} \mu-a_{n}, \sqrt{n^{2} \mu\left(a_{n}-n^{2} \mu\right)}\right)$.

Поскольку условие $\left.r\right|_{t=0}>0$ влечет выполнение неравенства $r(t)>0$ при всех $t$, то мы имеем право сделать в системе $(0.12)$ замену $r^{2}=\exp (Q)$, приводящую ее к виду

$$
\dot{q}=-\exp (Q)-n^{2} \mu q, \quad \dot{Q}=2\left(q+a_{n}-n^{2} \mu\right) .
$$

После этого выразим $q$ из второго уравнения системы $(0.13)$ и результат подставим в первое уравнение. В итоге приходим к выводу, что $Q$ - решение скалярного уравнения второго порядка

$$
\ddot{Q}+n^{2} \mu \dot{Q}=-H^{\prime}(Q)
$$

где

$$
\begin{aligned}
H(Q) & =2\left(\exp (Q)-\exp \left(Q_{0}\right)-\left(Q-Q_{0}\right) \exp \left(Q_{0}\right)\right), \\
Q_{0} & =\ln \left(n^{2} \mu\left(a_{n}-n^{2} \mu\right)\right) .
\end{aligned}
$$

Для того чтобы убедиться, что область притяжения цикла (0.9) имеет вид (0.10), осталось показать, что любое решение $Q(t)$ уравнения $(0.14)$ обладает свойствами: $Q(t) \rightarrow Q_{0}, \dot{Q}(t) \rightarrow 0$ при $t \rightarrow+\infty$. Для их проверки введем в рассмотрение функцию $w(t)=(\dot{Q}(t))^{2} / 2+H(Q(t))$. Заметим, далее, что в силу вытекающих из (0.15) соотношений

$$
\begin{gathered}
H\left(Q_{0}\right)=H^{\prime}\left(Q_{0}\right)=0, \quad H(Q)>0 \quad \text { при } Q \neq Q_{0}, \\
H(Q) \rightarrow+\infty \quad \text { при }|Q| \rightarrow+\infty, \quad \dot{w}(t)=-n^{2} \mu(\dot{Q}(t))^{2} \leqslant 0
\end{gathered}
$$


для функции $w(t)$ при тех $t \geqslant 0$, для которых она в принципе определена, справедливы априорные оценки $0 \leqslant w(t) \leqslant w(0)$. А отсюда автоматически следует, что на самом деле $w(t)$ существует на полуоси $t \geqslant 0$ и при $t \rightarrow+\infty$ имеет конечный предел $w_{*} \geqslant 0$.

На завершающем этапе доказательства на плоскости $(Q, \dot{Q})$ рассмотрим траекторию $(Q(t), \dot{Q}(t))$ и обозначим через $\Lambda$ ее $\omega$-предельное множество. Из установленных выше свойств функции $w(t)$ заключаем, что $\Lambda \subset\left\{(Q, \dot{Q}): \dot{Q}^{2} / 2+\right.$ $\left.H(Q)=w_{*}\right\}$. Следовательно, для любой траектории $\left(Q_{*}(t), \dot{Q}_{*}(t)\right) \in \Lambda$ выполняется тождество $\left(\dot{Q}_{*}(t)\right)^{2} / 2+H\left(Q_{*}(t)\right) \equiv w_{*}$, дифференцируя которое по $t$, приходим к выводу (см. последнее равенство из $(0.16))$, что $\dot{Q}_{*}(t) \equiv 0$.

Итак, установлено, что в множество $\Lambda$ могут входить только состояния равновесия $\left(Q_{*}(t), \dot{Q}_{*}(t)\right) \equiv$ (const, 0$)$ уравнения (0.14). Остается заметить, что это уравнение допускает единственное состояние равновесия $\left(Q_{0}, 0\right)$, где величина $Q_{0}$ определена в (0.15). Теорема 0.1 доказана.

Суммируя проделанные построения, приходим к выводу, что исходная система (0.1) имеет по параметру $\mu$ следующую динамику. Пусть сначала $\mu>\mu_{1}$. Тогда в силу (0.4) будут выполняться и неравенства $\mu>\mu_{n}$ при $n \geqslant 2$. А отсюда и из свойств систем $(0.7),(0.8)$ заключаем, что в этом случае нулевое состояние равновесия краевой задачи (0.1) глобально экспоненциально устойчиво в пространстве $V \times V$ (в метрике, порожденной скалярным произведением из $(0.6))$.

Пусть теперь $\mu_{n+1}<\mu<\mu_{n}$ при некотором $n \geqslant 1$. Тогда в первых $n$ системах семейства (0.8) наблюдаются устойчивые циклы вида (0.9), а в остальных по-прежнему глобально устойчиво нулевое решение. В терминах исходной задачи (0.1) это означает, что в пространстве $V \times V$ у нее существует экспоненциально орбитально устойчивый $n$-мерный инвариантный тор:

$$
\begin{gathered}
u=2 \sum_{k=1}^{n}\left(k^{2} \mu-a_{k}\right) \cos k x, \\
T_{n}: \quad v=2 \sum_{k=1}^{n} \sqrt{k^{2} \mu\left(a_{k}-k^{2} \mu\right)} \exp \left(i \varphi_{k}\right) \cos k x, \\
\dot{\varphi}_{k}=-b_{k}, \quad k=1, \ldots, n,
\end{gathered}
$$

где $0 \leqslant \varphi_{k} \leqslant 2 \pi(\bmod 2 \pi), k=1, \ldots, n,-$ отвечающие этому тору циклические координаты. Заметим, далее, что в силу наложенных на $b_{k}, k=1, \ldots, n$, ограничений движения на торе (0.17) являются квазипериодическими. Кроме того, как следует из теоремы 0.1 , при $\mu_{n+1}<\mu<\mu_{n}$ он оказывается единственным аттрактором системы (0.1), притягивающим любые ее траектории с начальными условиями

$$
(u(0, x), v(0, x)) \in V \times V: \quad \int_{0}^{2 \pi} v(0, x) \cos k x d x \neq 0, \quad k=1, \ldots, n .
$$

При дальнейшем уменьшении параметра $\mu$ и при прохождении его через критическое значение $\mu=\mu_{n+1}$ тор (0.17) теряет устойчивость и порождает аналогичный устойчивый тор $T_{n+1}$ на единицу большей размерности. Таким 
образом, имеем бесконечную последовательность бифуркационных значений $\mu_{n} \searrow 0, n \rightarrow \infty$, и инвариантных торов $T_{n}, n \geqslant 1$, с квазипериодической обмоткой. А это, собственно, и означает, что при $\mu \rightarrow 0$ в рамках краевой задачи (0.1) реализуется сценарий Л. Д. Ландау.

Гипотеза Л. Д. Ландау сыграла большую роль в процессе осознания природы турбулентности. В частности, развитые Л. Д. Ландау представления легли в основу известных гипотез А.Н. Колмогорова о росте размерности аттракторов уравнений Навье-Стокса при увеличении числа Рейнольдса (точные формулировки этих гипотез приведены, например, в [3]). Однако современные представления о турбулентности сложились во многом благодаря открытию феномена динамического хаоса [4]. K настоящему времени выработано несколько различных эвристических сценариев перехода к турбулентности, среди которых следует отметить разрушение квазипериодических движений [5], [6] (точнее говоря, в указанных работах Д. Рюэль и Ф. Такенс предложили сценарий, когда инвариантные торы превращаются после конечного числа шагов в гиперболические аттракторы), перемежаемость [7] и бесконечную последовательность бифуркаций удвоения периода [8]. Что же касается сценария Ландау, то его нельзя отнести к числу основных, поскольку аттрактор в виде незамкнутой плотной намотки на многомерном торе структурно неустойчив, т. е. он разрушается при малом изменении параметров системы. Тем не менее, усложнение динамики по Ландау все же реализуется в некоторых специальных случаях. В качестве примера сошлемся на статью [9], где рассматривалась цепочка однонаправленно связанных ротаторов.

В настоящей работе мы пытаемся отчасти реабилитировать сценарий Ландау путем отказа от требования квазипериодичности движений на возникающих инвариантных торах (аналогичную идею ранее высказывал Дж. Селл в статье [10]). Как будет установлено ниже, в таком расширенном варианте этот сценарий наблюдается в ряде примеров из различных областей естествознания.

\section{§ 1. Базовая модель турбулентности по Ландау}

1.1. Описание и общие свойства модели. Разобранный выше пример Хопфа имеет по крайней мере два существенных недостатка. Во-первых, хотя при $\mu \rightarrow 0$ количество мод $\exp ( \pm i n x), n \geqslant 1$, вовлеченных в нелинейное взаимодействие, неограниченно растет, но само это взаимодействие носит блочный характер (см. (0.7), (0.8)). На наш взгляд, более адекватным проблеме было бы взаимодействие мод по типу среднего поля. Во-вторых, при $\mu=0$, т. е. в случае нулевой вязкости, когда аттрактор, в принципе, должен стать бесконечномерным (развитая турбулентность), в системе (0.1) происходит его вырождение в состояние равновесия $u=-2 \sum_{k=1}^{\infty} a_{k} \cos k x, v=0$, что непосредственно вытекает из формул (0.17). Следует отметить, впрочем, что сценарий Ландау относится к возникновению турбулентности, а не к развитой турбулентности. Последняя же описывается асимптотиками решений уравнения Навье-Стокса, когда вязкость стремится к нулю. Тем не менее, было бы интересно скорректировать модель Хопфа таким образом, чтобы объединить эти два феномена. 
Один из возможных способов ее модификации предлагается в настоящем параграфе.

Как и в [2], рассмотрим одномерное течение в замкнутом контуре, но теперь будем считать, что оно описывается одной вещественной скалярной функцией $u(t, x)$, удовлетворяющей следующей краевой задаче:

$$
u_{t}-\nu u_{x x}=\mathscr{L}_{0} u+F(u), \quad u(t, x+2 \pi) \equiv u(t, x), \quad M(u) \equiv 0 .
$$

Здесь $\nu \geqslant 0$ - бифуркационный параметр, характеризующий вязкость среды, а операторы $\mathscr{L}_{0}$ и $F$ действуют на произвольную $2 \pi$-периодическую функцию $u(x)$ по правилам:

$$
\begin{gathered}
\mathscr{L}_{0} u=f(x) * u(x), \quad F(u)=\left(1-M\left(u^{2}\right)\right) u(x)-u(x) * u(x) * u(-x), \\
M(u)=\frac{1}{2 \pi} \int_{0}^{2 \pi} u(x) d x \\
u_{1}(x) * u_{2}(x)=\frac{1}{2 \pi} \int_{0}^{2 \pi} u_{1}(x-y) u_{2}(y) d y \quad \forall u_{1}(x), u_{2}(x) .
\end{gathered}
$$

Что же касается поля внешней силы $f(x)$, то в данном случае оно задается некоторым формальным рядом вида

$$
f(x)=\sum_{n=1}^{\infty}\left(i \sigma_{n} \exp (i n x)-i \sigma_{n} \exp (-i n x)\right),
$$

где $\sigma_{n}>0, n \geqslant 1,-$ произвольно фиксированная числовая последовательность, удовлетворяющая двум условиям: $\sigma_{n} \nearrow \infty$ при $n \rightarrow \infty$ и при любом $n \geqslant 1$ семейство $\sigma_{k}, 1 \leqslant k \leqslant n$, линейно независимо над полем рациональных чисел.

В качестве фазового пространства краевой задачи (1.1) возьмем банахово пространство $E_{0}$, состоящее из вещественных скалярных $2 \pi$-периодических функций $u(x)$ класса $L_{2}(-\pi, \pi)$ с нулевым средним значением. Норму в $E_{0}$ определим формулой

$$
\|u\|=\left(2 \sum_{n=1}^{\infty}\left|z_{n}\right|^{2}\right)^{1 / 2},
$$

где $z_{n} \in \mathbb{C}, n \geqslant 1,-$ коэффициенты разложения Фурье

$$
u=\sum_{n=1}^{\infty}\left(z_{n} \exp (i n x)+\bar{z}_{n} \exp (-i n x)\right) .
$$

Далее, заметим, что равенства (1.4), (1.5) осуществляют естественный изоморфизм между $E_{0}$ и линейным пространством $Z_{0}$, состоящим из бесконечномерных векторов

$$
z=\left(z_{1}, \bar{z}_{1}, z_{2}, \bar{z}_{2}, \ldots, z_{n}, \bar{z}_{n}, \ldots\right), \quad z_{n} \in \mathbb{C}, \quad n \geqslant 1,
$$

для которых конечна норма (1.4). Подчеркнем, что пространство $Z_{0}$ следует рассматривать над полем действительных чисел, так как только в этом случае при умножении его элементов на скаляры снова будут получаться векторы вида (1.6). 
На следующем этапе введем понятие решения краевой задачи (1.1). Для этого нам потребуется линейный оператор $\mathscr{L}_{\nu}=\mathscr{L}_{0}+\nu d^{2} / d x^{2}, \nu \geqslant 0$. Привлекая равенство (1.3), а также справедливую для любых двух функций $u(x), v(x)$ из $E_{0}$ формулу

$$
u(x) * v(x)=\sum_{n=1}^{\infty}\left(u_{n} v_{n} \exp (i n x)+\bar{u}_{n} \bar{v}_{n} \exp (-i n x)\right)
$$

где

$$
\begin{gathered}
u(x)=\sum_{n \neq 0} u_{n} \exp (\text { inx }), \quad v(x)=\sum_{n \neq 0} v_{n} \exp (\text { inx }), \\
u_{-n}=\bar{u}_{n}, \quad v_{-n}=\bar{v}_{n}, \quad n \geqslant 1 ;
\end{gathered}
$$

нетрудно убедиться, что $\mathscr{L}_{\nu}$ представляет собой замкнутый неограниченный линейный оператор с плотной в $E_{0}$ областью определения, действующий на произвольную функцию (1.5) по правилу

$$
\mathscr{L}_{\nu} u=\sum_{n=1}^{\infty}\left(\left(i \sigma_{n}-n^{2} \nu\right) z_{n} \exp (i n x)+\left(-i \sigma_{n}-n^{2} \nu\right) \bar{z}_{n} \exp (-i n x)\right) .
$$

А отсюда немедленно следует, что он порождает в $E_{0}$ сильно непрерывную по $t \geqslant 0$ полугруппу $\exp \left(\mathscr{L}_{\nu} t\right)$ линейных ограниченных операторов, для которой справедлива аналогичная (1.9) формула

$$
\exp \left(\mathscr{L}_{\nu} t\right) u=\sum_{n=1}^{\infty}\left(z_{n} \exp \left[i\left(\sigma_{n} t+n x\right)-n^{2} \nu t\right]+\bar{z}_{n} \exp \left[-i\left(\sigma_{n} t+n x\right)-n^{2} \nu t\right]\right) .
$$

Проделанные предварительные построения позволяют определить обобщенные решения задачи (1.1) следующим образом. Дополним (1.1) произвольным начальным условием $u(0, x)=u_{0}(x) \in E_{0}$ и с помощью полугруппы (1.10) перейдем к соответствующему интегральному уравнению

$$
u(t, x)=\exp \left(\mathscr{L}_{\nu} t\right) u_{0}(x)+\int_{0}^{t} \exp \left(\mathscr{L}_{\nu}(t-\tau)\right) F(u(\tau, x)) d \tau .
$$

Под обобщенным решением смешанной задачи для (1.1) с выбранным начальным условием будем понимать непрерывное по $t$ в метрике пространства $E_{0}$ решение $u=u(t, x)$ этого интегрального уравнения.

Отметим, что введенное таким способом понятие решения корректно в том смысле, что для него справедливы свойства локального существования и единственности. Действительно, опираясь на факт ограниченности и гладкости по Фреше оператора $F: E_{0} \rightarrow E_{0}$ (см. (1.2)), несложно проверить, что оператор, порожденный в пространстве $C\left(\left[0, t_{0}\right] ; E_{0}\right)$ правой частью уравнения $(1.11)$, при подходящем уменьшении $t_{0}>0$ преобразует в себя некоторый замкнутый шар этого пространства и является сжимающим. А отсюда и из принципа сжимающих отображений вытекает существование при $0 \leqslant t \leqslant t_{0}$ единственного обобщенного решения задачи (1.1) с рассматриваемым начальным условием.

Добавим еще, что при $\nu>0$ краевая задача (1.1) является параболической и в силу известных свойств параболических уравнений (см., например, [11], [12]) 
мы можем утверждать, что в этом случае любое ее обобщенное решение автоматически оказывается классическим. Точнее говоря, при $t>0$ оно имеет любое фиксированное число непрерывных производных по $t, x$ и обращает уравнение из (1.1) в тождество. В случае же $\nu=0$, т. е. при нулевой вязкости, сглаживания решений по $x$ при $t>0$ не происходит и подавляющее большинство из них так и остаются обобщенными.

1.2. Нормальная форма и ее аттракторы. Для удобства последующего анализа сначала перейдем от краевой задачи (1.1) к эквивалентной системе в пространстве $Z_{0}$. Используя формулы (1.5) и (1.7)-(1.9), нетрудно увидеть, что для компонент $z_{n}=z_{n}(t), n \geqslant 1$, вектора (1.6) на этом пути получаем систему

$$
\dot{z}_{n}=\left[1+i \sigma_{n}-\nu n^{2}-3\left|z_{n}\right|^{2}-2 \sum_{\substack{k=1 \\ k \neq n}}^{\infty}\left|z_{k}\right|^{2}\right] z_{n}, \quad n \geqslant 1,
$$

которая в отличие от (0.7), (0.8) уже не распадается ни на какие конечномерные блоки. Тем самым, здесь реализуется требуемое взаимодействие мод по принципу среднего поля.

Далее, полагая в (1.12)

$$
z_{n}=\sqrt{\rho_{n}} \exp \left(i \varphi_{n}\right), \quad \rho_{n} \geqslant 0, \quad \varphi_{n} \in \mathbb{R}, \quad n \geqslant 1,
$$

убеждаемся, что переменные $\rho_{n}, \varphi_{n}$ в свою очередь удовлетворяют системе

$$
\dot{\rho}_{n}=2\left[1-\nu n^{2}-3 \rho_{n}-2 \sum_{\substack{k=1 \\ k \neq n}}^{\infty} \rho_{k}\right] \rho_{n}, \quad \dot{\varphi}_{n}=\sigma_{n}, \quad n \geqslant 1 .
$$

И наконец, рассмотрим отщепляющуюся от (1.14) систему для амплитудных переменных $\rho_{n}, n \geqslant 1$, которая после замены $2 t \rightarrow t$ принимает вид

$$
\dot{\rho}_{n}=\left[1-\nu n^{2}-3 \rho_{n}-2 \sum_{\substack{k=1 \\ k \neq n}}^{\infty} \rho_{k}\right] \rho_{n}, \quad n \geqslant 1 .
$$

Ясно, что в конечном итоге именно она и отвечает за возможные аттракторы исходной задачи (1.1). В связи с этим в дальнейшем за ней закрепим термин "нормальная форма".

В силу (1.4), (1.5), (1.13) в качестве фазового пространства нормальной формы следует взять пространство $l$, элементами которого являются векторы

$$
\rho=\left(\rho_{1}, \rho_{2}, \ldots, \rho_{n}, \ldots\right):\|\rho\|=\sum_{n=1}^{\infty}\left|\rho_{n}\right|<\infty .
$$

Точнее говоря, будем рассматривать ее в инвариантном конусе $K \subset l$, состоящем из векторов (1.16) с неотрицательными координатами.

Установим сначала некоторые общие свойства решений системы (1.15). С этой целью зафиксируем произвольно вектор $\rho_{0}=\left(\rho_{1}^{0}, \ldots, \rho_{n}^{0}, \ldots\right) \in K$ и обо- 
значим через $\rho(t)=\left(\rho_{1}(t), \ldots, \rho_{n}(t), \ldots\right)$ ее траекторию с начальным условием $\rho(0)=\rho_{0}$. Рассмотрим, далее, функцию

$$
S(t)=\sum_{n=1}^{\infty} \rho_{n}(t)
$$

и заметим, что она является непрерывно дифференцируемой при тех значениях $t>0$, для которых существует траектория $\rho(t)$ (в силу сказанного в предыдущем пункте множество таких $t$ заведомо не пусто).

Действительно, при $\nu>0$ гладкость функции (1.17) - следствие параболичности нормальной формы (согласно [13] она представляет собой абстрактную полулинейную параболическую систему в пространстве $l$ ). Если же $\nu=0$, то правая часть из (1.15) является ограниченным и гладким по Фреше нелинейным оператором, действующим из $l$ в $l$, а значит, мы можем воспользоваться результатами монографии [14] и установить в этом случае гладкость $S(t)$ вплоть до точки $t=0$.

Факт дифференцируемости $S(t)$ и вытекающее из структуры правых частей нормальной формы дифференциальное неравенство

$$
\dot{S}(t) \leqslant S(t)-2 S^{2}(t)
$$

приводят к априорной оценке

$$
S(t) \leqslant S_{*}(t), \quad t \geqslant 0
$$

где $S_{*}(t)$ - решение задачи Коши

$$
\dot{S}=S-2 S^{2},\left.\quad S\right|_{t=0}=S_{0}=\sum_{n=1}^{\infty} \rho_{n}^{0} .
$$

А отсюда и из известных свойств полулинейных параболических уравнений (см. теорему 3.3 .4 из [13]) следует продолжимость рассматриваемой траектории $\rho(t)$ системы (1.15) на всю полуось $t \geqslant 0$. Таким образом, нормальная форма порождает в конусе $K$ нелинейный полупоток $g^{t}\left(\rho_{0}\right), \rho_{0} \in K$, определенный по правилу

$$
g^{t}\left(\rho_{0}\right)=\rho(t), \quad t \in \mathbb{R}_{+} .
$$

При анализе полупотока (1.21) нам потребуется множество $\mathscr{U}=K \cap\{\rho$ : $\|\rho\| \leqslant 1\}$. Из очевидного неравенства $\dot{S}(t) \leqslant-1$ при $S(t) \geqslant 1$ (см. (1.18)) заключаем, что это множество является поглощающим. Последнее означает существование для любого ограниченного подмножества $\widetilde{K} \subset K$ такого момента времени $t_{0}=t_{0}(\widetilde{K})>0$, что $g^{t}(\widetilde{K}) \subset \mathscr{U}$ при всех $t \geqslant t_{0}$. Ясно также, что наличие инвариантного поглощающего множества автоматически влечет существование у полупотока (1.21) в конусе $K$ глобального аттрактора

$$
\widetilde{A}_{\nu}=\bigcap_{t \geqslant 0} g^{t}(\mathscr{U})
$$


Наша основная задача состоит в изучении структуры аттрактора (1.22) при различных значениях параметра $\nu \geqslant 0$, включая случай $\nu=0$. Для формулировки соответствующего результата введем в рассмотрение монотонно убывающую последовательность

$$
\bar{\nu}_{N}=\frac{3}{N\left(4 N^{2}-1\right)}, \quad N \geqslant 1 .
$$

Справедливо следующее утверждение.

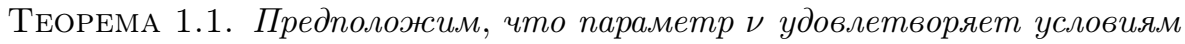

$$
\bar{\nu}_{N+1}<\nu<\bar{\nu}_{N}
$$

при некотором $N \geqslant 1$. Тогда аттрактор (1.22) содержит экспоненциально устойчивое состояние равновесия $O_{N}$ с координатами

$$
\begin{array}{ll}
\rho_{n}=\frac{1}{2 N+1}+\frac{\nu}{3}\left(N(N+1)-3 n^{2}\right) & \text { nрu } n=1, \ldots, N \\
\rho_{n}=0 & \text { nрu } n \geqslant N+1 .
\end{array}
$$

Все же остальные возможные состояния равновесия, входящие в $\widetilde{A}_{\nu}$, экспоненциально неустойчивъ.

ДокАЗАТЕльство. Фиксируем произвольно натуральное $N$ и положим

$$
K_{N}=\left\{\left(\rho_{1}, \ldots, \rho_{n}, \ldots\right) \in l: \rho_{n}>0, n=1, \ldots, N ; \rho_{n}=0 \forall n \geqslant N+1\right\} .
$$

Как показывает непосредственная проверка, при всех $\nu \in\left[0, \bar{\nu}_{N}\right)$ система $(1.15)$ имеет в множестве (1.26) единственное состояние равновесия с координатами $(1.25)$, которое и обозначим через $O_{N}$. Добавим еще, что при $\nu=\bar{\nu}_{N}$ оно "влипает" в аналогичное состояние равновесия $O_{N-1} \subset K_{N-1}$, поскольку в этом случае обращается в нуль компонента $\rho_{N}$ из (1.25), а все остальные координаты $\rho_{n}, n=1, \ldots, N-1$, остаются положительными и совпадают с компонентами неподвижной точки $O_{N-1}$.

Убедимся теперь, что состояние равновесия $O_{N}$ экспоненциально устойчиво при выполнении неравенств (1.24) и неустойчиво при $\nu \in\left[0, \bar{\nu}_{N+1}\right)$. С этой целью линеаризуем на нем систему (1.15) и заметим, что получившаяся система в вариациях имеет блочно-диагональную структуру. Точнее говоря, она распадается на систему из $N$ уравнений

$$
\dot{h}=-B h, \quad h=\operatorname{colon}\left(h_{1}, \ldots, h_{N}\right),
$$

где элементы $b_{j, r}$ матрицы $B$ задаются равенствами

$$
b_{j, j}=3 \rho_{j}, \quad b_{j, r}=2 \rho_{j}, \quad j, r=1, \ldots, N, \quad j \neq r
$$

и на серию скалярных уравнений

$$
\dot{h}_{m}=\left(1-2 \sum_{k=1}^{N} \rho_{k}-\nu m^{2}\right) h_{m}, \quad m \geqslant N+1 .
$$


Анализ свойств устойчивости системы (1.27) сводится, очевидно, к исследованию расположения корней уравнения

$$
\operatorname{det}(\lambda I+B)=0
$$

где $I$ - единичная матрица. Покажем, что все они вещественны и отрицательны.

Для того чтобы раскрыть определитель в уравнении (1.29), сначала вычтем его последний столбец из всех предыдущих. После этого добавим к последней строке получившегося определителя все остальные его строки, каждая из которых умножена соответственно на

$$
\frac{\rho_{N}+\lambda}{\rho_{j}+\lambda}, \quad j=1, \ldots, N-1 .
$$

В итоге уравнение (1.29) преобразуется к более удобному для последующего анализа виду

$$
\left(\rho_{1}+\lambda\right) \cdots\left(\rho_{N}+\lambda\right)\left[1+\sum_{j=1}^{N} \frac{2 \rho_{j}}{\rho_{j}+\lambda}\right]=0 .
$$

Нетрудно заметить, что в силу вытекающих из (1.25) неравенств $\rho_{n}>\rho_{n+1}$, $n=1, \ldots, N-1$, числа $\lambda=-\rho_{j}, j=1, \ldots, N$, не являются решениями уравнения (1.30), а значит, оно эквивалентно уравнению

$$
\sum_{j=1}^{N} \frac{\rho_{j}}{\rho_{j}+\lambda}=-\frac{1}{2}
$$

которое имеет на полуоси $\lambda<0$ ровно $N$ различных корней, так как входящая в левую часть (1.31) функция на интервалах $\left(-\rho_{j},-\rho_{j+1}\right), j=1, \ldots, N-1$, и $\left(-\infty,-\rho_{1}\right)$ монотонно меняется в пределах от $-\infty$ до $+\infty$ и от 0 до $-\infty$ соответственно.

Анализ свойств устойчивости уравнений (1.28) тривиален. Действительно, опираясь на формулы (1.23), (1.25), приходим к выводу, что

$$
\max _{m \geqslant N+1}\left(1-2 \sum_{k=1}^{N} \rho_{k}-\nu m^{2}\right)=\frac{1}{3}(N+1)(2 N+3)\left(\bar{\nu}_{N+1}-\nu\right) .
$$

А отсюда очевидным образом следует, что интересующее нас состояние равновесия $O_{N}$ экспоненциально устойчиво (неустойчиво) при $\nu-\bar{\nu}_{N+1}>0(<0)$.

Для завершения доказательства теоремы убедимся, что при условиях (1.24) аттрактор (1.22) не содержит устойчивых состояний равновесия, отличных от $O_{N}$. В связи с этим обратим внимание, что хотя $O_{m} \subset \widetilde{A}_{\nu}$ при $m=$ $1, \ldots, N-1$, но, как уже было отмечено выше, для рассматриваемых значений параметра $\nu$ все эти состояния равновесия неустойчивы. Тем самым, остается лишь проверить, что система (1.15) не допускает ни одного устойчивого состояния равновесия $O$ такого, что $O \in K$, но

$$
O \notin K_{m} \quad \forall m \geqslant 1 \text {. }
$$


Обратим внимание, что компоненты $\rho_{n}(t)$ каждого решения $\rho(t) \in K$ системы (1.15), номера $n$ которых удовлетворяют условию $1-\nu n^{2}<0$, в силу дифференциальных неравенств $\dot{\rho}_{n} \leqslant\left(1-\nu n^{2}\right) \rho_{n}$ стремятся к нулю при $t \rightarrow+\infty$. Поэтому, в частности, и любое ее состояние равновесия вида (1.32) (если оно существует) заведомо имеет лишь конечное число ненулевых координат.

Итак, фиксируем произвольно состояние равновесия $O$ из класса (1.32), компоненты которого согласно вышесказанному задаются равенствами

$$
\rho_{n_{j}}=\eta_{j}>0, \quad j=1, \ldots, k ; \quad \rho_{n}=0 \quad \forall n \in \mathbb{N} \backslash\left\{n_{j}\right\},
$$

где $n_{1}<n_{2}<\cdots<n_{k}$, а $k$ - некоторое конечное натуральное число. Подставляя, далее, соотношения (1.33) в (1.15), убеждаемся, что

$$
\eta_{j}=1-2 S_{*}-\nu n_{j}^{2}, \quad S_{*}=\frac{1}{2 k+1}\left(k-\nu \sum_{m=1}^{k} n_{m}^{2}\right), \quad j=1, \ldots, k,
$$

а требование $\min _{j} \eta_{j}=\eta_{k}>0$ эквивалентно условиям

$$
0 \leqslant \nu<\nu_{*}=\left[(2 k+1)\left(n_{k}^{2}-\frac{2}{2 k+1} \sum_{m=1}^{k} n_{m}^{2}\right)\right]^{-1} .
$$

Чтобы убедиться в неустойчивости состояния равновесия (1.33), (1.34), линеаризуем на нем исходную систему (1.15) и из получившейся системы в вариациях возьмем только одно уравнение для компоненты $h_{m}$ с номером $m$ таким, что $m<n_{k}, m \neq n_{j}, j=1, \ldots, k$. Подчеркнем, что в силу (1.32) такое $m$ обязательно найдется, а отвечающее ему уравнение имеет вид

$$
\dot{h}_{m}=\left(1-2 S_{*}-\nu m^{2}\right) h_{m}
$$

Остается добавить, что из условий (1.35) и неравенства $m<n_{k}$ вытекает строгая положительность фигурирующей в (1.36) величины $1-2 S_{*}-\nu m^{2}$. Теорема 1.1 полностью доказана.

Особый интерес представляет вопрос о структуре аттрактора $\widetilde{A}_{0}$, получающегося из (1.22) при $\nu=0$. Из проделанного выше анализа следует, что $\widetilde{A}_{0}$ содержит счетное число неустойчивых состояний равновесия вида

$$
\rho_{n_{j}}=\frac{1}{2 k+1}, \quad j=1, \ldots, k ; \quad \rho_{n}=0 \quad \forall n \in \mathbb{N} \backslash\left\{n_{j}\right\},
$$

где $n_{1}<n_{2}<\cdots<n_{k}-$ произвольный конечный набор натуральных чисел (в отличие от (1.33) здесь возможен и случай $n_{j}=j, j=1, \ldots, k$ ). Кроме того, неустойчивое многообразие любого из состояний равновесия (1.37) является бесконечномерным и также принадлежит множеству $\widetilde{A}_{0}$. А это означает, что аттрактор $\widetilde{A}_{0}$ заведомо некомпактен и имеет бесконечную хаусдорфову размерность, т. е. обладает свойствами, которые, по всей видимости, и должны быть присущи турбулентному аттрактору. Более детальная информация о его структуре содержится в следующем утверждении. 
Теорема 1.2. Для траектории $\rho(t)=\left(\rho_{1}(t), \ldots, \rho_{n}(t), \ldots\right)$ системы $(1.15)$ при $\nu=0$ с произвольным начальным условием $\rho(0)=\rho_{0} \in K, \rho_{0} \neq 0$, реализуется одна из следующих двух возможностей.

1) В случае, когда началъный вектор $\rho_{0}=\left(\rho_{1}^{0}, \ldots, \rho_{n}^{0}, \ldots\right)$ содержит бесконечное число ненулевых компонент, справедливы предельные равенства

$$
\lim _{t \rightarrow+\infty} \theta(t)=0, \quad \lim _{t \rightarrow+\infty} S(t)=1 / 2
$$

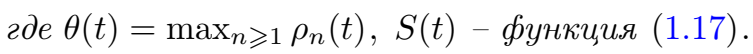

2) Если у вектора $\rho_{0}$ ненулевыми являются только координаты с номерами $n_{1}<n_{2}<\cdots<n_{k}$, где $k$ - некоторое конечное натуральное число, то

$$
\begin{aligned}
\lim _{t \rightarrow+\infty} \rho_{n_{j}}(t) & =\frac{1}{2 k+1}, \quad j=1, \ldots, k ; \\
\rho_{n}(t) & \equiv 0 \quad \forall n \in \mathbb{N} \backslash\left\{n_{j}\right\} .
\end{aligned}
$$

ДокАЗАТЕЛЬство. Рассмотрим сначала ситуацию, когда у начального вектора $\rho_{0}$ ненулевыми оказываются компоненты с номерами $n_{1}<\cdots<n_{j}<\cdots$ и таковых бесконечно много. В этом случае, очевидно, $\rho_{n}(t) \equiv 0$ при $n \neq n_{j}$, $j \geqslant 1$, а для $\eta_{j}(t)=\rho_{n_{j}}(t)$ получаем систему, которая с точностью до обозначений совпадает с исходной системой (1.15) при $\nu=0$. Поэтому без ограничения общности будем предполагать, что $\rho_{n}^{0}>0$ при всех $n \geqslant 1$.

Фиксируем произвольно номер $n$ и обратимся к уравнению для $\rho_{n}(t)$, имеющему вид

$$
\dot{\rho}_{n}=\left(1-2 S(t)-\rho_{n}\right) \rho_{n} .
$$

Далее, считая $S(t)$ в (1.40) уже известной функцией, найдем решение этого уравнения с начальным условием $\rho_{n}(0)=\rho_{n}^{0}>0$. На этом пути приходим к формулам

$$
\begin{gathered}
\rho_{n}(t)=\frac{\phi(t)}{\psi(t)} /\left(1+\frac{1}{\rho_{n}^{0} \psi(t)}\right), \\
\phi(t)=\exp \left\{\int_{0}^{t}(1-2 S(\tau)) d \tau\right\}, \quad \psi(t)=\int_{0}^{t} \phi(\sigma) d \sigma .
\end{gathered}
$$

Остановимся на некоторых необходимых для дальнейшего свойствах функций $\phi(t)$ и $\psi(t)$. А именно, покажем сначала, что

$$
\lim _{t \rightarrow+\infty} \psi(t)=+\infty
$$

С этой целью обратимся к оценке (1.19), из которой имеем

$$
1-2 S(t) \geqslant 1-2 S_{*}(t), \quad t \geqslant 0
$$

где $S_{*}(t)$ - решение задачи Коши (1.20), обладающее в случае $S_{0}=\sum_{n} \rho_{n}^{0}>0$ свойствами:

$$
\lim _{t \rightarrow+\infty} S_{*}(t)=\frac{1}{2}, \quad \int_{0}^{+\infty}\left|1-2 S_{*}(\tau)\right| d \tau<\infty .
$$


Учитывая, далее, факт сходимости фигурирующего в (1.44) интеграла, из неравенства (1.43) и из явного выражения для $\phi(t)($ см. (1.41)) последовательно выводим:

$$
\inf _{t \geqslant 0} \int_{0}^{t}(1-2 S(\tau)) d \tau>-\infty, \quad \inf _{t \geqslant 0} \phi(t)>0 .
$$

А отсюда и из формулы для $\psi(t)$ интересующее нас равенство (1.42) вытекает очевидным образом.

Еще одно свойство, которое нуждается в проверке, это ограниченность при $t \rightarrow+\infty$ функции $\phi(t) / \psi(t)$. Его справедливость следует из неравенства

$$
\frac{\phi(t)}{\psi(t)}=\rho_{n}(t)\left(1+\frac{1}{\rho_{n}^{0} \psi(t)}\right) \leqslant S_{*}(t)\left(1+\frac{1}{\rho_{n}^{0} \psi(t)}\right)
$$

и из уже установленного соотношения (1.42).

Приступим теперь непосредственно к доказательству теоремы и обоснуем сначала первое предельное равенство из (1.38). В связи с этим фиксируем произвольно натуральное $N$ и рассмотрим любой частичный предел $\gamma_{*} \geqslant 0$ отношения $\phi(t) / \psi(t)$ при $t \rightarrow+\infty$ (который в силу сказанного чуть выше заведомо конечен). Выберем, далее, последовательность $t_{k}>0, k \geqslant 1, t_{k} \rightarrow+\infty$, для которой $\phi\left(t_{k}\right) / \psi\left(t_{k}\right) \rightarrow \gamma_{*}$ при $k \rightarrow \infty$. Нетрудно увидеть (см. $\left.(1.41),(1.42)\right)$, что на этой последовательности

$$
\rho_{n}\left(t_{k}\right) \rightarrow \gamma_{*}, \quad k \rightarrow \infty, \quad n=1, \ldots, N .
$$

Но в то же время должно выполняться неравенство (см. (1.19))

$$
\sum_{n=1}^{N} \rho_{n}\left(t_{k}\right) \leqslant S_{*}\left(t_{k}\right)
$$

из которого при $k \rightarrow \infty$ с учетом свойств $(1.44),(1.45)$ выводим

$$
N \gamma_{*} \leqslant \frac{1}{2}
$$

А отсюда в силу произвольности $N$ с необходимостью имеем $\gamma_{*}=0$.

Следует отметить, что поскольку выше в качестве $\gamma_{*}$ брался любой частичный предел отношения $\phi(t) / \psi(t)$, то на самом деле в рассматриваемом случае

$$
\lim _{t \rightarrow+\infty} \frac{\phi(t)}{\psi(t)}=0 \text {. }
$$

Что же касается первого предельного соотношения (1.38), то оно вытекает из (1.46) и из очевидных оценок (см. (1.41))

$$
\rho_{n}(t)<\frac{\phi(t)}{\psi(t)} \quad \forall n \geqslant 1, \quad \forall t>0 .
$$

Для доказательства второго из свойств (1.38) попытаемся получить на $S(t)$ оценку снизу вида

$$
S_{* *}(t) \leqslant S(t), \quad t \in \mathbb{R}_{+},
$$


где $S_{* *}(t)$ - некоторая пока неизвестная функция. Для ее определения рассмотрим уравнение

$$
\dot{S}(t)=S(t)-2 S^{2}(t)-\sum_{n=1}^{\infty} \rho_{n}^{2}(t)
$$

которому в силу (1.15) удовлетворяет сумма $S(t)$, и учтем в нем очевидную оценку $\sum_{n} \rho_{n}^{2}(t) \leqslant \theta(t) S(t)$. В итоге приходим к дифференциальному неравенству

$$
\dot{S}(t) \geqslant(1-\theta(t)) S(t)-2 S^{2}(t),
$$

из которого, в свою очередь, следует, что в качестве функции $S_{* *}(t)$ в $(1.47)$ можно взять

$$
S_{* *}(t)=\frac{S_{0} \exp \left\{\int_{0}^{t}(1-\theta(\tau)) d \tau\right\}}{1+2 S_{0} \int_{0}^{t} \exp \left\{\int_{0}^{\tau}(1-\theta(\sigma)) d \sigma\right\} d \tau}, \quad S_{0}=\sum_{n=1}^{\infty} \rho_{n}^{0} .
$$

Проанализируем поведение при $t \rightarrow+\infty$ функции (1.49). Из уже доказанного свойства $\theta(t) \rightarrow 0, t \rightarrow+\infty$, очевидным образом выводим: при $t \rightarrow+\infty$

$$
\exp \left\{\int_{0}^{t}(1-\theta(\tau)) d \tau\right\} \rightarrow+\infty, \quad \int_{0}^{t} \exp \left\{\int_{0}^{\tau}(1-\theta(\sigma)) d \sigma\right\} d \tau \rightarrow+\infty .
$$

Поэтому в силу правила Лопиталя

$$
\lim _{t \rightarrow+\infty} S_{* *}(t)=\lim _{t \rightarrow+\infty} \frac{S_{0}(1-\theta(t))}{2 S_{0}}=\frac{1}{2} .
$$

А отсюда и из $(1.19),(1.44),(1.47)$ требуемое предельное равенство для $S(t)$ вытекает автоматически.

Перейдем теперь ко второму из указанных в теореме случаев и заметим, что, как и выше, тождества $\rho_{n}(t) \equiv 0$ при любом $n \in \mathbb{N} \backslash\left\{n_{j}\right\}$ очевидным образом следуют из структуры правых частей системы (1.15). Поэтому в доказательстве нуждаются только предельные соотношения из (1.39).

Покажем сначала, что в данном случае

$$
\lim _{t \rightarrow+\infty} S(t)=\frac{k}{2 k+1} .
$$

В связи с этим снова обратимся к уравнению (1.48), которое теперь принимает вид

$$
\dot{S}(t)=S(t)-2 S^{2}(t)-\sum_{j=1}^{k} \rho_{n_{j}}^{2}(t)
$$

и учтем в его правой части оценку

$$
\sum_{j=1}^{k} \rho_{n_{j}}^{2}(t) \geqslant \frac{1}{k} S^{2}(t)
$$


справедливую в силу выпуклости функции $f(x)=x^{2}, x \in \mathbb{R}$. В результате получаем дифференциальное неравенство

$$
\dot{S}(t) \leqslant S(t)-\left(2+\frac{1}{k}\right) S^{2}(t)
$$

и, соответственно, оценку

$$
S(t) \leqslant \bar{S}(t), \quad t \geqslant 0
$$

где $\bar{S}(t)$ - решение задачи Коши

$$
\dot{S}=S-\left(2+\frac{1}{k}\right) S^{2},\left.\quad S\right|_{t=0}=\sum_{j=1}^{k} \rho_{n_{j}}^{0}>0 .
$$

Для того чтобы установить на $S(t)$ аналогичную оценку снизу, рассмотрим вытекающую из $(1.40),(1.51)$ серию дифференциальных неравенств

$$
\dot{\rho}_{n_{j}} \geqslant\left(1-2 \bar{S}(t)-\rho_{n_{j}}\right) \rho_{n_{j}}, \quad j=1, \ldots, k .
$$

Заметим, далее, что каждое из них приводит к соответствующей оценке

$$
\rho_{n_{j}}(t) \geqslant \underline{\rho_{n_{j}}}(t), \quad t \geqslant 0,
$$

где $\rho_{n_{j}}(t)$ - функция, определяемая посредством аналогичных (1.41) равенств

$$
\begin{gathered}
\underline{\rho_{n_{j}}}(t)=\frac{\underline{\phi}(t)}{\underline{\psi}(t)} /\left(1+\frac{1}{\rho_{n_{j}}^{0} \underline{\psi}(t)}\right), \\
\underline{\phi}(t)=\exp \left\{\int_{0}^{t}(1-2 \bar{S}(\tau)) d \tau\right\}, \quad \underline{\psi}(t)=\int_{0}^{t} \underline{\phi}(\sigma) d \sigma .
\end{gathered}
$$

И наконец, полагая $\underline{S}(t)=\sum_{j=1}^{k} \underline{\rho_{n_{j}}}(t)$, приходим к выводу, что

$$
\underline{S}(t) \leqslant S(t), \quad t \geqslant 0 .
$$

Остановимся на характере поведения функций $\bar{S}(t), \underline{S}(t)$ при $t \rightarrow+\infty$. Для первой из них очевидным образом имеем

$$
\lim _{t \rightarrow+\infty} \bar{S}(t)=\frac{k}{2 k+1},
$$

так как она является решением задачи Коши (1.52). Далее, используя (1.55) в формулах для $\underline{\phi}(t)$ и $\underline{\psi}(t)$ из (1.53), убеждаемся, что

$$
\lim _{t \rightarrow+\infty} \underline{\phi}(t)=\lim _{t \rightarrow+\infty} \underline{\psi}(t)=+\infty .
$$

А отсюда, применяя правило Лопиталя, последовательно находим

$$
\begin{gathered}
\lim _{t \rightarrow+\infty} \underline{\rho_{n_{j}}}(t)=\lim _{t \rightarrow+\infty} \frac{\underline{\underline{\phi}}(t)}{\underline{\psi}(t)}=\lim _{t \rightarrow+\infty}(1-2 \bar{S}(t))=\frac{1}{2 k+1}, \quad j=1, \ldots, k ; \\
\lim _{t \rightarrow+\infty} \underline{S}(t)=\frac{k}{2 k+1} .
\end{gathered}
$$


И наконец, суммируя установленные факты (см. (1.51), (1.54)-(1.57)), получаем требуемое равенство (1.50).

Для завершения обоснования теоремы привлечем явные выражения для компонент $\rho_{n_{j}}(t), j=1, \ldots, k$. Подчеркнем, что они по-прежнему задаются формулами (1.41) (при $n=n_{j}$ ) и, в частности, фигурирующие в (1.41) функции $\phi(t)$ и $\psi(t)$ в силу уже установленного предельного равенства (1.50) обладают аналогичными (1.56) свойствами $\lim _{t \rightarrow+\infty} \phi(t)=\lim _{t \rightarrow+\infty} \psi(t)=+\infty$. Тем самым, снова применяя правило Лопиталя, приходим к выводу, что

$$
\lim _{t \rightarrow+\infty} \rho_{n_{j}}(t)=\lim _{t \rightarrow+\infty} \frac{\phi(t)}{\psi(t)}=\lim _{t \rightarrow+\infty}(1-2 S(t))=\frac{1}{2 k+1}, \quad j=1, \ldots, k .
$$

Теорема 1.2 полностью доказана.

Предельные равенства (1.38), реализующиеся для подавляющего большинства траекторий $\rho(t)$ системы (1.15) при $\nu=0$, на первый взгляд кажутся взаимоисключающими. Их одновременное выполнение приводит к следующему весьма парадоксальному свойству.

СледствиЕ. В первом из рассмотренных в теореме 1.2 случаев $\omega$-предельное множество любой траектории $\rho(t)$ является пустым.

Действительно, в предположении противного для некоторой траектории $\rho(t)$ найдется такая последовательность моментов времени $t_{k}, k \geqslant 1, t_{k} \rightarrow+\infty$ при $k \rightarrow+\infty$, что $\rho\left(t_{k}\right) \rightarrow \rho_{*}=\left(\rho_{1}^{*}, \ldots, \rho_{n}^{*}, \ldots\right) \in K$ по норме пространства $l$ (см. (1.16)). Далее, обратимся к серии очевидных неравенств

$$
\begin{gathered}
\max _{n \geqslant 1} \rho_{n}^{*} \leqslant \max _{n \geqslant 1} \rho_{n}\left(t_{k}\right)+\max _{n \geqslant 1}\left|\rho_{n}\left(t_{k}\right)-\rho_{n}^{*}\right| \leqslant \theta\left(t_{k}\right)+\left\|\rho\left(t_{k}\right)-\rho_{*}\right\|, \\
\left|\sum_{n=1}^{\infty} \rho_{n}^{*}-\frac{1}{2}\right| \leqslant\left|S\left(t_{k}\right)-\frac{1}{2}\right|+\left\|\rho\left(t_{k}\right)-\rho_{*}\right\|
\end{gathered}
$$

и перейдем в них к пределу при $k \rightarrow \infty$. В результате с учетом свойств $(1.38)$ и предполагаемого выше условия $\left\|\rho\left(t_{k}\right)-\rho_{*}\right\| \rightarrow 0, k \rightarrow \infty$, приходим к заключению, что одновременно $\max _{n} \geqslant 1 \rho_{n}^{*}=0$ и $\sum_{n} \rho_{n}^{*}=1 / 2$. Полученное противоречие доказывает пустоту $\omega$-предельного множества рассматриваемой траектории $\rho(t)$.

1.3. Итоговые выводы. Все результаты, установленные выше для нормальной формы, без труда переносятся на исходную краевую задачу (1.1). Действительно, обозначим через

$$
u(t, x)=\sum_{n=1}^{\infty}\left(z_{n}(t) \exp (i n x)+\bar{z}_{n}(t) \exp (-i n x)\right)
$$

произвольное ее решение с начальным условием

$$
u(x)=\sum_{n=1}^{\infty}\left(z_{n}^{0} \exp (i n x)+\bar{z}_{n}^{0} \exp (-i n x)\right) \in E_{0},
$$


заданным при $t=0$. Из доказанного в п. 1.2 факта нелокальной продолжимости траекторий $\rho(t) \in K$ системы (1.15) на полуось $t \geqslant 0$ и из соотношений $(1.13)$, связывающих $z_{n}(t)$ и $\rho_{n}(t), n \geqslant 1$, автоматически следует, что, во-первых, решение (1.58) также существует при всех $t \geqslant 0$; во-вторых, равенство

$$
G^{t}(u(x))=u(t, x), \quad t \in \mathbb{R}_{+},
$$

определяет в пространстве $E_{0}$ нелинейный полупоток, обладающий теми же общими свойствами, что и изученный в п. 1.2 полупоток (1.21).

В самом деле, проделанный выше анализ позволяет утверждать, что полупоток (1.60) при всех $\nu \geqslant 0$ имеет глобальный аттрактор $A_{\nu}$, связанный с аттрактором $\widetilde{A}_{\nu}$ полупотока (1.21) следующим образом: любой точке $\rho_{0}=$ $\left(\rho_{1}^{0}, \ldots, \rho_{n}^{0}, \ldots\right) \in \widetilde{A}_{\nu}$ отвечает семейство функций $u(x) \in A_{\nu}$, задающееся равенством (1.59), в котором $z_{n}^{0}=\sqrt{\rho_{n}^{0}} \exp \left(i \varphi_{n}^{0}\right), n \geqslant 1$, а вещественные параметры $\varphi_{n}^{0}, n \geqslant 1$, независимо друг от друга пробегают отрезок $[0,2 \pi]$.

Из отмеченного способа соответствия между точками множеств $\widetilde{A}_{\nu}, A_{\nu}$ и из теоремы 1.1 вытекает, что в случае, когда параметр $\nu$ при некотором натуральном $N$ удовлетворяет неравенствам (1.24), аттрактор $A_{\nu}$ содержит экспоненциально орбитально устойчивый $N$-мерный инвариантный тор

$$
T_{N}: u=\sum_{n=1}^{N} 2 \sqrt{\rho_{n}} \cos \left(n x+\varphi_{n}\right), \quad \dot{\varphi}_{n}=\sigma_{n}, \quad n=1, \ldots, N
$$

отвечающий состоянию равновесия $O_{N}$ системы (1.15). Здесь $\rho_{n}$ - амплитуды из (1.25), а частоты $\sigma_{n}$ заимствованы из (1.3). Добавим еще, что в силу условий, наложенных на последовательность $\sigma_{n}, n \geqslant 1$, при любом $N$ движения на торе (1.61) являются квазипериодическими.

При нарушении неравенств (1.24) тор (1.61) повторяет судьбу состояния равновесия $O_{N}$. А именно, при уменьшении параметра $\nu$ и при прохождении его через критическое значение $\nu=\bar{\nu}_{N+1}$ рассматриваемый тор теряет устойчивость, отдавая ее бифурцирующему из него инвариантному тору $T_{N+1}$. Таким образом, как и в краевой задаче (0.1), имеем бесконечную последовательность бифуркаций инвариантных торов

$$
T_{1} \rightarrow T_{2} \rightarrow \cdots \rightarrow T_{N} \rightarrow T_{N+1} \rightarrow \cdots
$$

с квазипериодической обмоткой на каждом из них. А это означает, что при $\nu \rightarrow 0$ в предложенной нами модификации примера Хопфа сохраняется сценарий перехода к турбулентности по Ландау.

Следует особо подчеркнуть, что главное преимущество нашего примера, из-за которого, собственно, он и был рассмотрен, состоит в том, что в отличие от задачи (0.1), где цепочка бифуркаций (1.62) не приводит ни к какому результату, в рамках краевой задачи (1.1) по прошествии всех этих бифуркаций, т. е. при $\nu=0$, возникает аттрактор $A_{0}$, который в дальнейшем будем называть турбулентным.

Суммируя проделанные построения, выделим четыре основных свойства турбулентного аттрактора. Сначала сформулируем три из них. 
1) Бесконечномерность. Хаусдорфова размерность множества $A_{0}$ заведомо бесконечна, так как оно содержит счетное число неустойчивых инвариантных торов сколь угодно высоких размерностей, отвечающих состояниям равновесия (1.37). Эти торы имеют вид

$$
T: u=\frac{2}{\sqrt{2 k+1}} \sum_{j=1}^{k} \cos \left(n_{j} x+\varphi_{j}\right), \quad \dot{\varphi}_{j}=\sigma_{n_{j}}, \quad j=1, \ldots, k,
$$

где $n_{1}<n_{2}<\cdots<n_{k}-$ произвольно фиксированный конечный набор натуральных чисел.

2) Некомпактность. Множество $A_{0}$ оказывается некомпактным (по тем же причинам, что и $\left.\widetilde{A}_{0}\right)$. Более того, для любого решения (1.58) краевой задачи (1.1) с начальным условием $u(0, x)=u(x) \in A_{0}$, содержащим бесконечное число ненулевых компонент $z_{n}^{0}$ (см. (1.59)), в силу теоремы 1.2 справедливы предельные равенства:

$$
\max _{n \geqslant 1}\left|z_{n}(t)\right| \rightarrow 0, \quad S(t)=\sum_{n=1}^{\infty}\left|z_{n}(t)\right|^{2} \rightarrow \frac{1}{2} \quad \text { при } t \rightarrow+\infty .
$$

Свойства (1.64), в свою очередь, гарантируют пустоту множества частичных пределов в пространстве $E_{0}$ у функции $u(t, x)$ при $t \rightarrow+\infty$.

3) ЖКесткая турбулентность. Термин “жесткая турбулентность" возник в связи с проблемой описания редких катастрофических событий в системах со сложным поведением, например, крупных ураганов и тайфунов в системе атмосфера-океан, экономических кризисов, различных крупномасштабных возмущений социальной среды, революций и т.д. Базовой математической моделью подобного рода явлений следует считать известное уравнение ГинзбургаЛандау

$$
w_{t}=\left(c_{1}+i c_{2}\right) \Delta w+w-\left(1+i c_{3}\right)|w|^{2} w
$$

$\left(w(t, x)\right.$ - комплекснозначная функция, $c_{1}>0, c_{2}, c_{3}$ - вещественные параметры любого знака), так как именно в нем в случае двух и трех пространственных переменных при больших $c_{2}, c_{3}$ была обнаружена жесткая турбулентность автоколебательный режим с редкими, но исключительно высокими выбросами на общем турбулентном фоне [15]. Впоследствии такого типа режим удалось выявить и в пространственно одномерном случае, но для уравнения, получающегося из (1.65) при замене $|w|^{2}$ на $|w|^{4}$ (см. [16]).

Один из возможных подходов к изучению жесткой турбулентности заключается в построении и анализе различных ее феноменологических моделей. Например, в монографии [17] в качестве соответствующей упрощенной модели бралось некоторое трехмерное отображение, а в статьях [18]-[20] исследовался иной класс моделей - сингулярно возмущенные системы обыкновенных дифференциальных уравнений с одной быстрой и $n(n \geqslant 3)$ медленными переменными. Что же касается введенной нами краевой задачи (1.1), то ее можно рассматривать как еще одну, но уже бесконечномерную феноменологическую модель явления жесткой турбулентности. 
Для пояснения сути дела рассмотрим принадлежащую тору (1.61) произвольную начальную функцию

$$
u_{N}\left(x, \nu, \varphi^{0}\right)=2 \sum_{n=1}^{N} \sqrt{\rho_{n}} \cos \left(n x+\varphi_{n}^{0}\right),
$$

где $\rho_{n}$ - амплитуды $(1.25), \nu \in\left(\bar{\nu}_{N+1}, \bar{\nu}_{N}\right), \varphi^{0}=\left(\varphi_{1}^{0}, \ldots, \varphi_{N}^{0}\right)-$ начальный фазовый вектор. Рассмотрим, далее, траекторию

$$
u_{N}\left(x, \nu, \varphi^{t}\right)=2 \sum_{n=1}^{N} \sqrt{\rho_{n}} \cos \left(n x+\varphi_{n}^{t}\right), \quad \varphi_{n}^{t}=\varphi_{n}^{0}+\sigma_{n} t, \quad t \geqslant 0,
$$

на торе $T_{N}$ с начальным условием (1.66). Как оказывается, при $t=0$ и при $\varphi_{n}^{0}=0, n=1, \ldots, N$, функция (1.67) имеет пик жесткой турбулентности. Связано это с тем, что последовательность $u_{N}(x, \nu, 0), N \geqslant 1$, является $\delta$-образной. Точнее говоря, справедливо следующее утверждение.

Теорема 1.3. Равномерно по $\nu \in\left(\bar{\nu}_{N+1}, \bar{\nu}_{N}\right)$ при $N \rightarrow \infty$ имеем

$$
\max _{-\pi \leqslant x \leqslant \pi}\left|u_{N}(x, \nu, 0)\right|=O(\sqrt{N}), \quad \frac{1}{2 \pi} \int_{0}^{2 \pi}\left|u_{N}(x, \nu, 0)\right|^{2} d x \leqslant \frac{1}{2} .
$$

Для доказательства первого свойства (1.68) достаточно обратиться к очевидному равенству

$$
\max _{-\pi \leqslant x \leqslant \pi}\left|u_{N}(x, \nu, 0)\right|=u_{N}(0, \nu, 0)=2 \sum_{n=1}^{N} \sqrt{\rho_{n}}
$$

и заметить, что

$$
\sum_{n=1}^{N} \sqrt{\rho_{n}} \leqslant N \sqrt{\rho_{1}}, \quad \sum_{n=1}^{N} \sqrt{\rho_{n}} \geqslant \sum_{n=1}^{[N / 2]} \sqrt{\rho_{n}} \geqslant \sum_{n=1}^{[N / 2]} \frac{1}{\sqrt{2 N+1}}=\frac{[N / 2]}{\sqrt{2 N+1}},
$$

так как в силу (1.25) $\max _{n} \rho_{n}=\rho_{1}$ и $\rho_{n} \geqslant 1 /(2 N+1)$ при $n \leqslant[N / 2]$ ([·] - целая часть). Второе же из этих свойств проверяется непосредственно:

$$
\begin{aligned}
& \frac{1}{2 \pi} \int_{0}^{2 \pi}\left|u_{N}(x, \nu, 0)\right|^{2} d x=2 \sum_{n=1}^{N} \rho_{n} \\
& \quad=\frac{2 N}{2 N+1}\left(1-\frac{\nu}{6}(N+1)(2 N+1)\right) \leqslant \frac{2 N}{2 N+1} \leqslant \frac{1}{2} .
\end{aligned}
$$

Наглядное представление о поведении функции $u_{N}(x, \nu, 0)$ при больших $N$ дает рис. 1.1, где изображен ее график на отрезке $-\pi \leqslant x \leqslant \pi$ при $N=$ $300, \nu=\left(\bar{\nu}_{N}+\bar{\nu}_{N+1}\right) / 2$. Из этого рисунка видно, что наблюдается картина, характерная для жесткой турбулентности, - асимптотически высокий всплеск на общем спокойном турбулентном фоне.

При последующем увеличении $t$ пик жесткой турбулентности, естественно, размывается (см. рис. 1.2, где приведен график функции (1.67) на отрезке 


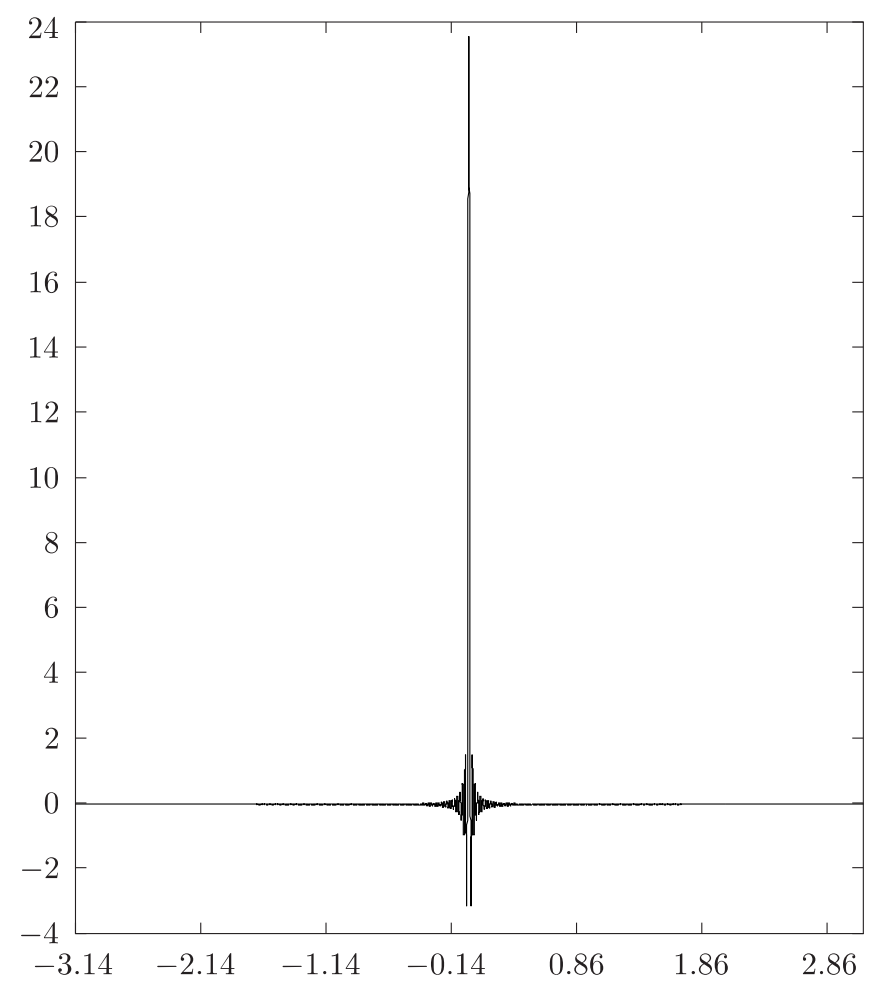

Рис. 1.1

$-\pi \leqslant x \leqslant \pi$ при $\left.N=300, t=1, \varphi_{n}^{0}=0, \sigma_{n}=n^{3}+\exp (-n), n=1, \ldots, N\right)$. Однако в силу квазипериодичности движений на торе (1.61) мы можем утверждать, что для любого начального фазового вектора $\varphi^{0}$ заведомо найдется такая последовательность моментов времени $t_{k}, k \geqslant 1, t_{k} \rightarrow \infty$ при $k \rightarrow \infty$, что функция $u_{N}\left(x, \nu, \varphi^{t_{k}}\right)$ будет иметь на отрезке $-\pi \leqslant x \leqslant \pi$ асимптотически высокий всплеск, аналогичный показанному на рис. 1.1.

Возвращаясь к турбулентному аттрактору $A_{0}$, заметим, что он содержит траектории, у которых с ростом $t$ возникают пики высоты, большей любого наперед заданного конечного значения. Таковыми являются, например, траектории, принадлежащие инвариантным торам (1.63).

Теперь сформулируем четвертое свойство турбулентного аттрактора.

4) Фрактальность. Описание данного свойства в общем случае возможно лишь на эвристическом уровне строгости, поскольку не существует универсального математического определения фрактальности. В нашей ситуации под фрактальностью будем понимать возникновение при $\nu \rightarrow 0$ пространственновременных структур сколь угодно малых масштабов по $t$ и $x$. Отметим, что ранее такого типа эффект был обнаружен в разностных уравнениях с непрерывным временем [21] и получил специальное название - сухая турбулентность. Следует также добавить, что в случае краевой задачи (1.1) речь, естественно, может идти только о динамических фракталах, для которых в отличие от раз- 


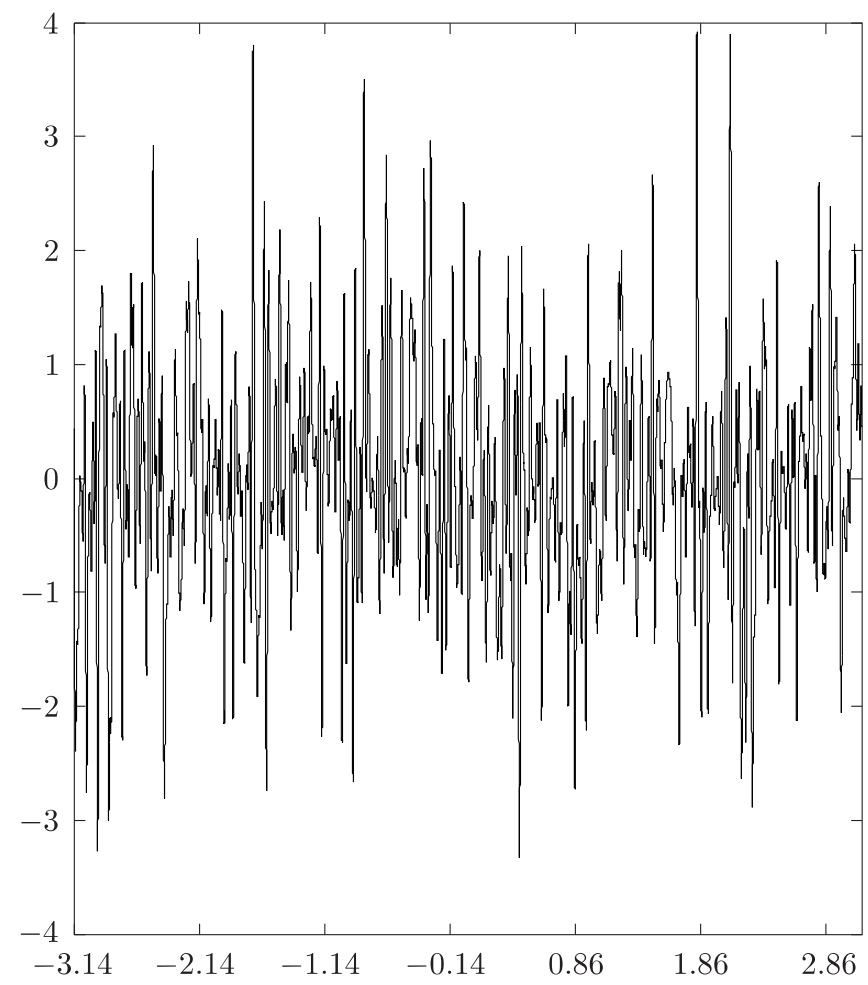

Рис. 1.2

личного рода конструктивных примеров фрактальных множеств (см. [22]) свойство масштабной инвариантности выполняется, вообще говоря, лишь приближенно.

Наличие фрактальности иллюстрируют рис. 1.3 и 1.4. На первом из этих рисунков изображен график функции (1.66) на отрезке $-\pi \leqslant x \leqslant \pi$ при $N=500$, $\nu=\left(\bar{\nu}_{N+1}+\bar{\nu}_{N}\right) / 2, \varphi_{n}^{0}=n^{3}+\exp (-n), n=1, \ldots, N$, а на втором - график функции (1.67) на промежутке $0 \leqslant t \leqslant 4$ при $x=0.1, \varphi_{n}^{0}=0, \sigma_{n}=n^{3}+\exp (-n)$, $n=1, \ldots, N$, и при тех же значениях $N, \nu$. Аналогичный вид имеют графики указанных функций и при других $N$.

Представленная визуальная информация, а также дополнительные численные расчеты свидетельствуют о том, что с ростом $N$ неограниченно увеличивается степень "изрезанности" траекторий (1.67) по переменным $t$ и $x$ одновременно. А это, собственно, и означает, что имеет место требуемое свойство фрактальности.

Подводя итог, отметим, что предпринятое в данном параграфе рассмотрение модельного примера (1.1) оказалось полезным по следующим двум причинам. Во-первых, как и в примере Хопфа, в нашем случае полностью прослежена вся счетная последовательность бифуркаций (1.62) и получены явные выражения как для критических значений параметра $\nu$, так и для возникающих инвариантных торов (см. (1.23), (1.61)). Во-вторых, и это самое главное, для 


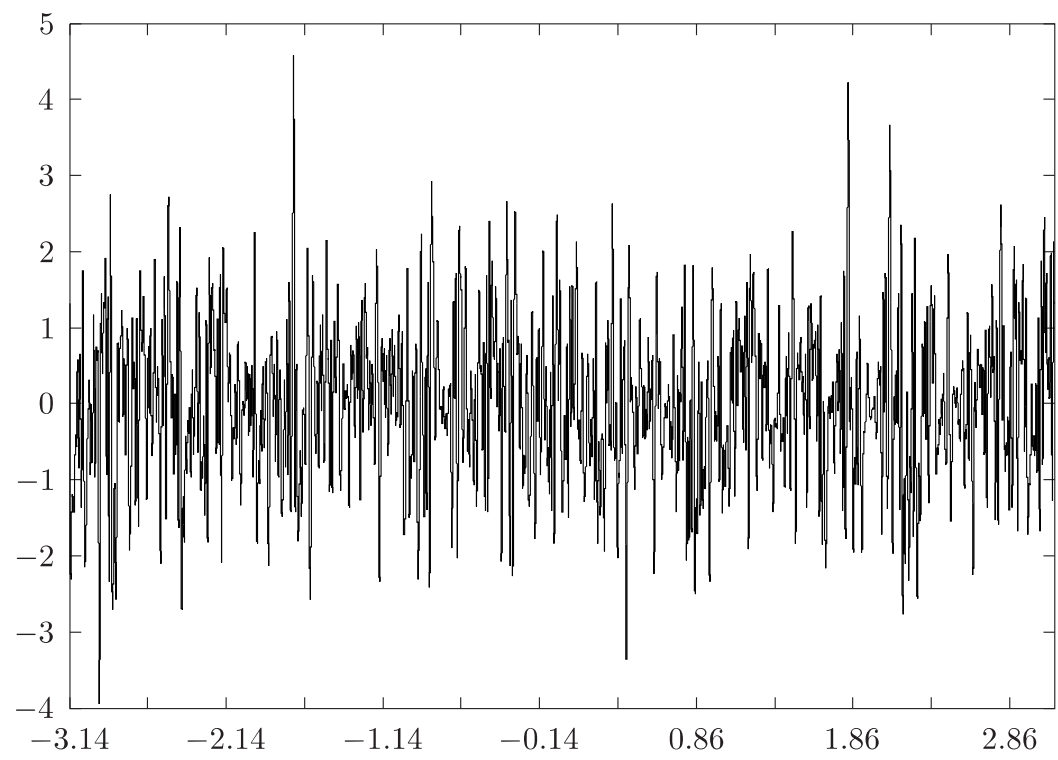

Рис. 1.3

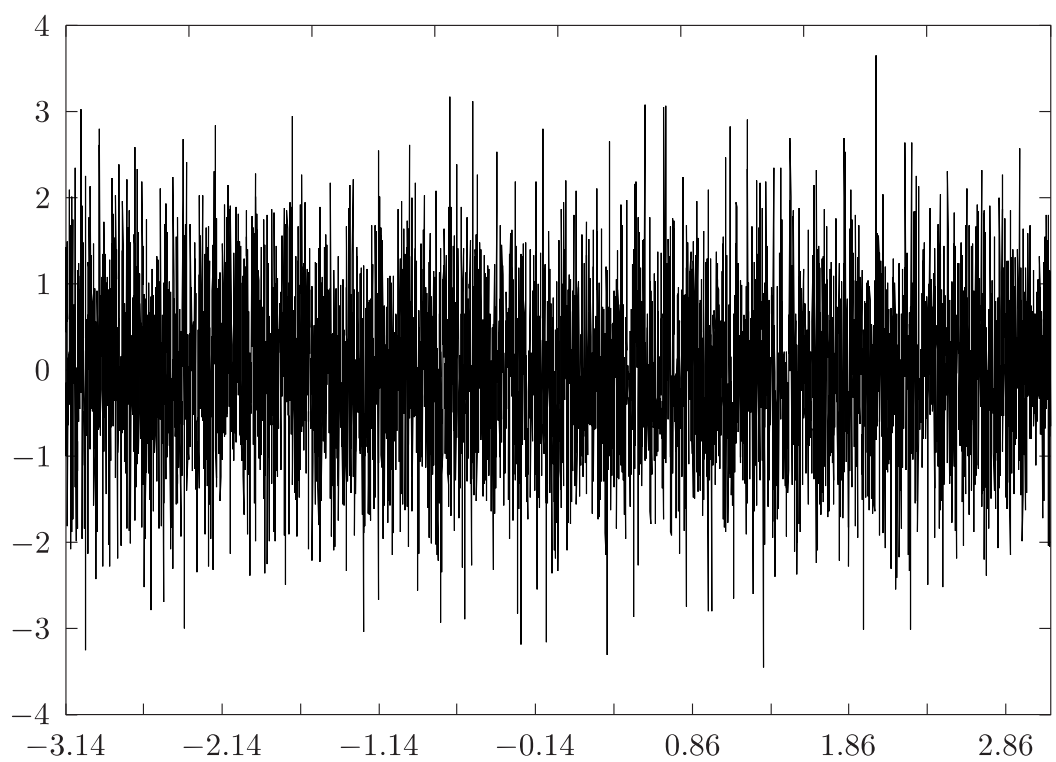

Рис. 1.4

краевой задачи (1.1) при $\nu=0$ удалось дать строгое математическое определение турбулентного аттрактора $A_{0}$ и выявить некоторые весьма тонкие его свойства. $\mathrm{K}$ сожалению, в более реалистичных физических примерах, которые рассматриваются ниже, исследовать проблему развития турбулентности с такой полнотой не представляется возможным. 


\section{§ 2. Турбулентные структуры на поверхности мелкой воды}

2.1. Разрешимость начально-краевой задачи. В настоящем параграфе изучается краевая задача вида

$$
u_{t}+u_{x x x}+\sqrt{\varepsilon} u u_{x}=\varepsilon \nu u_{x x}+\varepsilon u+\varepsilon g(u), \quad u(t, x+2 \pi) \equiv u(t, x) .
$$

Здесь $u(t, x)$ - вещественная скалярная функция, имеющая нулевое среднее значение по $x$, оператор $g$ действует по правилу

$$
g: u(x) \rightarrow-M\left(u^{2}\right) u(x)-u(x) * u(x) * u(-x),
$$

где, как и в (1.2), $M$ - среднее значение, *- свертка, а параметры $\varepsilon$ и $\nu$ таковы, что $0<\varepsilon \ll 1, \nu=$ const $>0$. С физической точки зрения эту задачу можно рассматривать как феноменологическую модель возникновения турбулентных структур на поверхности мелкой воды, поскольку уравнение из (2.1) является возмущением известного уравнения Кортевега-де Фриза (КдФ)

$$
u_{t}+u_{x x x}+u u_{x}=0 .
$$

Точнее говоря, оно получается из КдФ в результате нормировки $u / \sqrt{\varepsilon} \rightarrow u$ и последующего дописывания недостающих слагаемых.

Как будет установлено ниже, при согласованном стремлении к нулю параметров $\varepsilon$ и $\nu$ в краевой задаче (2.1) возникают устойчивые инвариантные торы сколь угодно высоких размерностей, т. е. происходит развитие турбулентности по Ландау. Более того, оказывается, что за ее динамику по параметру $\nu$ отвечает уже изученная модельная система (1.15). Однако в отличие от краевой задачи (1.1), которая в точности сводится к этой системе, здесь из-за наличия характерного для уравнения КдФ слагаемого $\sqrt{\varepsilon} u u_{x}$ такое сведение осуществляется лишь приближенно (с точностью до асимптотически малых по $\varepsilon$ слагаемых).

Причину, по которой краевые задачи (1.1) и (2.1) имеют одинаковые динамические свойства, отчасти проясняет следующее обстоятельство: при отбрасывании в (2.1) упомянутого выше слагаемого $\sqrt{\varepsilon} u u_{x}$ и при $\varepsilon=1$ приходим к задаче (1.1) с полем внешней силы, заданным равенством (1.3) в случае $\sigma_{n}=n^{3}$, $n \geqslant 1$.

Еще одна трудность, с которой сталкиваемся при исследовании краевой задачи (2.1), также связана с присутствием слагаемого $\sqrt{\varepsilon} u u_{x}$. А именно, усложняется проблема разрешимости для (2.1) смешанной задачи с начальным условием $u(0, x)=u_{0}(x)$ из подходящего функционального пространства. Один из возможных способов решения этой проблемы предлагается в настоящем пункте.

Наряду с введенным в п. 1.1 пространством $E_{0}$ в дальнейшем нам потребуются пространства $E_{k}, k \in \mathbb{N}$. Элементами $E_{k}$ являются вещественные $2 \pi$-периодические функции $u(x)$ класса $W_{2}^{k}[-\pi, \pi]$ с нулевым средним значением. Нормы в этих пространствах зададим посредством равенств

$$
\|u\|_{E_{k}}=\left(2 \sum_{n=1}^{\infty}(n+1)^{2 k}\left|z_{n}\right|^{2}\right)^{1 / 2}
$$

где, как и в (1.4), $z_{n}$ - коэффициенты разложения (1.5). 
Введенные выше пространства позволяют определить понятия классического и обобщенного решений краевой задачи (2.1). Для того чтобы сделать это, будем рассматривать $u(t, x)$ как абстрактную функцию $u(t)$ со значениями в $E_{1}$. Тогда и сама задача (2.1) записывается в виде следующего абстрактного дифференциального уравнения в пространстве $E_{1}$ :

$$
\dot{u}=L(\varepsilon) u+\sqrt{\varepsilon} \Psi(u, u)+\varepsilon g(u),
$$

где $L(\varepsilon)=-d^{3} / d x^{3}+\varepsilon \nu d^{2} / d x^{2}+\varepsilon: E_{1} \rightarrow E_{1}-$ замкнутый линейный оператор с плотной в $E_{1}$ областью определения $D_{L}=E_{4}$, а действующий из $E_{k+1} \times E_{k+1}$ в $E_{k}$ билинейный ограниченный оператор $\Psi$ определен равенством

$$
\Psi\left(u_{1}, u_{2}\right)=-\frac{1}{2} \frac{d}{d x}\left(u_{1}(x) u_{2}(x)\right) \quad \forall u_{1}(x), u_{2}(x) \in E_{k} .
$$

Классическим решением уравнения (2.4) на некотором отрезке $0 \leqslant t \leqslant \bar{t}$ назовем непрерывно дифференцируемую (в метрике пространства $E_{1}$ ) функцию $u(t)$ со значениями в $D_{L}$, удовлетворяющую при каждом $t \in[0, \bar{t}]$ этому уравнению. Обобщенным решением рассматриваемого уравнения с начальным условием $u(0)=u_{0} \in E_{1}$ будем называть непрерывное по $t \in[0, \bar{t}]$ решение интегрального уравнения

$$
u(t)=\exp (L(\varepsilon) t) u_{0}+\int_{0}^{t} \exp (L(\varepsilon)(t-\tau))[\sqrt{\varepsilon} \Psi(u(\tau), u(\tau))+\varepsilon g(u(\tau))] d \tau .
$$

Здесь $\exp (L(\varepsilon) t), t \geqslant 0,-$ аналитическая полугруппа линейных ограниченных операторов в пространстве $E_{1}$, определяемая на любой функции $u(x)$ вида $(1.5)$ с помощью аналогичного (1.10) равенства

$$
\begin{gathered}
\exp (L(\varepsilon) t) u=\exp (\varepsilon t) \sum_{n=1}^{\infty}\left(z_{n} \exp \left[i\left(n^{3} t+n x\right)-\varepsilon \nu n^{2} t\right]\right. \\
\left.+\bar{z}_{n} \exp \left[-i\left(n^{3} t+n x\right)-\varepsilon \nu n^{2} t\right]\right)
\end{gathered}
$$

В формулируемом ниже утверждении через $B_{r}$ обозначен замкнутый шар пространства $E_{1}$ радиуса $r>0$ с центром в нуле.

Теорема 2.1. Для любых фиксированнъх $\bar{t}, r>0$ существует такое $\varepsilon_{0}=$ $\varepsilon_{0}(\bar{t}, r)>0$, что при всех $0<\varepsilon \leqslant \varepsilon_{0}$ и при каждом $u_{0} \in B_{r}$ уравнение $(2.4)$ имеет на отрезке $0 \leqslant t \leqslant \bar{t}$ единственное обобщенное решение $u(t, \varepsilon)$ с начальнъм условием $u(0, \varepsilon)=u_{0}$. Кроме того, в случае $u_{0} \in B_{r} \cap D_{L}$ это решение оказывается классическим.

ДокАЗАТЕЛЬСтво. Основная идея обоснования заключается в построении корректной замены переменных, убивающей слагаемое порядка $\sqrt{\varepsilon}$ в правой части уравнения (2.4). Интересующую нас замену будем искать в виде

$$
u=v+\sqrt{\varepsilon} h_{2}(v, v),
$$

где $h_{2}(\cdot, \cdot): E_{k} \times E_{k} \rightarrow E_{k}-$ симметричный билинейный ограниченный оператор, удовлетворяющий уравнению

$$
L(\varepsilon) h_{2}(v, v)-h_{2}(L(\varepsilon) v, v)-h_{2}(v, L(\varepsilon) v)=v v_{x} .
$$


Вопрос о разрешимости уравнения (2.9) исследован в статье [23]. Как показано в этой работе, соответствующий анализ существенно упрощается при переходе к координатам Фурье. Действительно, для любых двух элементов

$$
\begin{gathered}
u=\sum_{n \in \mathbb{Z} \backslash\{0\}} u_{n} \exp (\text { inx }), \quad v=\sum_{n \in \mathbb{Z} \backslash\{0\}} v_{n} \exp (\text { inx }), \\
u_{-n}=\bar{u}_{n}, \quad v_{-n}=\bar{v}_{n}, \quad n \geqslant 1,
\end{gathered}
$$

из пространства $E_{k}$ имеем

$$
\begin{gathered}
L(\varepsilon) u=\sum_{n \in \mathbb{Z} \backslash\{0\}} \lambda_{n}(\varepsilon) u_{n} \exp (\text { inx }), \quad \lambda_{n}(\varepsilon)=i n^{3}-\varepsilon \nu n^{2}+\varepsilon ; \\
v v_{x}=\sum_{n \in \mathbb{Z} \backslash\{0\}} \frac{i n}{2}\left(\sum_{p+q=n} v_{p} v_{q}\right) \exp (i n x) ; \\
h_{2}(u, v)=\sum_{n \in \mathbb{Z} \backslash\{0\}} h_{n, 2} \exp (i n x), \quad h_{n, 2}=\sum_{p, q \in \mathbb{Z} \backslash\{0\}} h_{p, q}^{n, 2} u_{p} v_{q} .
\end{gathered}
$$

Далее, подставляя разложения (2.10)-(2.13) в (2.9), без труда убеждаемся, что для фигурирующих в (2.13) коэффициентов $h_{p, q}^{n, 2}$ выполнены равенства

$$
h_{p, q}^{n, 2}= \begin{cases}\frac{i n}{2}\left(\lambda_{n}-\lambda_{p}-\lambda_{q}\right)^{-1} & \text { при } p+q=n, \\ 0 & \text { при } p+q \neq n .\end{cases}
$$

Итак, установлено, что билинейный оператор, определяемый с помощью соотношений $(2.13),(2.14)$, является формальным решением уравнения (2.9). Однако возникает вопрос о корректности данного определения. Ответ на него содержится в следующем утверждении.

Лемма 2.1. Для каждого $k=0,1,2, \ldots$ оператор (2.13), (2.14) действует из $E_{k} \times E_{k}$ в $E_{k+1}$ и непрерьвен.

ДокАЗАТЕЛЬСтво. Прежде всего необходимо проверить, что $\lambda_{n}-\lambda_{p}-\lambda_{q} \neq 0$ в случае $p+q=n, p \neq 0, n$. Но это действительно так, поскольку

$$
\left|\lambda_{n}-\lambda_{p}-\lambda_{q}\right| \geqslant\left|n^{3}-p^{3}-q^{3}\right|=3|n p(p-n)| \geqslant \frac{3 n^{2}}{2} .
$$

Далее, введем в рассмотрение аналогичные (2.10) функции

$$
\tilde{u}=\sum_{n \in \mathbb{Z} \backslash\{0\}}\left|u_{n}\right| \exp (\text { inx }), \quad \tilde{v}=\sum_{n \in \mathbb{Z} \backslash\{0\}}\left|v_{n}\right| \exp (\text { inx })
$$

и заметим, что из способа задания нормы в пространстве $E_{k}$ (см. (2.3)) вытекают свойства

$$
\|\tilde{u}\|_{E_{k}}=\|u\|_{E_{k}}, \quad\|\tilde{v}\|_{E_{k}}=\|v\|_{E_{k}}, \quad\|u v\|_{E_{k}} \leqslant\|u\|_{E_{k}}\|v\|_{E_{k}} .
$$


С учетом этих фактов и уже установленной оценки (2.15) приходим к цепочке неравенств

$$
\begin{aligned}
\left\|h_{2}(u, v)\right\|_{E_{k+1}} & =\left(\sum_{n \in \mathbb{Z} \backslash\{0\}}(|n|+1)^{2 k+2}\left|\sum_{p+q=n} h_{p, q}^{n, 2} u_{p} v_{q}\right|^{2}\right)^{1 / 2} \\
& \leqslant \frac{1}{3}\left(\sum_{n \in \mathbb{Z} \backslash\{0\}}\left(1+\frac{1}{|n|}\right)^{2}(|n|+1)^{2 k}\left(\sum_{p+q=n}\left|u_{p}\right|\left|v_{q}\right|\right)^{2}\right)^{1 / 2} \\
& \leqslant \frac{2}{3}\|\tilde{u} \tilde{v}\|_{E_{k}} \leqslant \frac{2}{3}\|\tilde{u}\|_{E_{k}}\|\tilde{v}\|_{E_{k}}=\frac{2}{3}\|u\|_{E_{k}}\|v\|_{E_{k}} .
\end{aligned}
$$

Лемма 2.1 доказана.

Непосредственная проверка показывает, что построенная выше замена (2.8) приводит исходное уравнение (2.4) к требуемому виду

$$
\dot{v}=L(\varepsilon) v+\varepsilon H(v, \varepsilon),
$$

где

$$
\begin{aligned}
H=( & +\sqrt{\varepsilon} \Upsilon(v))^{-1}\left\{2 \Psi\left(v, h_{2}(v, v)\right)+\sqrt{\varepsilon} \Psi\left(h_{2}(v, v), h_{2}(v, v)\right)\right. \\
& \left.+g\left(v+\sqrt{\varepsilon} h_{2}(v, v)\right)\right\},
\end{aligned}
$$

$\Psi$ - билинейный оператор (2.5), а линейный ограниченный оператор $\Upsilon(v)$ действует из $E_{k}$ в $E_{k+1}$ при любом $k \geqslant 0$ по правилу

$$
\Upsilon(v) u=2 h_{2}(v, u) \quad \forall u \in E_{k} .
$$

Возвращаясь к доказательству теоремы 2.1, фиксируем произвольно элемент $u_{0} \in B_{r}$ и обозначим через $v_{0} \in E_{1}$ решение уравнения

$$
v+\sqrt{\varepsilon} h_{2}(v, v)=u_{0},
$$

которое в силу малости $\varepsilon$ и свойств оператора $h_{2}(v, v)$ (см. лемму 2.1) определяется однозначно. После этого дополним уравнение (2.16) начальным условием $\left.v\right|_{t=0}=v_{0}$ и перейдем к аналогичному (2.6) интегральному уравнению

$$
v(t)=\exp (L(\varepsilon) t) v_{0}+\varepsilon \int_{0}^{t} \exp (L(\varepsilon)(t-\tau)) H(v(\tau), \varepsilon) d \tau
$$

для нахождения соответствующего обобщенного решения.

Анализ получившегося уравнения требует некоторых дополнительных построений. В первую очередь фиксируем произвольно $\bar{t}>0$ и обозначим через $C=C\left([0, \bar{t}] ; E_{1}\right)$ банахово пространство непрерывных на отрезке $0 \leqslant t \leqslant \bar{t}$ вектор-функций $v(t) \in E_{1}$ с нормой

$$
\|v(t)\|_{C}=\max _{0 \leqslant t \leqslant \bar{t}}\|v(t)\|_{E_{1}} .
$$

Далее, введем в рассмотрение множество

$$
\Sigma_{0}=\left\{v(t) \in C:\left\|v(t)-\exp (L(\varepsilon) t) v_{0}\right\|_{C} \leqslant \bar{r}\right\},
$$


где постоянная $\bar{r}>0$ также произвольно фиксирована. И наконец, обозначим через $\Pi(v(t))$ оператор, порождаемый правой частью уравнения $(2.20)$.

Убедимся, что оператор П действует из $\Sigma_{0}$ в $\Sigma_{0}$ и является сжимающим. В связи с этим сначала проверим, что при любом $t>0$ линейный оператор $\exp (L(\varepsilon) t)$ переводит $E_{0}$ в $E_{1}$. Действительно, привлекая разложение $(2.7)$, приходим к выводу, что

$$
\begin{aligned}
& \exp (-\varepsilon t)\|\exp (L(\varepsilon) t) u\|_{E_{1}}=\left(2 \sum_{n=1}^{\infty}(1+n)^{2}\left|z_{n}\right|^{2} \exp \left(-2 \varepsilon \nu n^{2} t\right)\right)^{1 / 2} \\
& \quad=\frac{1}{\sqrt{\varepsilon \nu t}}\left(2 \sum_{n=1}^{\infty}\left(1+\frac{1}{n}\right)^{2}\left(n \sqrt{\varepsilon \nu t} \exp \left(-n^{2} \varepsilon \nu t\right)\right)^{2}\left|z_{n}\right|^{2}\right)^{1 / 2} \leqslant \frac{c_{*}}{\sqrt{\varepsilon \nu t}}\|u\|_{E_{0}}
\end{aligned}
$$

где $c_{*}=2 \max _{\sigma \geqslant 0}(\sqrt{\sigma} \exp (-\sigma))$.

Остановимся, далее, на некоторых свойствах фигурирующего в (2.16) оператора $H(\cdot, \varepsilon): E_{k+1} \rightarrow E_{k}$. Из формул $(2.17),(2.18)$ и из явного выражения для $g(u)$ (см. (2.2)) вытекает существование для любых целых $k \geqslant 0, m \geqslant 1$ и произвольного множества $\Omega \subset E_{k+1}$ такого $\bar{\varepsilon}=\bar{\varepsilon}(k, m, \Omega)>0$, что при всех $0 \leqslant \varepsilon \leqslant \bar{\varepsilon}$ рассматриваемый оператор действует из $\Omega$ в $E_{k}$, а его производные Фреше $H_{v}^{(s)}(v, \varepsilon), s=0,1, \ldots, m$, непрерывны и ограничены по совокупности переменных $(v, \varepsilon) \in \Omega \times[0, \bar{\varepsilon}]$.

Из перечисленных свойств оператора $H(v, \varepsilon)$ следует, в частности, существование универсальных (не зависящих от $\varepsilon$ ) постоянных $\Delta_{0}, \Delta_{1}>0$ таких, что

$$
\begin{gathered}
\|H(v(t), \varepsilon)\|_{E_{0}} \leqslant \Delta_{0}, \quad\left\|H_{v}^{\prime}(v(t), \varepsilon)\right\|_{E_{1} \rightarrow E_{0}} \leqslant \Delta_{1} \\
\forall v(t) \in \Sigma_{0}, \quad \forall t \in[0, \bar{t}] .
\end{gathered}
$$

Далее, объединяя (2.24) с неравенством (2.23), приходим к выводу, что

$$
\begin{aligned}
& \left\|\Pi(v(t))-\exp (L(\varepsilon) t) v_{0}\right\|_{E_{1}} \leqslant \varepsilon c_{*} \int_{0}^{t} \frac{\exp (\varepsilon(t-\tau))}{\sqrt{\varepsilon \nu(t-\tau)}}\|H(v(\tau), \varepsilon)\|_{E_{0}} d \tau \\
& \quad \leqslant \sqrt{\frac{\varepsilon}{\nu}} c_{*} \Delta_{0} \int_{0}^{t} \frac{\exp (\varepsilon s)}{\sqrt{s}} d s \quad \forall v(t) \in \Sigma_{0}, \\
& \left\|\Pi\left(v_{1}(t)\right)-\Pi\left(v_{2}(t)\right)\right\|_{E_{1}} \\
& \leqslant \varepsilon c_{*} \int_{0}^{t} \frac{\exp (\varepsilon(t-\tau))}{\sqrt{\varepsilon \nu(t-\tau)}}\left\|H\left(v_{1}(\tau), \varepsilon\right)-H\left(v_{2}(\tau), \varepsilon\right)\right\|_{E_{0}} d \tau \\
& \leqslant \sqrt{\frac{\varepsilon}{\nu}} c_{*} \Delta_{1} \int_{0}^{t} \frac{\exp (\varepsilon s)}{\sqrt{s}} d s \cdot\left\|v_{1}-v_{2}\right\|_{C} \quad \forall v_{1}(t), v_{2}(t) \in \Sigma_{0} .
\end{aligned}
$$

Неравенства (2.25), (2.26) свидетельствуют о том, что при подходящем уменьшении $\varepsilon$ интересующий нас оператор П переводит множество $(2.22)$ в себя и оказывается сжимающим (с константой сжатия порядка $\sqrt{\varepsilon / \nu}$ ). А отсюда и из принципа сжимающих отображений вытекает существование на отрезке $[0, \bar{t}]$ единственного обобщенного решения $v(t, \varepsilon), v(0, \varepsilon)=v_{0}$, уравнения $(2.16)$. 
На следующем этапе доказательства убедимся, что в случае $v_{0} \in D_{L}$ построенное выше решение $v(t, \varepsilon)$ является классическим. С этой целью введем в рассмотрение банахово пространство $C^{1}=C\left([0, \bar{t}] ; E_{4}\right) \cap C^{1}\left([0, \bar{t}] ; E_{1}\right)$ с нормой

$$
\|v(t)\|_{C^{1}}=\|v(t)\|_{C}+\|L(\varepsilon) v(t)\|_{C}+\|\dot{v}(t)\|_{C},
$$

где $\|\cdot\|_{C}-$ норма (2.21). Рассмотрим также аналогичное (2.22) множество

$$
\Sigma_{1}=\left\{v(t) \in C^{1}:\left\|v(t)-\exp (L(\varepsilon) t) v_{0}\right\|_{C^{1}} \leqslant \bar{r}\right\}
$$

где, как и ранее, постоянная $\bar{r}>0$ произвольно фиксирована. Заметим, что при условии $v_{0} \in D_{L}=E_{4}$ автоматически $\exp (L(\varepsilon) t) v_{0} \in C^{1}$, поскольку в этом случае

$$
\frac{d}{d t}\left(\exp (L(\varepsilon) t) v_{0}\right)=L(\varepsilon) \exp (L(\varepsilon) t) v_{0}=\exp (L(\varepsilon) t) L(\varepsilon) v_{0}
$$

Для проверки включения П $\Sigma_{1} \subset \Sigma_{1}$ и факта сжимаемости оператора П фиксируем произвольно функцию $v(t) \in \Sigma_{1}$ и положим $w(t)=\Pi(v(t))$. Из соотношений (2.20), (2.28) вытекает, что

$$
\begin{aligned}
L(\varepsilon) w(t) & =\exp (L(\varepsilon) t) L(\varepsilon) v_{0}+\varepsilon \int_{0}^{t} \exp (L(\varepsilon)(t-\tau)) L(\varepsilon) H(v(\tau), \varepsilon) d \tau \in C \\
\dot{w}(t)= & \exp (L(\varepsilon) t)\left[L(\varepsilon) v_{0}+\varepsilon H\left(v_{0}, \varepsilon\right)\right] \\
& +\varepsilon \int_{0}^{t} \exp (L(\varepsilon)(t-\tau)) H_{v}^{\prime}(v(\tau), \varepsilon) \dot{v}(\tau) d \tau \in C
\end{aligned}
$$

(при выводе второго из этих равенств проводилось интегрирование по частям).

Действительно, непрерывность функции (2.30) очевидна, а в случае функции (2.29) она устанавливается с использованием оценки (2.23) и вполне понятных свойств ограниченности и гладкости оператора $L(\varepsilon) H(\cdot, \varepsilon): E_{4} \rightarrow E_{0}$. Более того, из структуры правых частей равенств (2.29), (2.30) следует (см. аналогичное место выше), что оператор П заведомо обладает требуемыми свойствами, т. е. переводит множество (2.27) в себя и оказывается сжимающим. Тем самым, он имеет в $\Sigma_{1}$ единственную неподвижную точку $v(t, \varepsilon)$, которая служит одновременно и классическим решением уравнения (2.16).

Подводя итог, отметим, что все интересующие нас обобщенные решения исходного уравнения (2.4) с начальными условиями $u_{0} \in B_{r}$ задаются соотношением

$$
u(t, \varepsilon)=v(t, \varepsilon)+\sqrt{\varepsilon} h_{2}(v(t, \varepsilon), v(t, \varepsilon)),
$$

где $v(t, \varepsilon)$ - любое из построенных выше решений уравнения (2.16). Если же $u_{0} \in B_{r} \cap D_{L}$, то и начальное условие $v_{0}$, определяемое из уравнения $(2.19)$, будет принадлежать пространству $D_{L}$. Поэтому в данном случае классическими являются как решение $v(t, \varepsilon), v(0, \varepsilon)=v_{0}$, уравнения $(2.16)$, так и отвечающее ему решение (2.31). Теорема 2.1 доказана. 
2.2. Существование и устойчивость инвариантных торов. Для того чтобы разобраться с аттракторами уравнения (2.16), попытаемся его немного упростить. А именно, с помощью некоторой замены приведем его к нормальной форме Пуанкаре-Дюлака до кубических слагаемых включительно.

Построение требуемой замены осуществляется в несколько этапов. Сначала выявим ряд необходимых в последующем свойств оператора $H(v, \varepsilon)$. Из формул $(2.17),(2.18)$ очевидным образом следует, что он допускает представление

$$
H(v, \varepsilon)=H_{1}(v)+\sqrt{\varepsilon} H_{2}(v, \varepsilon),
$$

где $H_{1}: E_{k+1} \rightarrow E_{k}$ - ограниченная трилинейная форма, заданная равенством

$$
H_{1}(v)=2 \Psi\left(v, h_{2}(v, v)\right)+g(v),
$$

a оператор $H_{2}(\cdot, \varepsilon): E_{k+1} \rightarrow E_{k}$ обладает теми же общими свойствами ограниченности и гладкости, что и исходный оператор $H(v, \varepsilon)$. Кроме того, его тейлоровское разложение в точке $v=0$ начинается с членов четвертого порядка.

Далее нам потребуется координатное представление оператора $H_{1}(v)$. Для его получения подставим в (2.33) разложение Фурье функции $v$ (см. (2.10)). На этом пути с учетом соотношений $(2.13),(2.14)$ и (2.2) приходим к соотношениям вида

$$
H_{1}(v)=\sum_{n \in \mathbb{Z} \backslash\{0\}} f_{n} \exp (\text { inx }), \quad f_{n}=\sum_{p_{1}+p_{2}+p_{3}=n} f_{p_{1}, p_{2}, p_{3}}^{n} v_{p_{1}} v_{p_{2}} v_{p_{3}} .
$$

Равенства (2.34) позволяют выделить из $H_{1}(v)$ резонансное и нерезонансное слагаемые. Для того чтобы сделать это, запишем $f_{n}$ в виде $f_{n}^{1}+f_{n}^{2}$, где в случае $f_{n}^{2}$ суммирование ведется по всем индексам $p_{1}+p_{2}+p_{3}=n, p_{j} \neq n$, $j=1,2,3$. В результате имеем

$$
H_{1}(v)=H_{1}^{1}(v)+H_{1}^{2}(v), \quad H_{1}^{j}(v)=\sum_{n \in \mathbb{Z} \backslash\{0\}} f_{n}^{j} \exp (\text { inx }), \quad j=1,2 .
$$

Что же касается интересующей нас проблемы нормализации, то она заключается в нахождении замены переменной, убивающей нерезонансное слагаемое $\varepsilon H_{1}^{2}(v)$ в правой части уравнения (2.16).

Нормализующую замену будем искать в виде

$$
v=w+\varepsilon h_{3}(w, w, w),
$$

где $h_{3}(\cdot, \cdot, \cdot): E_{k} \times E_{k} \times E_{k} \rightarrow E_{k}-$ симметричный трилинейный ограниченный оператор, удовлетворяющий уравнению

$L(\varepsilon) h_{3}(w, w, w)-h_{3}(L(\varepsilon) w, w, w)-h_{3}(w, L(\varepsilon) w, w)-h_{3}(w, w, L(\varepsilon) w)=-H_{1}^{2}(w)$

и допускающий в координатах Фурье представление

$$
h_{3}(u, v, w)=\sum_{n \in \mathbb{Z} \backslash\{0\}} h_{n, 3} \exp (\text { inx }), \quad h_{n, 3}=\sum_{p_{1}, p_{2}, p_{3} \in \mathbb{Z} \backslash\{0\}} h_{p_{1}, p_{2}, p_{3}}^{n, 3} u_{p_{1}} v_{p_{2}} w_{p_{3}} .
$$


Здесь $u, v, w$ - произвольные элементы из $E_{k}$, а $u_{p_{1}}, v_{p_{2}}, w_{p_{3}}$ - соответствующие коэффициенты их рядов Фурье (см., например, (2.10)).

При анализе уравнения (2.37) существенным оказывается то обстоятельство, что в силу $(2.2),(2.13),(2.14),(2.33)$ компоненты $f_{n}^{2}$ нерезонансного слагаемого $H_{1}^{2}(w)$ из $(2.35)$ имеют вид

$$
f_{n}^{2}=-i n \sum_{\substack{p_{1}+p_{2}+p_{3}=n \\ p_{j} \neq 0, n}} h_{p_{2}, p_{3}}^{p_{2}+p_{3}, 2} w_{p_{1}} w_{p_{2}} w_{p_{3}}, \quad n \in \mathbb{Z} \backslash\{0\}
$$

Действительно, из соотношений (2.39) и из структуры самого уравнения (2.37) автоматически следует, что фигурирующие в (2.38) комплексные коэффициенты $h_{p_{1}, p_{2}, p_{3}}^{n, 3}$ должны задаваться равенствами

$$
h_{p_{1}, p_{2}, p_{3}}^{n, 3}=\left\{\begin{aligned}
i n h_{p_{2}, p_{3}}^{p_{2}+p_{3}, 2}\left[\lambda_{n}(\varepsilon)-\lambda_{p_{1}}(\varepsilon)-\lambda_{p_{2}}(\varepsilon)-\lambda_{p_{3}}(\varepsilon)\right]^{-1} \\
\text { при } p_{1}+p_{2}+p_{3}=n, p_{j} \neq 0, \quad n, j=1,2,3 \\
0 \quad \text { во всех остальных ситуациях. }
\end{aligned}\right.
$$

Но при этом, как и в случае уравнения (2.9), возникает проблема корректности построенного таким способом оператора $h_{3}(u, v, w)$. Решение указанной проблемы содержится в следующем утверждении.

Лемма 2.2. При каждом $k=0,1,2, \ldots$ бормулъ $(2.38),(2.40)$ задают ограниченный трилинейный оператор, действующий из $E_{k} \times E_{k} \times E_{k}$ в $E_{k}$.

ДоказАтельство. Как и при обосновании леммы 2.1, убедимся сначала, что отличны от нуля знаменатели $\lambda_{n}-\lambda_{p_{1}}-\lambda_{p_{2}}-\lambda_{p_{3}}$ в (2.40). Следуя [23], покажем, что при $p_{1}+p_{2}+p_{3}=n, p_{j} \neq 0, n, j=1,2,3$, справедливы оценки

$$
\left|\lambda_{n}-\sum_{j=1}^{3} \lambda_{p_{j}}\right| \geqslant\left|n^{3}-p_{1}^{3}-p_{2}^{3}-p_{3}^{3}\right|=3\left|p_{1}+p_{2}\right| \cdot\left|n-p_{1}\right| \cdot\left|n-p_{2}\right| \geqslant 3|n| .
$$

Точнее говоря, в проверке нуждается только второе из неравенств $(2.41)$ и только при $n \geqslant 2$ (при $|n|=1$ оно очевидно, а случай $n \leqslant-2$ сводится к указанному посредством замен $\left.p_{j} \rightarrow-p_{j}, j=1,2\right)$.

Предположим сначала, что $0<p_{1}+p_{2}<n / 2$. Если дополнительно $p_{1}, p_{2}>0$, то $p_{1}+p_{2} \geqslant 2,\left|n-p_{1}\right|>n / 2,\left|n-p_{2}\right|>n / 2$ и, следовательно, требуемая оценка выполняется. Если же $p_{1} p_{2}<0$, то либо $\left|n-p_{1}\right|>n$, либо $\left|n-p_{2}\right|>n$ и снова убеждаемся в справедливости нужного неравенства. Случай $-n / 2<p_{1}+p_{2}<0$ разбирается аналогично.

Предположим теперь, что выполняется неравенство $\left|p_{1}+p_{2}\right| \geqslant n / 2$. Если при этом $\left|n-p_{1}\right| \cdot\left|n-p_{2}\right| \geqslant 2$, то интересующая нас оценка доказана. Если же одновременно $\left|n-p_{1}\right|=\left|n-p_{2}\right|=1$, то требуемый факт по-прежнему имеет место, поскольку тогда $\left|p_{1}+p_{2}\right| \geqslant 2 n-2 \geqslant n$.

Объединяя установленные неравенства (2.41) с уже известной оценкой (2.15) и учитывая явные формулы (2.14), (2.40), приходим к выводу, что

$$
\left|h_{p_{1}, p_{2}, p_{3}}^{n, 3}\right| \leqslant \frac{1}{3}\left|h_{p_{2}, p_{3}}^{p_{2}+p_{3}, 2}\right| \leqslant \frac{1}{9\left|p_{2}+p_{3}\right|} \leqslant \frac{1}{9} .
$$


А отсюда очевидным образом имеем (см. аналогичное место в доказательстве леммы 2.1)

$$
\begin{aligned}
\left\|h_{3}(u, v, w)\right\|_{E_{k}} & =\left(\sum_{n \in \mathbb{Z} \backslash\{0\}}(|n|+1)^{2 k}\left|\sum_{p_{1}+p_{2}+p_{3}=n} h_{p_{1}, p_{2}, p_{3}}^{n, 3} u_{p_{1}} v_{p_{2}} w_{p_{3}}\right|^{2}\right)^{1 / 2} \\
& \leqslant \frac{1}{9}\|\tilde{u} \tilde{v} \widetilde{w}\|_{E_{k}} \leqslant \frac{1}{9}\|\tilde{u}\|_{E_{k}}\|\tilde{v}\|_{E_{k}}\|\widetilde{w}\|_{E_{k}}=\frac{1}{9}\|u\|_{E_{k}}\|v\|_{E_{k}}\|w\|_{E_{k}} .
\end{aligned}
$$

Лемма 2.2 доказана.

Из способа построения замены (2.36) следует, что результатом ее применения к уравнению (2.16) является уравнение вида

$$
\dot{w}=L(\varepsilon) w+\varepsilon H_{1}^{1}(w)+\varepsilon^{3 / 2} \widetilde{H}(w, \varepsilon),
$$

где, напомним, $H_{1}^{1}(w)$ - резонансное слагаемое из (2.35), а свойства оператора $\widetilde{H}(\cdot, \varepsilon): E_{k+1} \rightarrow E_{k}$ идентичны отмеченным выше свойствам оператора $H_{2}(v, \varepsilon)$ из (2.32). Далее, наряду с (2.42) введем в рассмотрение вспомогательное уравнение

$$
\dot{w}=L(\varepsilon) w+\varepsilon H_{1}^{1}(w),
$$

которое будем называть укороченной нормальной формой.

Как оказывается, инвариантные торы уравнения (2.43) могут быть найдены в явном виде. Для того чтобы убедиться в этом, перейдем в нем к координатной форме записи, полагая

$$
w=\sum_{n=1}^{\infty}\left(w_{n} \exp (i n x)+\bar{w}_{n} \exp (-i n x)\right) .
$$

В результате с учетом вытекающих из (2.13), (2.14), (2.33) формул

$$
\begin{gathered}
f_{n}^{1}=-w_{n} \sum_{k=1}^{\infty} d_{n, k}(\varepsilon)\left|w_{k}\right|^{2}, \\
d_{n, k}(\varepsilon)=2+2 i n\left(h_{n, k}^{n+k, 2}+h_{n,-k}^{n-k, 2}\right) \quad \text { при } n \neq k, \\
d_{n, n}(\varepsilon)=3+i n h_{n, n}^{2 n, 2}, \\
f_{-n}^{1}=\overline{f_{n}^{1}}, \quad n \geqslant 1,
\end{gathered}
$$

для компонент $w_{n}, n \geqslant 1$, из (2.44) получаем счетную систему обыкновенных дифференциальных уравнений

$$
\dot{w}_{n}=\left[\lambda_{n}(\varepsilon)-\varepsilon \sum_{k=1}^{\infty} d_{n, k}(\varepsilon)\left|w_{k}\right|^{2}\right] w_{n}, \quad n \geqslant 1 .
$$

В дальнейшем вместо укороченной нормальной формы (2.43) будем работать с эквивалентной ей системой

$$
\begin{aligned}
& \dot{\rho}_{n}=2 \varepsilon\left[1-\nu n^{2}-\sum_{k=1}^{\infty} \operatorname{Re}\left(d_{n, k}(\varepsilon)\right) \rho_{k}\right] \rho_{n}, \\
& \dot{\varphi}_{n}=n^{3}-\varepsilon \sum_{k=1}^{\infty} \operatorname{Im}\left(d_{n, k}(\varepsilon)\right) \rho_{k}, \quad n \geqslant 1,
\end{aligned}
$$


получающейся из (2.46) после выполнения аналогичных (1.13) замен

$$
w_{n}=\sqrt{\rho_{n}} \exp \left(i \varphi_{n}\right), \quad \rho_{n} \geqslant 0, \quad \varphi_{n} \in \mathbb{R}, \quad n \geqslant 1 .
$$

Точнее говоря, в первую очередь нам потребуется отщепляющаяся от $(2.47)$ система для $\rho_{n}, n \geqslant 1$, которая после замены $2 \varepsilon t \rightarrow t$ приобретает вид

$$
\dot{\rho}_{n}=\left[1-\nu n^{2}-\sum_{k=1}^{\infty} \operatorname{Re}\left(d_{n, k}(\varepsilon)\right) \rho_{k}\right] \rho_{n}, \quad n \geqslant 1,
$$

и которую следует рассматривать в пространстве $l_{1}$, состоящем из векторов

$$
\rho=\left(\rho_{1}, \ldots, \rho_{n}, \ldots\right):\|\rho\|=\sum_{n=1}^{\infty} n^{2}\left|\rho_{n}\right|<\infty .
$$

Именно она, как и система (1.15) в аналогичной ситуации, отвечает в конечном итоге за возможные аттракторы укороченной нормальной формы в фазовом пространстве $E_{1}$. Более того, при $\varepsilon=0$ система (2.49) переходит в (1.15), так как в силу (2.45), (2.14) имеем

$$
\operatorname{Re} d_{n, k}(0)=2 \quad \text { при } n \neq k, \quad \operatorname{Re} d_{n, n}(0)=3 .
$$

Равенства (2.50) позволяют воспользоваться результатами из 1 для того, чтобы найти состояния равновесия системы (2.49). Действительно, фиксируем произвольно натуральное $N$ и предположим, что параметр $\nu$ удовлетворяет условиям (1.24). Рассмотрим, далее, линейную алгебраическую систему

$$
1-\nu n^{2}=\sum_{k=1}^{N} \operatorname{Re}\left(d_{n, k}(\varepsilon)\right) \rho_{k}, \quad n=1, \ldots, N .
$$

Из проделанного в п. 1.2 анализа следует, что, во-первых, при указанных значениях $\nu$ и при всех достаточно малых $\varepsilon$ система (2.51) допускает единственное решение

$$
\rho_{n}=\rho_{n}(\varepsilon), \quad \rho_{n}(0)=\rho_{n, 0}, \quad n=1, \ldots, N,
$$

где $\rho_{n, 0}-$ координаты (1.25); во-вторых, решению (2.52) отвечает экспоненциально устойчивое (в метрике пространства $l_{1}$ ) состояние равновесия $O_{N}(\varepsilon)$ системы (2.49) с компонентами

$$
\rho_{n}=\rho_{n}(\varepsilon), \quad n=1, \ldots, N ; \quad \rho_{n}=0 \quad \text { при } n \geqslant N+1 .
$$

Возвращаясь к системе (2.43), заметим, что при условиях (1.24) на параметр $\nu$ аттрактором этой системы является экспоненциально орбитально устойчивый $N$-мерный инвариантный тор

$$
\begin{aligned}
& w=\sum_{k=1}^{N} 2 \sqrt{\rho_{k}(\varepsilon)} \cos \left(k x+\varphi_{k}\right), \\
\widetilde{T}_{N}(\varepsilon): & \\
\dot{\varphi}_{n} & =n^{3}-\varepsilon \sum_{k=1}^{N} \operatorname{Im}\left(d_{n, k}(\varepsilon)\right) \rho_{k}(\varepsilon), \quad n=1, \ldots, N,
\end{aligned}
$$


получающийся в результате подстановки соотношений (2.53) в формулы $(2.44)$, $(2.48)$ и в уравнения для $\varphi_{n}$ из (2.47). Далее, при переходе от укороченной нормальной формы к полной системе (2.42) тор (2.54) в силу его грубости, естественно, сохраняется и переходит в асимптотически близкий к нему тор $T_{N}(\varepsilon)$. Точнее говоря, справедливо следующее утверждение.

ТЕОРема 2.2. Пусть при некотором натуралъном $N$ параметр $\nu$ удовлетворяет условиям (1.24). Тогда для любого натурального $m$ можно указать такое достаточно малое $\varepsilon_{0}=\varepsilon_{0}(m, \nu, N)>0$, что при всех $0<\varepsilon \leqslant \varepsilon_{0}$ в фазовом пространстве $E_{1}$ уравнения (2.42) существует экспоненииально орбитально устойчивый $N$-мерныц инвариантный тор

$$
\begin{aligned}
T_{N}(\varepsilon): \quad w & =\sum_{k=1}^{N} 2 \sqrt{\rho_{k}(\varepsilon)} \cos \left(k x+\varphi_{k}\right)+\varepsilon^{3 / 2} W_{*}(\varphi, \varepsilon), \\
\dot{\varphi} & =\omega(\varepsilon)+\varepsilon^{3 / 2} \Phi_{*}(\varphi, \varepsilon) .
\end{aligned}
$$

Здесъ $\varphi=\operatorname{colon}\left(\varphi_{1}, \ldots, \varphi_{N}\right), \omega(\varepsilon)=\operatorname{colon}\left(\omega_{1}(\varepsilon), \ldots, \omega_{N}(\varepsilon)\right)$,

$$
\omega_{n}(\varepsilon)=n^{3}-\varepsilon \sum_{k=1}^{N} \operatorname{Im}\left(d_{n, k}(\varepsilon)\right) \rho_{k}(\varepsilon), \quad n=1, \ldots, N,
$$

а $2 \pi$-периодические по векторному аргументу $\varphi$ функции $W_{*} \in E_{1}, \Phi_{*} \in \mathbb{R}^{N}$ и их всевозможные частные производные по $\varphi$ до порядка $m$ включительно ограничены равномерно по $\varphi, \varepsilon$ в метрике пространств $E_{1} u \mathbb{R}^{N}$ соответственно.

Сформулированная теорема представляет собой частный случай установленного в [24; гл. 1] общего результата о бифуркации инвариантных торов в абстрактных нелинейных системах. Отметим, что в монографии [24] реализован геометрический подход к проблеме существования и устойчивости инвариантных торов, основанный на исследовании динамических свойств оператора сдвига по траекториям рассматриваемой системы за фиксированное конечное время $t_{0}>0$. Что же касается уравнения (2.42), то для него ввести такого рода оператор позволяет полученная выше теорема 2.1 .

2.3. Заключительные замечания. Из теоремы 2.2 следует, что в исходной краевой задаче (2.1) при согласованном стремлении к нулю параметров $\nu$ и $\varepsilon$ происходят перестройки вида (1.62), приводящие к последовательности устойчивых инвариантных торов $T_{k}, k \geqslant 1$. Однако динамика на них, вообще говоря, может и не быть квазипериодической. Кроме того, в отличие от модельного примера (1.1) здесь удается отследить хотя и сколь угодно большое, но конечное число таких бифуркаций. В первую очередь это связано с тем, что мы не можем допустить независимого изменения параметров $\varepsilon$ и $\nu$, так как, например, при обосновании теоремы 2.1 требуется малость величины $\sqrt{\varepsilon / \nu}$. По той же причине при $\nu=0, \varepsilon>0$ остаются открытыми как вопрос о разрешимости для (2.1) смешанной задачи с начальным условием $u(0, x)=u_{0}(x) \in E_{1}$, так и проблема существования турбулентного аттрактора. 
Перейдем к возможным обобщениям полученных результатов. Нетрудно заметить, что разработанная в пп. 2.1, 2.2 методика заведомо применима в случае, когда в качестве оператора $g(u)$ в $(2.1)$ взята произвольная кубическая нелинейность вида $g(u)=\tilde{g}(u, u, u)$, где $\tilde{g}(\cdot, \cdot, \cdot): E_{1} \times E_{1} \times E_{1} \rightarrow E_{0}$ - непрерывный симметричный трилинейный оператор, допускающий аналогичное (2.38) координатное представление

$$
\tilde{g}(u, v, w)=\sum_{n \in \mathbb{Z} \backslash\{0\}} g_{n} \exp (\text { inx }), \quad g_{n}=\sum_{p_{1}+p_{2}+p_{3}=n} g_{p_{1}, p_{2}, p_{3}}^{n} u_{p_{1}} v_{p_{2}} w_{p_{3}} .
$$

Существенным здесь является то обстоятельство, что в сумме для $g_{n}$ в $(2.56)$ присутствуют только мономы $u_{p_{1}} v_{p_{2}} w_{p_{3}}$ с номерами $p_{1}+p_{2}+p_{3}=n$. Данный факт гарантирует возможность приведения задачи (2.1) к нормальной форме вида (2.42), резонансное слагаемое $\varepsilon H_{1}^{1}(w)$ в которой по-прежнему имеет структуру

$$
H_{1}^{1}(w)=\sum_{n=1}^{\infty}\left(f_{n}^{1} \exp (i n x)+\overline{f_{n}^{1}} \exp (-i n x)\right), \quad f_{n}^{1}=-w_{n} \sum_{k=1}^{\infty} d_{n, k}(\varepsilon)\left|w_{k}\right|^{2} .
$$

Что же касается фигурирующих в $(2.57)$ коэффициентов $d_{n, k}(\varepsilon)$, то теперь они задаются аналогичными (2.45), но уже другими равенствами:

$$
\begin{aligned}
& d_{n, k}(\varepsilon)=-6 g_{n, k,-k}^{n}+2 i n\left(h_{n, k}^{n+k, 2}+h_{n,-k}^{n-k, 2}\right) \quad \text { при } n \neq k ; \\
& d_{n, n}(\varepsilon)=-3 g_{n, n,-n}^{n}+i n h_{n, n}^{2 n, 2} .
\end{aligned}
$$

Очевидно, что в случае произвольной нелинейности $g(u)$ из описанного выше класса цепочка бифуркаций (1.62) в краевой задаче $(2.1)$ при $\nu \rightarrow 0$, вообще говоря, не сохраняется. Однако остается в силе некоторый условный аналог теоремы 2.2 , справедливость которого также вытекает из общих утверждений монографии [24].

Для формулировки соответствующего результата рассмотрим систему

$$
\dot{\rho}_{n}=\left[1-\nu n^{2}-\sum_{k=1}^{\infty} a_{n k} \rho_{k}\right] \rho_{n}, \quad n \geqslant 1,
$$

где $a_{n k}=-6 \operatorname{Re} g_{n, k,-k}^{n}, n \neq k, a_{n n}=-3 \operatorname{Re} g_{n, n,-n}^{n}$, получающуюся из $(2.49)$ при учете равенств $(2.58)$ и при $\varepsilon=0$. Предположим, далее, что при некотором $\nu>0$ эта система допускает экспоненциально устойчивое или дихотомичное (в метрике пространства $l_{1}$ ) состояние равновесия $O$ с компонентами вида

$$
\rho_{n_{j}}=\eta_{j}^{0}>0, \quad j=1, \ldots, k ; \quad \rho_{n}=0 \quad \text { при любом } n \in \mathbb{N} \backslash\left\{n_{j}\right\},
$$

где $n_{1}<\cdots<n_{k}-$ произвольный конечный набор натуральных чисел. Тогда имеет место следующее утверждение.

ТЕОрема 2.3. Введенному выше состоянию равновесия (2.60) амплитудной системы (2.59) в исходной краевой задаче (2.1) при всех достаточно мальх $\varepsilon>0$ соответствует $k$-мерный инвариантный тор $T_{k}(\varepsilon)$ с теми же свойствами устойчивости. Указанный тор записывается в аналогичном (2.55) 
параметрическом виде:

$$
\begin{aligned}
T_{k}(\varepsilon): \quad u & =\sum_{j=1}^{k} 2 \sqrt{\rho_{j}(\varepsilon)} \cos \left(n_{j} x+\varphi_{j}\right)+\sqrt{\varepsilon} U_{*}(\varphi, \varepsilon), \\
\dot{\varphi} & =\omega(\varepsilon)+\varepsilon^{3 / 2} \Phi_{*}(\varphi, \varepsilon) .
\end{aligned}
$$

Здесъ $\varphi=\operatorname{colon}\left(\varphi_{1}, \ldots, \varphi_{k}\right), \omega(\varepsilon)=\operatorname{colon}\left(\omega_{1}(\varepsilon), \ldots, \omega_{k}(\varepsilon)\right)$,

$$
\omega_{j}(\varepsilon)=n_{j}^{3}-\varepsilon \sum_{s=1}^{k} \operatorname{Im}\left(d_{n_{j}, n_{s}}(\varepsilon)\right) \rho_{s}(\varepsilon), \quad j=1, \ldots, k,
$$

амплитуды $\rho_{j}(\varepsilon), \rho_{j}(0)=\eta_{j}^{0}$, определяются из системы

$$
1-\nu n_{j}^{2}=\sum_{s=1}^{k} \operatorname{Re}\left(d_{n_{j}, n_{s}}(\varepsilon)\right) \rho_{s}, \quad j=1, \ldots, k,
$$

a $2 \pi$-периодические по $\varphi$ вектор-функиии $U_{*}(\varphi, \varepsilon), \Phi_{*}(\varphi, \varepsilon)$ со значениями в $E_{1}$ $u \mathbb{R}^{k}$ соответственно обладают теми же свойствами ограниченности и гладкости, что и аналогичные функции $W_{*}, \Phi_{*}$ из (2.55).

Обратим внимание, что в качестве коэффициентов

$$
g_{p_{1}, p_{2}, p_{3}}^{n}, \quad g_{p_{1}, p_{2}, p_{3}}^{-n}=\bar{g}_{p_{1}, p_{2}, p_{3}}^{n}
$$

из (2.56) можно брать любую последовательность комплексных чисел, не меняющуюся при перестановках индексов $p_{1}, p_{2}, p_{3}$ таких, что $p_{1}+p_{2}+p_{3}=n$ (например, $g_{p_{1}, p_{3}, p_{2}}^{n}=g_{p_{1}, p_{2}, p_{3}}^{n}$ ), и допускающую оценку вида $\left|g_{p_{1}, p_{2}, p_{3}}^{n}\right| \leqslant c|n|$ с некоторой универсальной (не зависящей от $\left.n, p_{1}, p_{2}, p_{3}\right)$ постоянной $c>0$. Действительно, нетрудно проверить, что построенный по таким коэффициентам оператор $\tilde{g}$ будет обладать всеми требуемыми свойствами. А отсюда и из (2.58) следует, что за счет выбора нелинейности $g(u)$ из рассматриваемого класса можно реализовать в системе (2.59) любые значения коэффициентов $a_{n k}$. Единственное требование, которому должны удовлетворять эти коэффициенты, состоит в выполнении неравенства

$$
\sup _{n, k \in \mathbb{N}} \frac{\left|a_{n k}\right|}{n}<\infty .
$$

Простейшим примером нелинейности, для которой справедливы все перечисленные требования, служит $g(u)=M\left(u^{3}\right)-u^{3}$, где, напомним, $M(\cdot)-$ среднее значение по $x$. В этом случае, как нетрудно увидеть,

$$
a_{n n}=3 \quad \text { при } n \geqslant 1, \quad a_{n k}=6 \text { при } n \neq k,
$$

а значит, условие (2.62) автоматически выполняется. Что же касается системы (2.59) с коэффициентами (2.63), то при любом фиксированном $n \geqslant 1$ и при $\nu \in\left(1,1 /\left(2 n^{2}-1\right)\right)$ она имеет экспоненциально устойчивое состояние равновесия $O_{n}$ с компонентами

$$
\rho_{n}=\frac{\left(1-\nu n^{2}\right)}{3}, \quad \rho_{m}=0 \quad \text { при } m \neq n .
$$


Ясно также, что при $\nu \rightarrow 0$ количество сосуществующих состояний равновесия (2.64) неограниченно растет. Поэтому в силу теоремы 2.3 при указанном способе выбора $g(u)$ в краевой задаче (2.1) реализуется хорошо известное явление буферности (см., например, [25]).

Напомним, что о феномене буферности принято говорить, если в фазовом пространстве некоторой системы за счет подходящего выбора параметров можно добиться сосуществования любого наперед заданного конечного числа однотипных аттракторов (состояний равновесия, циклов, торов и т. д.). В рассмотренном выше случае буферность заведомо имеет место, так как при согласованном уменьшении параметров $\varepsilon$ и $\nu$ неограниченно растет количество сосуществующих устойчивых периодических движений задачи (2.1), отвечающих состояниям равновесия (2.64).

Несколько более сложная ситуация наблюдается, когда

$$
\begin{array}{ll}
a_{n n}=2 & \text { при } n \geqslant 1 ; \\
a_{n k}=1 & \text { при } n \neq k,|n-k| \leqslant m_{0} ; \\
a_{n k}=3 & \text { при } n \neq k,|n-k|>m_{0},
\end{array}
$$

где $m_{0}$ - некоторое фиксированное натуральное число. Непосредственная проверка показывает, что при условиях (2.65) и при $\nu=0$ система $(2.59)$ имеет счетное число экспоненциально устойчивых состояний равновесия $O_{n}, n \geqslant 1$, с координатами

$$
\begin{array}{rlrl}
\rho_{n+s} & =1 /\left(2+m_{0}\right) & \text { при } s=0,1, \ldots, m_{0} ; \\
\rho_{m}=0 & \text { при } m \in \mathbb{N} \backslash\left\{n+s: s=0,1, \ldots, m_{0}\right\} .
\end{array}
$$

А отсюда и из теоремы 2.3 заключаем, что в данном случае при $\varepsilon, \nu \rightarrow 0$ в фазовом пространстве $E_{1}$ краевой задачи (2.1) происходит неограниченное накапливание экспоненциально орбитально устойчивых $\left(m_{0}+1\right)$-мерных инвариантных торов.

Подводя итог, отметим, что аналоги теорем 2.1, 2.2 сохраняются для краевой задачи $(2.1),(2.2)$ и при отказе от условия $M(u)=0$. Для пояснения сути дела приведем в этом случае уравнение из (2.1) к более удобному для последующего анализа виду, полагая $u=u_{0}+u_{1}$, где $u_{0}=M(u), u_{1}=u-M(u)$. Далее, в получившейся системе для $u_{0}, u_{1}$ выполним замену $u_{1}=v_{0}+\sqrt{\varepsilon} h_{2}\left(v_{0}, v_{0}\right)$, где $h_{2}$ - оператор (2.13), (2.14). В результате приходим к системе

$$
\dot{u}_{0}=\varepsilon u_{0}+\varepsilon \mathscr{F}_{1}\left(u_{0}, v_{0}, \varepsilon\right), \quad \dot{v}_{0}=L(\varepsilon) v_{0}-\sqrt{\varepsilon} u_{0} \mathscr{D}_{0} v_{0}+\varepsilon \mathscr{F}_{2}\left(u_{0}, v_{0}, \varepsilon\right),
$$

где $\mathscr{D}_{0}=d / d x: E_{1} \rightarrow E_{0}$, а операторы

$$
\mathscr{F}_{1}(\cdot, \cdot, \varepsilon): \mathbb{R} \times E_{k} \rightarrow \mathbb{R}, \quad \mathscr{F}_{2}(\cdot, \cdot, \varepsilon): \mathbb{R} \times E_{k+1} \rightarrow E_{k}
$$

обладают теми же общими свойствами ограниченности и гладкости, что и оператор $H(v, \varepsilon)$ из $(2.16)$.

Проверим сначала, что в рассматриваемом случае справедлив аналог теоремы 2.1. В связи с этим дополним систему (2.66) произвольными начальными условиями

$$
\left.u_{0}\right|_{t=0}=u_{0}^{0} \in \mathbb{R},\left.\quad v_{0}\right|_{t=0}=v_{0}^{0} \in E_{1}
$$


и перейдем к эквивалентной системе интегральных уравнений

$$
\begin{aligned}
u_{0}(t)= & u_{0}^{0}+\varepsilon \Pi^{t}\left(u_{0}, v_{0}\right), \\
v_{0}(t)= & \exp (\widetilde{L}(\varepsilon) t) v_{0}^{0}+\varepsilon \int_{0}^{t} \exp (\widetilde{L}(\varepsilon)(t-\tau)) \\
& \times\left[\mathscr{F}_{2}\left(u_{0}^{0}+\varepsilon \Pi^{\tau}\left(u_{0}, v_{0}\right), v_{0}(\tau), \varepsilon\right)-\sqrt{\varepsilon} \Pi^{\tau}\left(u_{0}, v_{0}\right) \mathscr{D}_{0} v_{0}(\tau)\right] d \tau,
\end{aligned}
$$

где

$$
\Pi^{t}\left(u_{0}, v_{0}\right)=\int_{0}^{t}\left(u_{0}(s)+\mathscr{F}_{1}\left(u_{0}(s), v_{0}(s), \varepsilon\right)\right) d s, \quad \widetilde{L}(\varepsilon)=L(\varepsilon)-\sqrt{\varepsilon} u_{0}^{0} \mathscr{D}_{0} .
$$

Заметим, далее, что оператор $\widetilde{L}(\varepsilon)$, как и исходный оператор $L(\varepsilon)$, порождает в пространстве $E_{1}$ аналитическую полугруппу $\exp (\widetilde{L}(\varepsilon) t), t \geqslant 0$, для которой, в частности, сохраняется оценка (2.23). Поэтому анализ системы (2.68) идентичен описанному выше анализу интегрального уравнения (2.20), а значит, остаются в силе следующие факты. Во-первых, при подходящем уменьшении $\varepsilon$ задача Коши (2.66), (2.67) имеет единственное обобщенное решение на любом фиксированном отрезке $0 \leqslant t \leqslant \bar{t}$; во-вторых, в случае $v_{0}^{0} \in D_{L}$ это решение будет классическим.

Исследование аттракторов системы (2.66) сводится к аналогичному вопросу для ее укороченной нормальной формы. Проводя соответствующий подсчет (см. также аналогичные построения в [23]), убеждаемся, что здесь вместо (2.46) получается система вида

$$
\dot{w}_{n}=\left[\lambda_{n}(\varepsilon)-\sqrt{\varepsilon} i n w_{0}-\varepsilon \sum_{k=0}^{\infty} d_{n, k}(\varepsilon)\left|w_{k}\right|^{2}\right] w_{n}, \quad n \geqslant 0,
$$

где $w_{0} \in \mathbb{R}, w_{n} \in \mathbb{C}, n \geqslant 1$. Что же касается амплитудных переменных $\rho_{n}=$ $\left|w_{n}\right|^{2}, n \geqslant 0$, то для них после замены $2 \varepsilon t \rightarrow t$ и последующего предельного перехода при $\varepsilon \rightarrow 0$ имеем систему

$$
\begin{gathered}
\dot{\rho}_{0}=\left[1-2 \sum_{k=0}^{\infty} \rho_{k}\right] \rho_{0}, \\
\dot{\rho}_{n}=\left[1-\nu n^{2}-3 \rho_{n}-2 \sum_{\substack{k=1 \\
k \neq n}}^{\infty} \rho_{k}-\rho_{0}\right] \rho_{n}, \quad n \geqslant 1 .
\end{gathered}
$$

Введем в рассмотрение аналогичную (1.23) последовательность

$$
\bar{\nu}_{N}=\frac{6}{N(N+1)(4 N-1)}, \quad N \geqslant 1,
$$

критических значений параметра $\nu$. Непосредственная проверка показывает, что при всех $0<\nu<\bar{\nu}_{N}$ система (2.69) имеет состояние равновесия $O_{N}$ с координатами

$$
\begin{gathered}
\rho_{0}=\frac{1}{N+1}+\frac{\nu}{6} N(2 N+1), \quad \rho_{n}=\rho_{0}-\nu n^{2}, \quad n=1, \ldots, N ; \\
\rho_{n}=0 \quad \text { при } n \geqslant N+1 .
\end{gathered}
$$


Далее, нетрудно убедиться, что состояние равновесия (2.71) экспоненциально устойчиво при $\nu \in\left(\bar{\nu}_{N+1}, \bar{\nu}_{N}\right)$, а при уменьшении параметра $\nu$ и при прохождении его через критическое значение $\nu=\bar{\nu}_{N+1}$ (см. (2.70)) происходит бифуркация вида $O_{N} \rightarrow O_{N+1}$, т. е. устойчивым становится очередное состояние равновесия $O_{N+1}$. Таким образом, при $\nu \rightarrow 0$ имеем цепочку бифуркаций

$$
O_{1} \rightarrow \cdots \rightarrow O_{N} \rightarrow O_{N+1} \rightarrow \cdots
$$

которой в исходной краевой задаче (2.1) соответствует цепочка (1.62) бифуркаций устойчивых инвариантных торов. Точнее говоря, при подходящем уменьшении $\varepsilon$ мы можем гарантировать реализуемость любого конечного числа переходов вида $T_{k} \rightarrow T_{k+1}$.

\section{§ 3. Турбулентная экономическая динамика}

3.1. Вывод математической модели. Рассматриваемая ниже математическая модель экономических процессов базируется на идеях, изложенных в монографиях [26], [27]. Следуя [26], обозначим через $Y(t)$ национальный доход, а через $I(t)$ - индуцированные инвестиции. Скорости изменения этих величин определяются из системы дифференциальных уравнений

$$
\dot{Y}=I-a Y, \quad \dot{I}=b \dot{Y}-I, \quad a, b=\text { const }>0,
$$

которая известна в литературе, посвященной математическому моделированию макроэкономических процессов, под названием мультипликатор-акселератор (см., например, [26], [27]). Далее, согласно соображениям Дж. Р. Хикса [28] о "нижнем пороге" сокращения капиталовложений дополним модель (3.1) нелинейным слагаемым

$$
F=-c(\dot{Y})^{3}, \quad c=\text { const }>0,
$$

а точнее говоря, перейдем к системе

$$
\dot{Y}=I-a Y, \quad \dot{I}=b \dot{Y}-I+F .
$$

После этого исключим стандартным образом из (3.3) переменную $I$. В итоге получается нелинейное дифференциальное уравнение второго порядка

$$
\ddot{Y}+(a+1-b) \dot{Y}+a Y=F,
$$

которое уместно назвать точечной экономической моделью.

На следующем этапе перейдем от (3.4) к соответствующей распределенной модели. В связи с этим фиксируем некоторый экономический регион, представляющий собой ограниченную область $\Omega \subset \mathbb{R}^{2}$ с кусочно гладкой границей $\partial \Omega$, и попытаемся учесть, например, такие факторы, как межрегиональная торговля и неравномерное экономическое развитие различных районов области $\Omega$. Тем самым, теперь $Y=Y(t, x, y), I=I(t, x, y),(x, y) \in \Omega$. Пусть также $T=T(t, x, y)$ - активное торговое сальдо. Согласно [26], в пространственно неоднородном случае вместо (3.3) имеем систему

$$
\dot{Y}=I-a Y+T+d_{1} \Delta Y, \quad \dot{I}=b \dot{Y}-I+F, \quad \dot{T}=d_{2} \Delta Y-T,
$$


где точка означает частную производную по $t, \Delta$ - оператор Лапласа, $d_{1}, d_{2}-$ положительные постоянные.

Обращаем внимание, что в [26] система (3.5) рассматривалась только при $d_{1}=0$. Это означает, что учитывалось лишь межрегиональное взаимодействие, происходящее за счет торговли. В то же время ясно, что существуют и другие формы такого взаимодействия. K ним можно отнести, например, дотации из одного региона в другой. За это и отвечает слагаемое $d_{1} \Delta Y$ в первом уравнении из (3.5). Кроме того, будем рассматривать следующую модификацию нелинейности (3.2):

$$
F=-c \dot{Y} \iint_{\Omega}(\dot{Y})^{2} d x d y, \quad c=\text { const }>0 .
$$

Смысл перехода к новой нелинейности (3.6) состоит в том, что теперь замедление инвестиций зависит не только от $\dot{Y}$, но и от интегрального роста доходов во всем регионе $\Omega$.

Для удобства последующего анализа исключим из системы (3.5) переменные $I, T$. С этой целью продифференцируем по $t$ первое уравнение из (3.5) и в правой части получившегося равенства заменим $\dot{I}, \dot{T}$ правыми частями двух других уравнений данной системы. В итоге убеждаемся, что

$$
\ddot{Y}=(b-a) \dot{Y}+d_{1} \Delta \dot{Y}+d_{2} \Delta Y+F-(I+T) .
$$

Подставляя, далее, в (3.7) формулу (3.6), а также вытекающее из первого уравнения (3.5) соотношение $I+T=\dot{Y}+a Y-d_{1} \Delta Y$, приходим к выводу, что $Y$ является решением уравнения

$$
\ddot{Y}+(a+1-b) \dot{Y}+a Y-d_{1} \Delta \dot{Y}=\left(d_{1}+d_{2}\right) \Delta Y-c \dot{Y} \iint_{\Omega}(\dot{Y})^{2} d x d y .
$$

Получившееся уравнение необходимо дополнить каким-либо экономически осмысленным граничным условием. В качестве такового ниже будем рассматривать равенство

$$
\left.Y\right|_{\partial \Omega}=0
$$

означающее постоянство дохода на границе области $\Omega$, или соотношение

$$
\left.\frac{\partial Y}{\partial \vec{n}}\right|_{\partial \Omega}=0, \quad \vec{n}-\text { внешняя нормаль }
$$

характеризующее замкнутость экономического региона (отсутствие утечки доходов через границу области).

Обратимся сначала к краевой задаче (3.8), (3.9) и выполним в ней замену времени $\sqrt{a} t \rightarrow t$. Предполагая, далее, что

$$
\begin{gathered}
\frac{b-a-1}{\sqrt{a}}=\varepsilon, \quad 0<\varepsilon \ll 1 ; \quad \frac{d_{1}}{\sqrt{a}}=\varepsilon \nu, \quad \nu=\text { const } \geqslant 0 ; \\
\sigma=\sqrt{\frac{d_{1}+d_{2}}{a}} \sim 1,
\end{gathered}
$$


для функции $u=a^{1 / 4} c^{1 / 2} Y / \sqrt{\varepsilon}$ приходим к задаче

$$
u_{t t}-\varepsilon u_{t}-\varepsilon \nu \frac{\partial}{\partial t} \Delta u+u=\sigma^{2} \Delta u-\varepsilon u_{t} \cdot \iint_{\Omega}\left(u_{t}\right)^{2} d x d y,\left.\quad u\right|_{\partial \Omega}=0,
$$

которая и является основным объектом исследования в настоящем параграфе.

3.2. Общие свойства рассматриваемой краевой задачи. В качестве фазового пространства краевой задачи (3.12) возьмем $\left(u, u_{t}\right) \in \stackrel{\circ}{W} \underset{2}{1}(\Omega) \times L_{2}(\Omega)$, где, как обычно, через $\stackrel{\circ}{W} \underset{2}{1}(\Omega)$ обозначено замыкание в метрике пространства $W_{2}^{1}(\Omega)$ линеала гладких функций, удовлетворяющих условию $\left.u\right|_{\partial \Omega}=0$. Для того чтобы определить понятие обобщенного решения задачи (3.12) с начальными условиями из указанного пространства, положим $w_{1}=\mathscr{D} u, w_{2}=u_{t}$, где $\mathscr{D}: \stackrel{\circ}{W} \underset{2}{1}(\Omega) \rightarrow L_{2}(\Omega)$ - арифметический квадратный корень из оператора $I-\sigma^{2} \Delta, I-$ единичный оператор. В итоге в пространстве $E=L_{2}\left(\Omega ; \mathbb{R}^{2}\right)$, состоящем из вектор-функций

$$
w=\operatorname{colon}\left(w_{1}, w_{2}\right): w_{1}, w_{2} \in L_{2}(\Omega), \quad\|w\|_{E}=\left(\iint_{\Omega}\left(w_{1}^{2}+w_{2}^{2}\right) d x d y\right)^{1 / 2},
$$

получаем абстрактное дифференциальное уравнение вида

$$
\dot{w}=L(\varepsilon) w+\varepsilon F(w),
$$

где $L(\varepsilon)=\mathscr{V}_{0} \mathscr{D}-\varepsilon \mathscr{V}_{1} \mathscr{D}^{2}+\varepsilon \mathscr{V}_{2}$,

$$
\begin{gathered}
\mathscr{V}_{0}=\left(\begin{array}{cc}
0 & 1 \\
-1 & 0
\end{array}\right), \quad \mathscr{V}_{1}=\left(\begin{array}{cc}
0 & 0 \\
0 & \nu / \sigma^{2}
\end{array}\right), \quad \mathscr{V}_{2}=\left(\begin{array}{cc}
0 & 0 \\
0 & 1+\nu / \sigma^{2}
\end{array}\right), \\
F(w)=\operatorname{colon}\left(0,-w_{2} \iint_{\Omega}\left(w_{2}\right)^{2} d x d y\right) .
\end{gathered}
$$

Убедимся, далее, что оператор $L(\varepsilon)$ порождает в $E$ сильно непрерывную по $t \geqslant 0$ полугруппу линейных ограниченных операторов $\exp (L(\varepsilon) t)$. Для этого обозначим через $0<\lambda_{1} \leqslant \lambda_{2} \leqslant \cdots \leqslant \lambda_{k} \leqslant \cdots$ - собственные значения оператора $-\Delta$ с нулевым граничным условием Дирихле, а через $e_{k}(x, y)$, $\iint_{\Omega} e_{k}^{2}(x, y) d x d y=1, k \geqslant 1,-$ отвечающую им систему собственных функций. Из очевидных равенств

$$
\mathscr{D} w=\sum_{k=1}^{\infty} \omega_{k} w_{k} e_{k}(x, y), \quad \omega_{k}=\sqrt{1+\sigma^{2} \lambda_{k}}, \quad k \geqslant 1,
$$

где

$$
w(x, y)=\sum_{k=1}^{\infty} w_{k} e_{k}(x, y), \quad w_{k}=\iint_{\Omega} w(x, y) e_{k}(x, y) d x d y, \quad k \geqslant 1,
$$

и из явного выражения для $L(\varepsilon)$ вытекает, что интересующая нас полугруппа действует на любой элемент $w \in E$ по правилу

$$
\exp (L(\varepsilon) t) w=\sum_{k=1}^{\infty} \exp \left[\left(\omega_{k} \mathscr{V}_{0}-\varepsilon \omega_{k}^{2} \mathscr{V}_{1}+\varepsilon \mathscr{V}_{2}\right) t\right] w_{k} e_{k}(x, y)
$$


Полученная формула (3.16) позволяет ввести понятие обобщенного решения уравнения (3.13) тем же способом, как это было сделано в п. 2.1. А именно, в данном случае под обобщенным решением с начальным условием $w(0)=$ $w_{0} \in E$ будем понимать непрерывную вектор-функцию $w(t)$ со значениями в $E$, удовлетворяющую на некотором отрезке $0 \leqslant t \leqslant \bar{t}$ интегральному уравнению

$$
w(t)=\exp (L(\varepsilon) t) w_{0}+\varepsilon \int_{0}^{t} \exp (L(\varepsilon)(t-\tau)) F(w(\tau)) d \tau .
$$

Аналогом теоремы 2.1 здесь является следующее утверждение, справедливое в отличие от предыдущего случая и при $\nu=0$.

ТеОрема 3.1. По любым фиксированным $\bar{t}, r>0$ можно указать такое $\varepsilon_{0}=\varepsilon_{0}(\bar{t}, r)>0$, что при $0<\varepsilon \leqslant \varepsilon_{0}$ u nрu $w_{0} \in B_{r}=\left\{w \in E:\|w\|_{E} \leqslant r\right\}$ уравнение (3.13) имеет на отрезке $0 \leqslant t \leqslant \bar{t}$ единственное обобщенное решение $w=w(t, \varepsilon), w(0, \varepsilon)=w_{0}$.

Доказательство сформулированной теоремы базируется на следующих двух фактах. Во-первых, из разложения (3.16) и из явного вида матриц $\mathscr{V} j, j=0,1,2$ (см. (3.14)), заключаем, что

$$
\|\exp (L(\varepsilon) t)\|_{E \rightarrow E} \leqslant c_{1} \exp \left(\varepsilon c_{2} t\right) \quad \forall t \geqslant 0,
$$

где $c_{1}, c_{2}>0$ - некоторые универсальные (не зависящие от $\varepsilon, t$ ) постоянные. Во-вторых, оператор $F(w)$ из (3.14) представляет собой непрерывную кубическую форму, действующую из $E$ в $E$.

Действительно, опираясь на перечисленные свойства, нетрудно убедиться (см. соответствующее место в доказательстве теоремы 2.1), что оператор $\Pi(w(t))$, порождаемый правой частью уравнения (3.17), переводит аналогичное $(2.22)$ множество из пространства $C([0, \bar{t}] ; E)$ в себя и является сжимающим (с константой сжатия порядка $\varepsilon$ ). А отсюда справедливость теоремы 3.1 вытекает уже очевидным образом.

Заканчивая обсуждение общих свойств краевой задачи (3.12), отметим еще одну характерную ее особенность. Непосредственная проверка с учетом равенств $(3.14),(3.15)$ показывает, что спектр оператора $L(\varepsilon)$ определяется из уравнений

$$
\lambda^{2}+\varepsilon\left(\frac{\nu \omega_{k}^{2}}{\sigma^{2}}-1-\frac{\nu}{\sigma^{2}}\right) \lambda+\omega_{k}^{2}=0, \quad k=1,2, \ldots,
$$

где, напомним, $\omega_{k}, k \geqslant 1,-$ собственные значения оператора $\mathscr{D}$ (см. (3.15)). Далее, нетрудно увидеть, что в случае $\nu>0$ корни уравнений (3.18) распадаются на две ветви - параболическую $\left\{\tilde{\lambda}_{k}(\varepsilon): \tilde{\lambda}_{k}(\varepsilon) / \omega_{k}^{2} \rightarrow-\varepsilon \nu / \sigma^{2}\right.$ при $\left.k \rightarrow \infty\right\}$ и гиперболическую $\left\{\tilde{\tilde{\lambda}}_{k}(\varepsilon): \tilde{\tilde{\lambda}}_{k}(\varepsilon) \rightarrow-\sigma^{2} /(\varepsilon \nu), k \rightarrow \infty\right\}$.

Из отмеченного характера поведения спектра и из формулы (3.16) следует, что при $\nu>0$ полугруппа $\exp (L(\varepsilon) t)$ является аналитической. А отсюда автоматически вытекает бесконечная дифференцируемость по $t$ при $t>0$ обобщенных решений, о которых говорится в теореме 3.1. Но в то же время из-за наличия гиперболической ветви в спектре оператора $L(\varepsilon)$ гладкость по пространственным переменным решений краевой задачи (3.12) при $t>0$ не увеличивается, оставаясь такой, как и при $t=0$. 
3.3. Основной результат. Для получения информации о существовании и устойчивости инвариантных торов краевой задачи (3.12) воспользуемся бесконечномерным аналогом известного асимптотического метода Крылова-Боголюбова-Митропольского [29]. В связи с этим сначала, привлекая разложение Фурье

$$
u=\sum_{n=1}^{\infty} u_{n}(t) e_{n}(x, y)
$$

перейдем от (3.12) к соответствующей счетной системе обыкновенных дифференциальных уравнений

$$
\ddot{u}_{n}+\varepsilon\left(\nu \lambda_{n}-1\right) \dot{u}_{n}+\omega_{n}^{2} u_{n}=-\varepsilon \dot{u}_{n} \sum_{k=1}^{\infty}\left(\dot{u}_{k}\right)^{2}, \quad n \geqslant 1,
$$

где, напомним, $\lambda_{n}$ и $\omega_{n}$ - собственные значения операторов $-\Delta$ и $\mathscr{D}$.

Суть упомянутого метода состоит в том, что возможные автоколебательные режимы системы (3.20) ищутся в виде

$$
u_{n}=u_{n, 0}(t, \tau)+\varepsilon u_{n, 1}(t, \tau)+\cdots, \quad \tau=\varepsilon t, \quad n \geqslant 1,
$$

где

$$
u_{n, 0}=z_{n}(\tau) \exp \left(i \omega_{n} t\right)+\bar{z}_{n}(\tau) \exp \left(-i \omega_{n} t\right)
$$

а подлежащие определению комплексные амплитуды $z_{n}(\tau), n \geqslant 1$, таковы, что сходится ряд с общим членом $\omega_{n}^{2}\left|z_{n}\right|^{2}$ (в этом случае после подстановки соотношений (3.22) вместо коэффициентов $u_{n}$ в ряд (3.19) получается функция класса $\stackrel{\circ}{W} \underset{2}{1}$ по переменным $x, y)$. Что же касается поправок порядка $\varepsilon$ из $(3.21)$, то фигурирующие в них функции $u_{n, 1}(t, \tau)$ будем искать в классе формальных тригонометрических рядов переменных $\omega_{k} t, k \geqslant 1$.

Итак, подставим в систему (3.20) разложения (3.21) и приравняем коэффициенты при $\varepsilon$. В результате приходим к счетной системе линейных неоднородных дифференциальных уравнений

$$
\ddot{u}_{n, 1}+\omega_{n}^{2} u_{n, 1}=f_{n}(t, \tau), \quad n \geqslant 1,
$$

где, как обычно, точкой обозначена производная по $t$, переменная $\tau$ считается параметром, а функции $f_{n}(t, \tau)$ имеют вид:

$$
\begin{gathered}
f_{n}=f_{n}^{0} \exp \left(i \omega_{n} t\right)+\overline{f_{n}^{0}} \exp \left(-i \omega_{n} t\right)-\dot{u}_{n, 0} \sum_{k=1}^{\infty}\left(\dot{u}_{k, 0}\right)^{2} \\
f_{n}^{0}=i \omega_{n}\left(1-\nu \lambda_{n}\right) z_{n}-2 i \omega_{n} \frac{d z_{n}}{d \tau}
\end{gathered}
$$

Необходимым и достаточным условием разрешимости уравнений (3.23) в интересующем нас классе функций является отсутствие в их правых частях (3.24) гармоник $\exp \left( \pm i \omega_{n} t\right)$. Добиться равенства нулю коэффициентов при указанных гармониках удается за счет имеющихся в запасе комплексных амплитуд $z_{n}$, для которых на этом пути получается некоторая счетная система обыкновенных дифференциальных уравнений. 
Наиболее просто упомянутая система для $z_{n}(\tau), n \geqslant 1$, записывается в случае, когда собственные значения $\lambda_{n}, n \geqslant 1$, оператора $-\Delta$ являются попарно различными, т. е.

$$
0<\lambda_{1}<\lambda_{2}<\cdots<\lambda_{n}<\lambda_{n+1}<\cdots .
$$

В самом деле, непосредственная проверка показывает, что при условиях (3.25), которые всюду ниже считаем выполненными, переменные $\xi_{n}=\omega_{n} z_{n}, n \geqslant 1$, удовлетворяют уравнениям

$$
2 \frac{d \xi_{n}}{d \tau}=\left[1-\nu \lambda_{n}-3\left|\xi_{n}\right|^{2}-2 \sum_{\substack{k=1 \\ k \neq n}}^{\infty}\left|\xi_{k}\right|^{2}\right] \xi_{n}, \quad n \geqslant 1 .
$$

Завершая описание алгоритмической части нашего метода, отметим, что система (3.26) обладает теми же общими свойствами, что и аналогичная ей система (1.12). А именно, она представляет собой абстрактную полулинейную параболическую систему в пространстве последовательностей $Z_{0}$ (см. п. 1.1), и, в частности, любое ее решение $\left\{\xi_{n}(\tau), n \geqslant 1\right\}$ с начальным условием

$$
\left(\xi_{1}(0), \bar{\xi}_{1}(0), \ldots, \xi_{n}(0), \bar{\xi}_{n}(0), \ldots\right)=\left(\xi_{1}^{0}, \overline{\xi_{1}^{0}}, \ldots, \xi_{n}^{0}, \overline{\xi_{n}^{0}}, \ldots\right) \in Z_{0}
$$

определено на полуоси $\tau \geqslant 0$. Поэтому если мы фиксируем какое-либо из таких решений и подставим соотношения $z_{n}(\tau)=\xi_{n}(\tau) / \omega_{n}, n \geqslant 1$, в (3.24), то все уравнения (3.23) станут разрешимыми в классе тригонометрических рядов, а их решения примут вид:

$$
\begin{aligned}
& u_{n, 1}=-\frac{i}{8 \omega_{n}^{2}}\left(\xi_{n}^{3} \exp \left(3 i \omega_{n} t\right)-\overline{\xi_{n}^{3}} \exp \left(-3 i \omega_{n} t\right)\right)+i \xi_{n} u_{n, 1}^{0}-i \bar{\xi}_{n} \overline{u_{n, 1}^{0}} \\
& u_{n, 1}^{0}=\sum_{\substack{k=1 \\
k \neq n}}^{\infty} \frac{1}{4 \omega_{k}}\left(\frac{\overline{\xi_{k}^{2}}}{\omega_{n}-\omega_{k}} \exp \left[i\left(\omega_{n}-2 \omega_{k}\right) t\right]-\frac{\xi_{k}^{2}}{\omega_{n}+\omega_{k}} \exp \left[i\left(\omega_{n}+2 \omega_{k}\right) t\right]\right) .
\end{aligned}
$$

Для придания изложенным формальным построениям необходимой строгости перейдем от (3.26) к аналогичной (1.15) системе

$$
\frac{d \rho_{n}}{d \tau}=\left[1-\nu \lambda_{n}-3 \rho_{n}-2 \sum_{\substack{k=1 \\ k \neq n}}^{\infty} \rho_{k}\right] \rho_{n}, \quad n \geqslant 1,
$$

где $\rho_{n}=\left|\xi_{n}\right|^{2}, n \geqslant 1$. Справедливо следующее утверждение.

ТЕОрема 3.2. Любому состоянию равновесия системы (3.28) с конечным числом ненулевых координат $\rho_{n_{j}}=\rho_{n_{j}}^{0}>0, j=1, \ldots, k$, экспоненииально устойчивому или дихотомичному, в исходной краевой задаче (3.12) при всех $\varepsilon \in\left(0, \varepsilon_{0}\right]$, где $\varepsilon_{0}>0$ достаточно мало, соответствует $k$-мерный инвариантный тор $T_{k}$ с теми же свойствами устойчивости. Указанный тор допускает представление вида

$$
\begin{gathered}
T_{k}: \quad u=\sum_{j=1}^{k}\left(\frac{2}{\omega_{n_{j}}} \sqrt{\rho_{n_{j}}^{0}} \cos \varphi_{j}+\varepsilon U_{j}\left(\varphi_{1}, \ldots, \varphi_{k}, \varepsilon\right)\right) e_{n_{j}}(x, y), \\
\dot{\varphi}_{j}=\omega_{n_{j}}+\varepsilon^{2} \Phi_{j}\left(\varphi_{1}, \ldots, \varphi_{k}, \varepsilon\right), \quad j=1, \ldots, k,
\end{gathered}
$$


где $U_{j}, \Phi_{j}$ - некоторые $2 \pi$-периодические по $\varphi_{1}, \ldots, \varphi_{k}$ функиии, имеющие при подходящем уменъшении $\varepsilon_{0}$ любую конечную гладкость по совокупности переменных $\left(\varphi_{1}, \ldots, \varphi_{k}, \varepsilon\right) \in[0,2 \pi] \times \cdots \times[0,2 \pi] \times\left[0, \varepsilon_{0}\right]$.

Справедливость сформулированной теоремы, как и аналогичных ей теорем 2.2, 2.3, вытекает из общих результатов монографии [24]. Впрочем, в данном случае в силу специфики нелинейности (3.6) проблема существования инвариантного тора (3.29) решается отдельно и сводится к конечномерной задаче. Действительно, нетрудно заметить, что система (3.20) имеет инвариантное подпространство $\left\{u_{n}=0, n \neq n_{j}, j=1, \ldots, k\right\}$, на котором она принимает вид

$$
\ddot{u}_{n_{j}}+\varepsilon\left(\nu \lambda_{n_{j}}-1\right) \dot{u}_{n_{j}}+\omega_{n_{j}}^{2} u_{n_{j}}=-\varepsilon \dot{u}_{n_{j}} \sum_{s=1}^{k}\left(\dot{u}_{n_{s}}\right)^{2}, \quad j=1, \ldots, k .
$$

Далее, применяя к (3.30) описанный выше асимптотический метод, приходим к аналогичной (3.28) амплитудной системе

$$
\frac{d \rho_{n_{j}}}{d \tau}=\left[1-\nu \lambda_{n_{j}}-3 \rho_{n_{j}}-2 \sum_{\substack{s=1 \\ s \neq j}}^{k} \rho_{n_{s}}\right] \rho_{n_{j}}, \quad j=1, \ldots, k,
$$

которая в силу условий теоремы допускает гиперболическое состояние равновесия $\left(\rho_{n_{1}}^{0}, \ldots, \rho_{n_{k}}^{0}\right)$. А отсюда и из известных результатов о бифуркации инвариантных торов в обыкновенных дифференциальных уравнениях (см., например, [29], [30]) заключаем, что интересующая нас система (3.30) имеет $k$-мерный инвариантный тор

$$
\begin{gathered}
\widetilde{T}_{k}: \quad u_{n_{j}}=\frac{2}{\omega_{n_{j}}} \sqrt{\rho_{n_{j}}^{0}} \cos \varphi_{j}+\varepsilon U_{j}\left(\varphi_{1}, \ldots, \varphi_{k}, \varepsilon\right), \\
\dot{\varphi}_{j}=\omega_{n_{j}}+\varepsilon^{2} \Phi_{j}\left(\varphi_{1}, \ldots, \varphi_{k}, \varepsilon\right), \quad j=1, \ldots, k,
\end{gathered}
$$

где функции $U_{j}, \Phi_{j}$ обладают всеми перечисленными в теореме свойствами. Остается добавить, что тору $\widetilde{T}_{k}$ в исходной краевой задаче (3.12) соответствует искомый тор (3.29).

Обратим внимание, что система (3.28) имеет по параметру $\nu$ ту же динамику, что и изученные ранее системы $(1.15),(2.69)$. В самом деле, как показывает непосредственная проверка, при выполнении неравенств (1.24), в которых критические значения $\bar{\nu}_{N}, \bar{\nu}_{N+1}$ теперь задаются формулами

$$
\bar{\nu}_{N}=\left[2\left(N \lambda_{N}-\sum_{k=1}^{N} \lambda_{k}\right)+\lambda_{N}\right]^{-1}, \quad N \geqslant 1,
$$

она допускает экспоненциально устойчивое состояние равновесия $O_{N}$ с координатами

$$
\begin{gathered}
\rho_{n}=\frac{1}{2 N+1}+\frac{2 \nu}{2 N+1} \sum_{k=1}^{N} \lambda_{k}-\nu \lambda_{n}, \quad n=1, \ldots, N ; \\
\rho_{n}=0 \quad \text { при } n \geqslant N+1 .
\end{gathered}
$$


Далее, при уменьшении параметра $\nu$ и при прохождении его через значение $\bar{\nu}_{N+1}$ состояние равновесия (3.31) теряет устойчивость и от него ответвляется очередное устойчивое состояние равновесия $O_{N+1}$. А это значит, что при $\nu \rightarrow 0$ наблюдается цепочка бифуркаций вида (2.72).

Из теоремы 3.2 и отмеченных динамических свойств системы (3.28) следует, что при $\nu \rightarrow 0$ в краевой задаче (3.12) происходит развитие турбулентности по Ландау. Однако, как и в случае возмущенного уравнения КдФ (см. (2.1)), мы можем доказать наличие хотя и любого наперед заданного, но конечного числа бифуркаций вида $T_{N} \rightarrow T_{N+1}$. Вопрос же о том, реализуется ли в рамках рассматриваемой модели при $\nu \rightarrow 0$ и при фиксированном $\varepsilon>0$ бесконечный каскад бифуркаций (1.62), остается открытым.

3.4. Существование турбулентного аттрактора. Одна из особенностей краевой задачи (3.12), выгодно отличающая ее от задачи (2.1), состоит в том, что здесь при $\nu=0, \varepsilon>0$ удается доказать наличие турбулентного аттрактора, свойства которого близки к описанным в п. 1.3. Причина, по которой это можно сделать, заключается в существовании при $\nu=0$ и при дополнительном ограничении

$$
\inf _{n, k \in \mathbb{N}, n \neq k}\left|\lambda_{n}-\lambda_{k}\right|=\delta_{0}>0
$$

на собственные значения оператора $-\Delta$ корректной замены переменных, приводящей исходную задачу (3.12) к системе вида

$$
\dot{\xi}=\Lambda_{0} \xi+\varepsilon \Lambda_{1}(\xi)+\varepsilon^{2} \Lambda_{*}(\xi, \varepsilon)
$$

в пространстве последовательностей $Z_{0}$. Здесь $\xi=\left(\xi_{1}, \bar{\xi}_{1}, \ldots, \xi_{n}, \bar{\xi}_{n}, \ldots\right) \in Z_{0}$,

$$
\begin{gathered}
\Lambda_{0}=\operatorname{diag}\left\{i \omega_{1},-i \omega_{1}, \ldots, i \omega_{n},-i \omega_{n}, \ldots\right\}, \\
\Lambda_{1}(\xi)=\left(\Lambda_{1,1}(\xi), \bar{\Lambda}_{1,1}(\xi), \ldots, \Lambda_{n, 1}(\xi), \bar{\Lambda}_{n, 1}(\xi), \ldots\right), \\
\Lambda_{n, 1}(\xi)=\frac{1}{2}\left[1-3\left|\xi_{n}\right|^{2}-2 \sum_{\substack{k=1 \\
k \neq n}}^{\infty}\left|\xi_{k}\right|^{2}\right] \xi_{n}, \quad n \geqslant 1,
\end{gathered}
$$

a оператор $\Lambda_{*}(\xi, \varepsilon)$ обладает следующим свойством: для любого натурального $m$ и произвольного ограниченного подмножества $B \subset Z_{0}$ найдется такое $\varepsilon_{0}=\varepsilon_{0}(m, B)>0$, что при каждом $\varepsilon \in\left[0, \varepsilon_{0}\right]$ он действует из $B$ в $Z_{0}$ и все его производные Фреше по $\xi$ до порядка $m$ включительно непрерывны и ограничены по совокупности переменных $(\xi, \varepsilon) \in B \times\left[0, \varepsilon_{0}\right]$.

Действительно, предположим, что нужная замена переменных уже построена и задача (3.12) преобразована к виду (3.33). Рассмотрим, далее, отвечающую (3.33) укороченную систему

$$
\dot{\xi}_{n}=i \omega_{n} \xi_{n}+\frac{\varepsilon}{2}\left[1-3\left|\xi_{n}\right|^{2}-2 \sum_{\substack{k=1 \\ k \neq n}}^{\infty}\left|\xi_{k}\right|^{2}\right] \xi_{n}, \quad n \geqslant 1,
$$

и выполним в ней последовательно замены $\xi_{n} \exp \left(-i \omega_{n} t\right) \rightarrow \xi_{n}, n \geqslant 1$, $\varepsilon t \rightarrow t$. В результате для $\rho_{n}=\left|\xi_{n}\right|^{2}, n \geqslant 1$, получим в точности систему (1.15) при 
$\nu=0$, имеющую турбулентный аттрактор $\widetilde{A}_{0}$. Тем самым, аналогичный аттрактор $A_{0}$ существует и у системы (3.35). А именно, любой точке $\rho_{0}=$ $\left(\rho_{1}^{0}, \ldots, \rho_{n}^{0}, \ldots\right) \in \widetilde{A}_{0}$ отвечает совокупность векторов $\xi \in A_{0}$ с компонентами $\xi_{n}=\sqrt{\rho_{n}^{0}} \exp \left(i \varphi_{n}\right), n \geqslant 1$, где вещественные параметры $\varphi_{n} \in[0,2 \pi]$ меняются независимо друг от друга.

Возвращаясь к полной системе (3.33) и учитывая описанные выше свойства оператора $\Lambda_{*}(\xi, \varepsilon)$, нетрудно проверить, что для ее траекторий оказывается инвариантным шар

$$
B=\left\{\xi \in Z_{0}: \sum_{n=1}^{\infty}\left|\xi_{n}\right|^{2} \leqslant 1\right\}
$$

Для этого достаточно взять функцию Ляпунова $V(\xi)=\sum_{n=1}^{\infty}\left|\xi_{n}\right|^{2}$ и убедиться, что

$$
\sum_{n=1}^{\infty} \frac{\partial V}{\partial \xi_{n}} \dot{\xi}_{n}+\frac{\partial V}{\partial \bar{\xi}_{n}} \dot{\bar{\xi}}_{n}=-\varepsilon-\varepsilon \sum_{n=1}^{\infty}\left|\xi_{n}\right|^{4}+O\left(\varepsilon^{2}\right) \text { равномерно по } \xi: \sum_{n=1}^{\infty}\left|\xi_{n}\right|^{2}=1 \text {. }
$$

А отсюда очевидным образом следует, что в множестве (3.36) система (3.33) имеет асимптотически близкий к $A_{0}$ аттрактор $A(\varepsilon)$, который и будем называть турбулентным.

Итак, проблема существования турбулентного аттрактора свелась к отысканию упомянутой выше замены переменных. При построении этой замены удобнее работать не с самой краевой задачей (3.12), а с эквивалентным ей уравнением (3.13), которое при $\nu=0$ приобретает вид

$$
\dot{w}=L_{0} w+\varepsilon L_{1} w+\varepsilon F(w),
$$

где $L_{0}=\mathscr{V}_{0} \mathscr{D}, L_{1}=\operatorname{diag}\{0,1\}$.

Покажем, что для уравнения (3.37) интересующая нас замена задается равенством

$$
w=\Theta_{0} \xi+\varepsilon \Theta_{1}(\xi), \quad \xi \in Z_{0},
$$

где линейный и нелинейный операторы $\Theta_{0}$ и $\Theta_{1}$ действуют из $Z_{0}$ в $E$ по правилам

$$
\begin{aligned}
\Theta_{0} \xi & =\sum_{n=1}^{\infty} \operatorname{colon}\left(\xi_{n}+\bar{\xi}_{n}, i \xi_{n}-i \bar{\xi}_{n}\right) e_{n}(x, y), \\
\Theta_{1}(\xi) & =\sum_{n=1}^{\infty} \operatorname{colon}\left(\theta_{n, 1}(\xi), \theta_{n, 2}(\xi)\right) e_{n}(x, y),
\end{aligned}
$$

а коэффициенты $\theta_{n, 1}, \theta_{n, 2}$ определяются следующим образом. Обратимся к равенствам (3.27) для $u_{n, 1}(t, \tau), n \geqslant 1$, и будем считать, что вместо $\xi_{n}(\tau), \xi_{k}(\tau)$, $k \neq n$, в них подставлены координаты $\xi_{n}, \xi_{k}, k \neq n$, произвольного не зависящего от $\tau$ вектора $\xi \in Z_{0}$. В результате получаются формальные тригонометрические ряды, которые обозначим через $u_{n, 1}(t, \xi), n \geqslant 1$, подчеркивая их зависимость от $\xi$. Используя эти ряды, положим

$$
\theta_{n, 1}(\xi)=\omega_{n} u_{n, 1}(0, \xi), \quad \theta_{n, 2}(\xi)=\frac{\partial u_{n, 1}}{\partial t}(0, \xi), \quad n \geqslant 1 .
$$


Корректность приведенной замены (3.38) устанавливается достаточно просто. Действительно, в силу (3.39) линейный оператор $\Theta_{0}$ является ограниченным вместе со своим обратным. Что же касается нелинейного слагаемого из (3.38), то, как нетрудно увидеть, равенства (3.40), (3.41) задают ограниченную кубическую форму, действующую из $Z_{0}$ в $E$.

Для проверки этого факта привлечем соотношения (3.27), (3.41) и воспользуемся свойствами (3.25), (3.32) собственных значений $\lambda_{n}, n \geqslant 1$. В результате приходим к оценкам

$$
\begin{aligned}
\left|\theta_{n, 1}(\xi)\right| & \leqslant \frac{1}{4 \omega_{n}}\left|\xi_{n}\right|^{3}+\frac{1}{2} \sum_{\substack{k=1 \\
k \neq n}}^{\infty}\left(\frac{2}{\omega_{k}}+\frac{1}{\left|\omega_{n}-\omega_{k}\right|}+\frac{1}{\omega_{n}+\omega_{k}}\right)\left|\xi_{k}\right|^{2}\left|\xi_{n}\right| \\
& \leqslant\left(\frac{5}{4 \omega_{1}}+\frac{1}{2 \delta_{0}}\right)\left|\xi_{n}\right| \sum_{k=1}^{\infty}\left|\xi_{k}\right|^{2}
\end{aligned}
$$

и к аналогичному неравенству

$$
\left|\theta_{n, 2}(\xi)\right| \leqslant\left(\frac{5}{4 \omega_{1}}+\frac{1}{2 \delta_{0}}\right)\left|\xi_{n}\right| \sum_{k=1}^{\infty}\left|\xi_{k}\right|^{2}
$$

А отсюда, в свою очередь, следует, что

$$
\left\|\Theta_{1}(\xi)\right\|_{E}=\left(\sum_{n=1}^{\infty}\left(\left|\theta_{n, 1}(\xi)\right|^{2}+\left|\theta_{n, 2}(\xi)\right|^{2}\right)\right)^{1 / 2} \leqslant\left(\frac{5}{4 \omega_{1}}+\frac{1}{2 \delta_{0}}\right)\left(\sum_{n=1}^{\infty}\left|\xi_{n}\right|^{2}\right)^{3 / 2} .
$$

Убедимся, наконец, что замена (3.38) действительно преобразует уравнение (3.37) к требуемому виду (3.33). С этой целью подставим в (3.37) соотношение (3.38), считая, что переменная $\xi$ дифференцируется по правилу (3.33). Приравняем затем в получившемся выражении коэффициенты при нулевой и первой степенях $\varepsilon$. В результате приходим к равенствам

$$
\Theta_{0} \Lambda_{0} \xi=L_{0} \Theta_{0} \xi, \quad \Theta_{1}^{\prime}(\xi) \Lambda_{0} \xi+\Theta_{0} \Lambda_{1}(\xi)=L_{0} \Theta_{1}(\xi)+L_{1} \Theta_{0} \xi+F\left(\Theta_{0} \xi\right)
$$

справедливость которых при любом $\xi$ непосредственно следует из способа определения операторов $\Theta_{j}, \Lambda_{j}, j=0,1$ (см. (3.34), (3.38)-(3.41)).

Тождества (3.42) гарантируют, что при выполнении замены (3.38) все "опасные" слагаемые, содержащие неограниченные операторы $\Lambda_{0}$ и $L_{0}$, сокращаются, а для оператора $\Lambda_{*}(\xi, \varepsilon)$ в итоге получается формула

$$
\begin{aligned}
\Lambda_{*}=\left(\Theta_{0}+\varepsilon \Theta_{1}^{\prime}(\xi)\right)^{-1}\left\{\varepsilon^{-1}\left[F\left(\Theta_{0} \xi+\varepsilon \Theta_{1}(\xi)\right)-F\left(\Theta_{0} \xi\right)\right]\right. \\
\left.+L_{1} \Theta_{1}(\xi)-\Theta_{1}^{\prime}(\xi) \Lambda_{1}(\xi)\right\} .
\end{aligned}
$$

Остается заметить, что из представления (3.43) нужные свойства ограниченности и гладкости этого оператора вытекают очевидным образом.

В заключение отметим, что на основании проделанного выше анализа модели (3.12) можно сделать следующие общие выводы. Уменьшение параметра $\nu$, отвечающего за социальный фактор в экономике и за ее государственное регулирование, приводит к развитию турбулентности. В реальности это означает, 
что экономические процессы становятся по существу непредсказуемыми. Если же наоборот, параметр $\nu$ излишне велик (жесткий контроль со стороны государства), то колебания отсутствуют, т.е. оказывается устойчивым нулевое состояние равновесия. И наконец, существует некий "средний" диапазон значений $\nu$, соответствующий нормальному экономическому функционированию, в котором краевая задача (3.12) имеет, например, устойчивый цикл или инвариантный тор невысокой размерности.

Добавим еще, что все установленные в данном параграфе результаты без каких-либо изменений переносятся, например, на краевую задачу

$$
u_{t t}-\varepsilon u_{t}-\varepsilon \nu \frac{\partial}{\partial t} \Delta u+u=\sigma^{2} \Delta u-\varepsilon u_{t} \cdot \iint_{\Omega}\left(u_{t}\right)^{2} d x d y,\left.\quad \frac{\partial u}{\partial \vec{n}}\right|_{\partial \Omega}=0,
$$

получающуюся из (3.8), (3.10) при ограничениях (3.11). Более того, несомненное преимущество нелинейности (3.6) перед ее стандартным аналогом (3.2) заключается в том, что конкретный вид граничных условий, при которых рассматривается уравнение (3.8), на самом деле несущественен. Важно лишь, чтобы собственные значения оператора $-\Delta$ при выбранных краевых условиях были попарно различными. Действительно, если это так, то система амплитудных уравнений, отвечающая за существование и устойчивость инвариантных торов, по-прежнему будет иметь вид (3.28).

\section{§ 4. Турбулентная буферность и ее математические модели}

4.1. Физическая постановка проблемы. Будем говорить, что в некоторой динамической системе реализуется турбулентная буферность, если при изменении управляющих параметров происходит неограниченный рост как количества ее сосуществующих устойчивых инвариантных торов, так и размерностей всех этих торов. Данный феномен очевидным образом связан со сценарием перехода к турбулентности по Ландау и наблюдается, например, когда при уменьшении вязкости или какого-либо другого параметра возникают все новые и новые цепочки бифуркаций вида (1.62).

Простейшей физической системой, в которой возможна турбулентная буферность, является двумерный прямоугольный массив идентичных осцилляторов, каждый из которых взаимодействует со своими соседями. Следуя работам [31], [32], предположим, что рассматриваемый массив имеет размер $N_{1} \times N_{2}$, где $N_{1}, N_{2}$ - пока произвольные натуральные числа, и состоит из одинаковых ячеек показанного на рис. 4.1 вида. Считаем, что центр $O$ каждой такой ячейки связан с землей посредством параллельно подключенных конденсатора $C_{0}$, индуктивности $L_{0}$ и туннельного диода с вольт-амперной характеристикой $i=f(u)$. Сами же ячейки взаимодействуют между собой через параллельно подсоединенные индуктивности $L$ и активные сопротивления $R$.

Для вывода математической модели описанной системы фиксируем ячейку с номерами $n, m$ и обозначим через $u_{n, m}(t)$ напряжение в ее узле $O$. Первый закон Кирхгофа для этого узла приводит к равенству

$$
i_{1}+i_{3}-i_{2}-i_{4}-i_{5}=0
$$




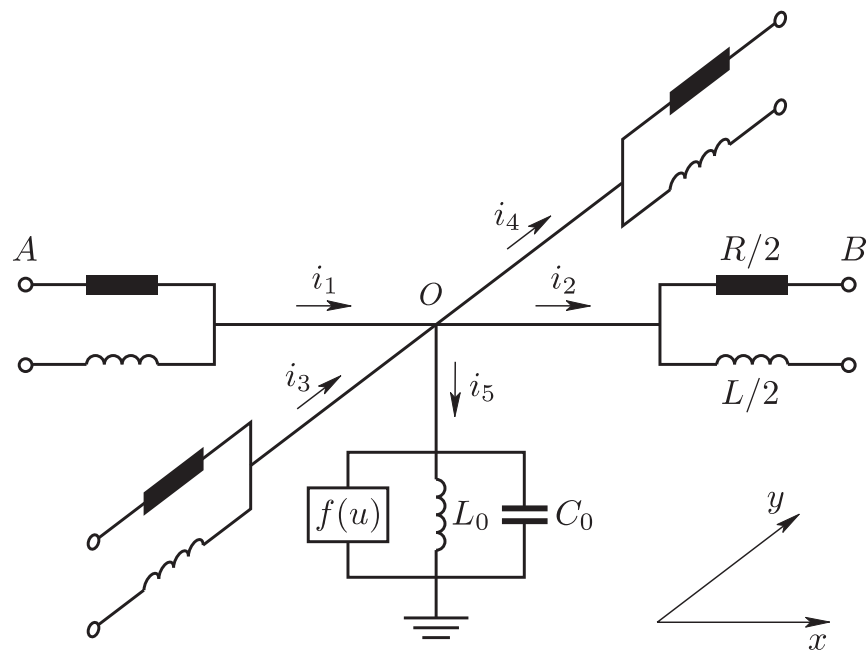

Рис. 4.1

где $i_{k}, k=1, \ldots, 5,-$ соответствующие токи (см. рис. 4.1). Далее, ток $i_{5}$ представляет собой сумму токов, текущих через конденсатор $C_{0}$, индуктивность $L_{0}$ и туннельный диод, и вследствие этого записывается в виде

$$
i_{5}=C_{0} \dot{u}_{n, m}(t)+f\left(u_{n, m}(t)\right)+\frac{1}{L_{0}} \int u_{n, m} d t .
$$

Что же касается остальных токов, то они в силу закона Ома для участков цепи задаются равенствами

$$
\begin{aligned}
& i_{1}=-\frac{1}{L} \int\left(u_{n, m}-u_{n-1, m}\right) d t-\frac{1}{R}\left(u_{n, m}-u_{n-1, m}\right), \\
& i_{2}=-\frac{1}{L} \int\left(u_{n+1, m}-u_{n, m}\right) d t-\frac{1}{R}\left(u_{n+1, m}-u_{n, m}\right), \\
& i_{3}=-\frac{1}{L} \int\left(u_{n, m}-u_{n, m-1}\right) d t-\frac{1}{R}\left(u_{n, m}-u_{n, m-1}\right), \\
& i_{4}=-\frac{1}{L} \int\left(u_{n, m+1}-u_{n, m}\right) d t-\frac{1}{R}\left(u_{n, m+1}-u_{n, m}\right) .
\end{aligned}
$$

Приведенные соотношения (4.1)-(4.3) позволяют выписать систему, связывающую напряжения $u_{n, m}(t), n=1, \ldots, N_{1}, m=1, \ldots, N_{2}$, в узлах массива. Действительно, подставляя формулы (4.2), (4.3) в (4.1) и дифференцируя результат по $t$, приходим к уравнениям

$$
\begin{aligned}
& \frac{1}{L}\left(u_{n+1, m}-2 u_{n, m}+u_{n-1, m}\right)+\frac{1}{L}\left(u_{n, m+1}-2 u_{n, m}+u_{n, m-1}\right) \\
&+\frac{1}{R}\left(\dot{u}_{n+1, m}-2 \dot{u}_{n, m}+\dot{u}_{n-1, m}\right)+\frac{1}{R}\left(\dot{u}_{n, m+1}-2 \dot{u}_{n, m}+\dot{u}_{n, m-1}\right) \\
&=C_{0} \ddot{u}_{n, m}+\frac{d}{d t} f\left(u_{n, m}\right)+\frac{1}{L_{0}} u_{n, m}, \quad n=1, \ldots, N_{1}, \quad m=1, \ldots, N_{2} .
\end{aligned}
$$


Получившуюся систему (4.4) следует дополнить какими-либо граничными условиями. В работе [31] рассматривалась ситуация, когда две смежные стороны граничного прямоугольника заземлены, а две другие - свободны. Далее, в [32] изучался случай трех заземленных сторон и одной свободной. Мы же, следуя [33], остановимся на простейшем варианте

$$
\begin{aligned}
u_{N_{1}+1, m} & =u_{0, m}=0, \quad m=1, \ldots, N_{2} ; \\
u_{n, N_{2}+1} & =u_{n, 0}=0, \quad n=1, \ldots, N_{1},
\end{aligned}
$$

означающем, что заземлены все граничные ячейки.

Всюду ниже предполагается, что каждая отдельно взятая ячейка массива имеет одинаковые линейные размеры по осям $x, y$ и, в частности, расстояние между показанными на рис. 4.1 точками $A$ и $B$ равно $h$. Далее, как и в статьях [31], [34], будем считать, что

$$
C_{0}=C_{0, *} h^{2}, \quad L_{0}=\frac{L_{0, *}}{h^{2}}, \quad R=R_{*}, \quad L=L_{*}, \quad f(u)=-h^{2} G_{*}\left(u-\frac{u^{3}}{3}\right),
$$

где электрические параметры $C_{0, *}, L_{0, *}, R_{*}, L_{*}, G_{*}$ уже не зависят от $h$. И наконец, уточним правило выбора натуральных чисел $N_{1}, N_{2}$, а также способ введения координат $x, y$ на плоскости, проходящей через центры всех ячеек. $\mathrm{C}$ этой целью фиксируем произвольно постоянные $a_{1}, a_{2}>0$, затем положим

$$
N_{1}=\left[\frac{a_{1}}{h}\right], \quad N_{2}=\left[\frac{a_{2}}{h}\right] \quad([\cdot]-\text { целая часть })
$$

и будем считать, что узлу $O$ ячейки с номерами $n, m$ соответствует точка $(x, y)=(n h, m h)$.

Соотношения (4.6), (4.7) и указанный способ введения $x, y$ позволяют перейти от (4.4), (4.5) к соответствующей распределенной модели. Для того чтобы сделать это, поделим каждое уравнение системы (4.4) на $h^{2}$ и устремим параметр $h$ к нулю. В результате для распределенного напряжения $u=u(t, x, y)$ получаем краевую задачу

$$
C_{0, *} u_{t t}-G_{*}\left(1-u^{2}\right) u_{t}+\frac{1}{L_{0, *}} u=\frac{1}{L_{*}} \Delta u+\frac{1}{R_{*}} \frac{\partial}{\partial t} \Delta u,\left.\quad u\right|_{\partial \Omega_{0}}=0
$$

в области $\Omega_{0}=\left\{(x, y): 0 \leqslant x \leqslant a_{1}, 0 \leqslant y \leqslant a_{2}\right\}$. В дальнейшем, однако, по чисто техническим причинам удобнее работать с задачей

$$
u_{t t}-\varepsilon u_{t}-\varepsilon \nu \frac{\partial}{\partial t} \mathscr{L} u+u=\mathscr{L} u-\varepsilon u^{2} u_{t},\left.\quad u\right|_{\partial \Omega}=0
$$

где $\mathscr{L}=\sigma_{1}^{2} \partial^{2} / \partial x^{2}+\sigma_{2}^{2} \partial^{2} / \partial y^{2}, \varepsilon=G_{*} \sqrt{L_{0, *} / C_{0, *}}, \nu=\sqrt{L_{*} / L_{0, *}} /\left(R_{*} G_{*}\right)$,

$$
\sigma_{j}=\frac{1}{a_{j}} \sqrt{L_{0, *} / L_{*}}, \quad j=1,2 ; \quad \Omega=\{(x, y): 0 \leqslant x \leqslant 1,0 \leqslant y \leqslant 1\},
$$

получающейся из (4.8) в результате нормировок $t / \sqrt{L_{0, *} C_{0, *}} \rightarrow t, x / a_{1} \rightarrow x$, $y / a_{2} \rightarrow y$. 
Следует отметить, что хотя краевая задача (4.9) выводится из простых физических соображений, но на самом деле она служит феноменологической моделью нелинейных процессов в системах из различных областей естествознания. K таковым согласно [33] относятся всевозможные триггерные системы, используемые в компьютерах в качестве запоминающего устройства, многомодовые лазеры, в которых несколько оптических мод конкурируют между собой с целью получения энергии от активной среды, взаимодействующие биологические виды с общим источником пищи, неокортекс человеческого мозга, в котором множество концепций соревнуются друг с другом в целях доминирования, и т. д. Для нас же эта задача представляет интерес в первую очередь в связи с тем, что при некоторых условиях на параметры $\sigma_{1}, \sigma_{2}$ из (4.10) и при согласованном стремлении к нулю $\varepsilon$ и $\nu$ в ней реализуется турбулентная буферность.

4.2. Существование и устойчивость инвариантных торов. Нетрудно увидеть, что краевая задача (4.9), рассматриваемая как динамическая система в фазовом пространстве $\left(u, u_{t}\right) \in \stackrel{\circ}{W} \underset{2}{2}(\Omega) \times \stackrel{\circ}{W} \underset{2}{2}(\Omega)$, обладает теми же общими свойствами, что и изученная выше задача (3.12). В частности, при $\varepsilon=0$ она допускает тригонометрические решения

$$
\begin{gathered}
u=\exp \left( \pm i \omega_{n, k} t\right) e_{n, k}(x, y), \quad e_{n, k}(x, y)=2 \sin n \pi x \sin k \pi y, \\
\omega_{n, k}=\sqrt{1+\pi^{2}\left(\sigma_{1}^{2} n^{2}+\sigma_{2}^{2} k^{2}\right)}, \quad n, k \in \mathbb{N} .
\end{gathered}
$$

Для получения как можно более полного представления о динамике краевой задачи (4.9) по параметру $\nu$ воспользуемся тем же методом, что и в п. 3.3. В данном случае, обыгрывая существование гармонических решений (4.11), при условиях $0<\varepsilon \ll 1, \nu=$ const $>0$ ее автоколебания будем искать в виде

$$
u=u_{0}(t, \tau, x, y)+\varepsilon u_{1}(t, \tau, x, y)+\cdots, \quad \tau=\varepsilon t,
$$

где

$$
u_{0}=\sum_{n, k=1}^{\infty}\left[z_{n, k}(\tau) \exp \left(i \omega_{n, k} t\right)+\bar{z}_{n, k}(\tau) \exp \left(-i \omega_{n, k} t\right)\right] e_{n, k}(x, y),
$$

а пока неизвестные комплексные амплитуды $z_{n, k}$ таковы, что сходится ряд с общим членом $\omega_{n, k}^{4}\left|z_{n, k}\right|^{2}$ (в этом случае $\left.u_{0} \in \stackrel{\circ}{W_{2}^{2}}(\Omega)\right)$.

Подставляя (4.12), (4.13) в (4.9) и приравнивая коэффициенты при $\varepsilon$, для $u_{1}$ получаем краевую задачу

$$
\frac{\partial^{2} u_{1}}{\partial t^{2}}+u_{1}-\mathscr{L} u_{1}=\frac{\partial}{\partial t}\left[u_{0}+\nu \mathscr{L} u_{0}-\frac{u_{0}^{3}}{3}-2 \frac{\partial u_{0}}{\partial \tau}\right],\left.\quad u_{1}\right|_{\partial \Omega}=0 .
$$

Распорядимся, далее, выбором параметров $\sigma_{1}$ и $\sigma_{2}$ таким образом, чтобы между собственными частотами $\omega_{n, k}$ из (4.11) отсутствовали какие бы то ни было резонансные соотношения третьего порядка, т. е. чтобы было исключено одновременное выполнение равенств вида

$$
\begin{gathered}
\omega_{n_{0}, k_{0}}=m_{1} \omega_{n_{1}, k_{1}}+m_{2} \omega_{n_{2}, k_{2}}+m_{3} \omega_{n_{3}, k_{3}}, \\
n_{0}= \pm n_{1} \pm n_{2} \pm n_{3}, \quad k_{0}= \pm k_{1} \pm k_{2} \pm k_{3},
\end{gathered}
$$


для любого набора индексов $\left(n_{j}, k_{j}\right), j=0,1,2,3$, для любого целочисленного вектора $\left(m_{1}, m_{2}, m_{3}\right)$ такого, что $\left|m_{1}\right|+\left|m_{2}\right|+\left|m_{3}\right|=3$, и при любой расстановке знаков во втором и третьем из этих соотношений (тождественные резонансы $\omega_{n, k}=\omega_{n, k}+\omega_{m, s}-\omega_{m, s}$ из рассмотрения, естественно, исключаются). Тогда функция $u_{1}$ может быть найдена в виде формального тригонометрического ряда в том и только том случае, если в неоднородности из (4.14) отсутствуют гармоники (4.11). Поэтому приравняем коэффициенты при этих гармониках к нулю. В итоге для амплитуд $z_{n, k}$ приходим к системе

$$
\begin{gathered}
2 \frac{d z_{n, k}}{d \tau}=\left(1-\nu\left(\omega_{n, k}^{2}-1\right)\right) z_{n, k}-\frac{9}{4} z_{n, k}\left|z_{n, k}\right|^{2}-3 z_{n, k} \sum_{\substack{m=1 \\
m \neq n}}^{\infty}\left|z_{m, k}\right|^{2} \\
-3 z_{n, k} \sum_{\substack{m=1 \\
m \neq k}}^{\infty}\left|z_{n, m}\right|^{2}-2 z_{n, k} \sum_{\substack{m, r=1 \\
m \neq n, r \neq k}}^{\infty}\left|z_{m, r}\right|^{2}, \quad n, k \in \mathbb{N} .
\end{gathered}
$$

Однако, как и в п. 3.3, в дальнейшем будем иметь дело с системой для $\rho_{n, k}=$ $\left|z_{n, k}\right|^{2}$, записывающейся в виде

$$
\begin{aligned}
\frac{d \rho_{n, k}}{d \tau}=( & 1-\nu\left(\omega_{n, k}^{2}-1\right)-\frac{9}{4} \rho_{n, k}-3 \sum_{m \neq k} \rho_{n, m} \\
& \left.-3 \sum_{m \neq n} \rho_{m, k}-2 \sum_{m \neq n, r \neq k} \rho_{m, r}\right) \rho_{n, k}, \quad n, k \in \mathbb{N} .
\end{aligned}
$$

Предположим, что система (4.15), рассматриваемая в пространстве последовательностей $\rho=\left\{\rho_{n, k}, n, k \in \mathbb{N}\right\}$ с нормой

$$
\|\rho\|=\sum_{n, k=1}^{\infty} \omega_{n, k}^{4}\left|\rho_{n, k}\right|<\infty,
$$

имеет состояние равновесия с конечным числом ненулевых положительных координат

$$
\rho_{n_{1}, k_{1}}, \ldots, \rho_{n_{p}, k_{p}}, \quad p \geqslant 1 .
$$

Тогда оказывается справедливым следующее утверждение [24], [25].

ТЕОрема 4.1. Любому состоянию равновесия вида (4.16) системы (4.15), экспоненииально устойчивому или дихотомичному, в исходной задаче (4.9) при всех достаточно малых $\varepsilon>0$ соответствует $p$-мерный инвариантный тор той же устойчивости, задающийся равенствами:

$$
\begin{gathered}
u=\sum_{j=1}^{p} 2 \sqrt{\rho_{n_{j}, k_{j}}} \cos \varphi_{j} e_{n_{j}, k_{j}}(x, y)+\varepsilon U_{*}(\varphi, x, y, \varepsilon), \\
\frac{d \varphi}{d t}=\omega+\varepsilon^{2} \Phi_{*}(\varphi, \varepsilon) .
\end{gathered}
$$

Здесъ $\varphi=\operatorname{colon}\left(\varphi_{1}, \ldots, \varphi_{p}\right), \omega=\operatorname{colon}\left(\omega_{n_{1}, k_{1}}, \ldots, \omega_{n_{p}, k_{p}}\right)$, a $2 \pi$-nериодические по $\varphi$ функции $U_{*}, \Psi_{*}$ и любое фиксированное количество их производных по $\varphi$ ограничены равномерно по $\varphi, \varepsilon$ в метрике $\stackrel{\circ}{W} \underset{2}{2}(\Omega)$ u $\mathbb{R}^{p}$ соответственно. 
Итак, вопрос о динамике краевой задачи (4.9) по параметру $\nu$ сводится к аналогичному вопросу для соответствующей амплитудной системы (4.15). В связи с этим обратим внимание на две характерные особенности последней. Отметим, во-первых, что при любом фиксированном $\nu>0$ за ее аттракторы в инвариантном конусе $K=\left\{\rho_{n, k}: \rho_{n, k} \geqslant 0\right\}$ отвечает конечномерная система, получающаяся из (4.15), если положить равными нулю компоненты $\rho_{n, k}$, для которых $1-\nu\left(\omega_{n, k}^{2}-1\right)<0$. Действительно, из структуры входящих в $(4.15)$ уравнений следует, что упомянутые компоненты заведомо стремятся к нулю при $\tau \rightarrow \infty$. Очевидно также, что при $\nu \rightarrow 0$ размерность соответствующей конечномерной системы неограниченно растет.

Во-вторых, при $\nu=0$ любое состояние равновесия системы (4.15) с конечным числом ненулевых положительных координат (4.16) экспоненциально неустойчиво, причем бесконечна размерность его неустойчивого многообразия (напомним, что такая же ситуация наблюдается при $\nu=0$ и в системе (1.15)). Для того чтобы убедиться в этом, положим $\eta_{j}=\rho_{n_{j}, k_{j}}, j=1, \ldots, p$, и рассмотрим систему для нахождения $\eta_{j}$, имеющую вид

$$
\alpha_{j, 1} \eta_{1}+\alpha_{j, 2} \eta_{2}+\cdots+\alpha_{j, p} \eta_{p}=1, \quad j=1, \ldots, p,
$$

где $\alpha_{j, j}=9 / 4, j=1, \ldots, p$, а все остальные $\alpha_{j, k}, j \neq k$, равны 2 или 3. Тем самым, заменяя в (4.18) все $\alpha_{j, k}$ при $j \neq k$ на 2 , приходим к серии оценок

$$
2 \eta_{1}+\cdots+2 \eta_{j-1}+9 \frac{\eta_{j}}{4}+2 \eta_{j+1}+\cdots+2 \eta_{p} \leqslant 1, \quad j=1, \ldots, p .
$$

Складывая, далее, все неравенства (4.19), убеждаемся, что

$$
S \equiv \sum_{j=1}^{p} \eta_{j} \leqslant \frac{4 p}{8 p+1} .
$$

И наконец, рассмотрим систему в вариациях на интересующем нас состоянии равновесия. Нетрудно заметить, что любое ее уравнение с индексами $(m, r)$, для которых $m \neq n_{j}$ и $r \neq k_{j}, j=1, \ldots, p$, имеет вид

$$
\frac{d h_{m, r}}{d \tau}=(1-2 S) h_{m, r}
$$

А отсюда и из оценки

$$
1-2 S \geqslant \frac{1}{8 p+1}
$$

(см. (4.20)) требуемый факт вытекает очевидным образом.

Введем в рассмотрение отвечающую (4.15) укороченную систему

$$
\begin{aligned}
\frac{d \rho_{n, k}}{d \tau}=\left(1-\nu\left(\omega_{n, k}^{2}-1\right)-\frac{9}{4} \rho_{n, k}-3 \sum_{\substack{m=1 \\
m \neq k}}^{N} \rho_{n, m}\right. \\
\left.\quad-3 \sum_{\substack{m=1 \\
m \neq n}}^{N} \rho_{m, k}-2 \sum_{\substack{m, r=1 \\
m \neq n, r \neq k}}^{N} \rho_{m, r}\right) \rho_{n, k}, \quad n, k=1, \ldots, N,
\end{aligned}
$$


предполагая номер $N=N(\nu)$ настолько большим, что $1-\nu\left(\omega_{n, k}^{2}-1\right)<0$ при всех $(n, k)$, для которых $n \geqslant N+1$ или $k \geqslant N+1$. Рассмотрим, далее, все экспоненциально устойчивые состояния равновесия (если они существуют) системы (4.22), лежащие в конусе $K$, и обозначим их количество через $N_{*}=N_{*}(\nu)$. Кроме этого, каждому такому состоянию равновесия поставим в соответствие число $p \geqslant 0$, равное количеству его ненулевых координат, и положим $N_{* *}(\nu)=\min p$ (минимум берется по всем устойчивым состояниям равновесия из $K)$. Указанные выше характерные особенности системы (4.15) наводят на мысль о справедливости следующего утверждения.

ТеОрема 4.2. Имеют место предельные равенства

$$
\lim _{\nu \rightarrow 0} N_{*}(\nu)=\lim _{\nu \rightarrow 0} N_{* *}(\nu)=\infty .
$$

ДокАЗАТЕЛЬство. Обоснование первого из предельных равенств (4.23) носит конструктивный характер. А именно, при каждом достаточно малом $\nu>0$ будет предъявлен конкретный набор устойчивых состояний равновесия системы (4.15), количество которых неограниченно возрастает при $\nu \rightarrow 0$.

Рассмотрим числовую последовательность

$$
\bar{\nu}_{N}=\frac{3}{\pi^{2}\left(\sigma_{1}^{2}+\sigma_{2}^{2}\right) N\left(16 N^{2}-9 N-4\right)}, \quad N \geqslant 1,
$$

и будем считать, что параметр $\nu$ удовлетворяет неравенствам вида (1.24). Несложная проверка показывает, что в этом случае система (4.15) имеет группу состояний равновесия, у которых ненулевыми являются только координаты

$$
\rho_{j, k_{j}}=\eta_{j}, \quad \eta_{j}=4\left[1-2 S-\nu \pi^{2}\left(\sigma_{1}^{2} j^{2}+\sigma_{2}^{2} k_{j}^{2}\right)\right]>0, \quad j=1, \ldots, N,
$$

где

$$
S=\frac{4 N}{8 N+1}\left[1-\frac{\nu \pi^{2}}{6}\left(\sigma_{1}^{2}+\sigma_{2}^{2}\right)(N+1)(2 N+1)\right],
$$

a $\left(k_{1}, \ldots, k_{N}\right)$ - всевозможные перестановки, получающиеся из целочисленного вектора $(1, \ldots, N)$. Количество же таких состояний равновесия равно, очевидно, $N$ ! .

Анализ свойств устойчивости состояний равновесия (4.24), (4.25) подробно изложен в монографиях [24], [25] (см. также статьи [35], [36]). Поэтому здесь соответствующие выкладки опустим. Отметим только, что каждое из них устойчиво при $\nu \in\left(\bar{\nu}_{N+1}, \bar{\nu}_{N}\right)$. Далее, при $\nu=\bar{\nu}_{N}$ обращаются в нуль координаты $\rho_{N, k_{N}}$ у тех состояний равновесия $(4.24),(4.25)$, для которых $k_{N}=N$ (количество таких состояний равновесия равно $(N-1) !)$. Что же касается оставшихся $N !-(N-1)$ ! положений равновесия семейства $(4.24),(4.25)$, то они сохраняют свою устойчивость и при $\nu=\bar{\nu}_{N}$. А отсюда очевидным образом вытекает требуемое предельное равенство $\lim _{\nu \rightarrow 0} N_{*}(\nu)=\infty$.

Для обоснования второго предельного равенства (4.23) достаточно показать, что для любого натурального $p$ найдется такое $\nu_{0}=\nu_{0}(p)>0$, что при всех $0<$ $\nu \leqslant \nu_{0}$ каждое состояние равновесия системы (4.15) с количеством ненулевых положительных координат, равным $p$, экспоненциально неустойчиво. 
В предположении противного существуют такое натуральное $p_{0}$ и такая последовательность $\nu_{m}>0, \nu_{m} \rightarrow 0$ при $m \rightarrow \infty$, что при $\nu=\nu_{m}$ система (4.15) имеет в конусе $K$ хотя бы одно устойчивое положение равновесия с конечным числом ненулевых координат вида

$$
\rho_{n_{1}, k_{1}}, \rho_{n_{2}, k_{2}}, \ldots, \rho_{n_{p_{0}}, k_{p_{0}}} \cdot
$$

Заметим, что, переходя, если это необходимо, от $\nu_{m}$ к соответствующей подпоследовательности, всегда можно добиться выполнения для номеров $n_{j}=$ $n_{j}\left(\nu_{m}\right), k_{j}=k_{j}\left(\nu_{m}\right), j=1, \ldots, p_{0}$, предельных равенств

$$
\lim _{m \rightarrow \infty} n_{j}=n_{j}^{0}, \quad \lim _{m \rightarrow \infty} k_{j}=k_{j}^{0}, \quad j=1, \ldots, p_{0},
$$

где $n_{j}^{0}, k_{j}^{0}$ могут быть как конечными натуральными числами, так и равняться бесконечности.

Положим $N_{0}=\max \left\{n_{j}^{0}, k_{j}^{0}\right\}$, где максимум берется по конечным предельным значениям из (4.27) (если таковых нет, то полагаем $N_{0}=0$ ). Линеаризуем затем систему (4.15) при $\nu=\nu_{m}$ на состоянии равновесия (4.26) и выпишем уравнение получившейся системы в вариациях с номерами $\left(N_{0}+1, N_{0}+1\right)$. Из способа выбора $N_{0}$ следует, что при всех достаточно больших $m$ это уравнение принимает вид

$$
\frac{d h_{N_{0}+1, N_{0}+1}}{d \tau}=\left[1-2 S-\left(N_{0}+1\right)^{2}\left(\sigma_{1}^{2}+\sigma_{2}^{2}\right) \nu_{m} \pi^{2}\right] h_{N_{0}+1, N_{0}+1},
$$

где, как и выше, $S=\sum_{j=1}^{p_{0}} \rho_{n_{j}, k_{j}}$, причем сохраняется (вместе с доказательством) аналогичная (4.21) оценка

$$
1-2 S \geqslant \frac{1}{8 p_{0}+1}
$$

Остается заметить, что в силу (4.29) для коэффициента уравнения (4.28) при всех достаточно больших $m$ справедливо неравенство

$$
1-2 S-\left(N_{0}+1\right)^{2}\left(\sigma_{1}^{2}+\sigma_{2}^{2}\right) \nu_{m} \pi^{2}>0
$$

противоречащее предполагаемой устойчивости состояний равновесия (4.26). Теорема 4.2 полностью доказана.

Из теорем 4.1, 4.2 следует, что при некоторой общности положения, связанной с выбором параметров $\sigma_{1}, \sigma_{2}$, и при $\nu \rightarrow 0, \varepsilon \rightarrow 0$ в краевой задаче $(4.9)$ реализуется интересующий нас феномен турбулентной буферности. Действительно, в этом случае неограниченно возрастает количество ее устойчивых инвариантных торов вида (4.17), причем одновременно неограниченно увеличиваются и размерности всех этих торов.

Подводя итог, отметим, что в отличие от краевой задачи (3.12) здесь при $\nu=0, \varepsilon>0$ не удается доказать существование турбулентного аттрактора. Причиной этого являются так называемые приближенные резонансы, от которых нельзя избавиться за счет шевеления параметров $\sigma_{1}, \sigma_{2}$ и которые, в свою 
очередь, приводят к эффекту малых знаменателей. Для примера обратим внимание, что в знаменателе коэффициента функции $u_{1}(t, \tau, x, y)$ при гармонике $\exp \left(i \omega_{m, s} t\right) e_{m-2 n, s+2 k}(x, y)$ фигурирует выражение

$$
\frac{\sigma_{1}^{2} m n-\sigma_{2}^{2} s k-\sigma_{1}^{2} n^{2}-\sigma_{2}^{2} k^{2}}{\omega_{m, s}},
$$

стремящееся к 0 при фиксированных $n, k$ и при $m, s \rightarrow \infty, s / m \rightarrow \sigma_{1}^{2} n /\left(\sigma_{2}^{2} k\right)$. А это значит, что в данном случае формула $u=u_{0}+\varepsilon u_{1}$ уже не порождает корректной замены переменных, действующей из $\stackrel{\circ}{W} \underset{2}{2}(\Omega) \times \stackrel{\circ}{W} \underset{2}{1}(\Omega)$ в подходящее пространство последовательностей.

4.3. О других моделях турбулентной буферности. Весьма интересно, что краевая задача (4.9) допускает экономическую интерпретацию. Действительно, нетрудно увидеть, что она получается из экономической модели (3.5) в случае нелинейности (3.2), прямоугольной области $\Omega$, граничных условий (3.9) и прежних предположений (3.11) относительно параметров $a, b$, $d_{1}, d_{2}$.

Из общих соображений ясно, что обнаруженный в краевой задаче (4.9) феномен турбулентной буферности достаточно универсален и присущ не только волновым уравнениям. Для того чтобы убедиться в этом, покажем, что при определенных условиях он наблюдается, например, в уравнении Гинзбурга-Ландау (1.65), которое само является фундаментальным во многих вопросах математической физики (см. обзор [37], где содержится достаточно подробная библиография по этой тематике).

Рассмотрим краевую задачу, получающуюся из уравнения (1.65) в прямоугольнике $\left\{(x, y): 0 \leqslant x \leqslant a_{1}, 0 \leqslant y \leqslant a_{2}\right\}$ с нулевыми граничными условиями Дирихле, и будем считать параметры $c_{j}, j=1,2,3$, в ней таковыми, что

$c_{1}=\nu, \quad \nu=$ const $>0 ; \quad c_{2}=-\frac{1}{\varepsilon}, \quad 0<\varepsilon \ll 1 ; \quad c_{3}=c_{0}, \quad c_{0}=$ const $\in \mathbb{R}$.

В результате после нормировок $t / \varepsilon \rightarrow t, x / a_{1} \rightarrow x, y / a_{2} \rightarrow y$ получаем краевую задачу в единичном квадрате $\Omega$, имеющую вид:

$$
w_{t}=(\varepsilon \nu-i) \mathscr{L} w+\varepsilon\left[w-\left(1+i c_{0}\right)|w|^{2} w\right],\left.\quad w\right|_{\partial \Omega}=0,
$$

где оператор $\mathscr{L}$ тот же самый, что и в $(4.9)$, но теперь $\sigma_{j}=1 / a_{j}, j=1,2$.

Поставим вопрос о динамике краевой задачи (4.30) по параметру $\nu$, считая, что $(\operatorname{Re} w, \operatorname{Im} w) \in \stackrel{\circ}{W} \underset{2}{2}(\Omega) \times \stackrel{\circ}{W} \underset{2}{2}(\Omega)$. В связи с этим обратим внимание, что при $\varepsilon=0$ она допускает волновые решения

$$
w=\exp \left(i \omega_{n, k} t\right) e_{n, k}(x, y), \quad \omega_{n, k}=\pi^{2}\left(\sigma_{1}^{2} n^{2}+\sigma_{2}^{2} k^{2}\right), \quad n, k \geqslant 1,
$$

где функции $e_{n, k}(x, y)$ те же, что и в (4.11). Тем самым, к ней можно применить изложенную в п. 4.2 методику исследования автоколебаний.

Действительно, наличие волновых решений (4.31) позволяет искать возможные автоколебательные режимы краевой задачи (4.30) в виде формального ряда

$$
w=w_{0}(t, \tau, x, y)+\varepsilon w_{1}(t, \tau, x, y)+\cdots, \quad \tau=\varepsilon t,
$$


где

$$
w_{0}=\sum_{n, k=1}^{\infty} z_{n, k}(\tau) \exp \left(i \omega_{n, k} t\right) e_{n, k}(x, y),
$$

а подлежащие определению комплексные амплитуды $z_{n, k}$ удовлетворяют требованию $\sum_{n, k} \omega_{n, k}^{2}\left|z_{n, k}\right|^{2}<\infty$, обеспечивающему принадлежность функции (4.33) пространству $\stackrel{\circ}{W} 2(\Omega)$.

После подстановки соотношений (4.32), (4.33) в (4.30) и последующего приравнивания коэффициентов при $\varepsilon$ для функции $w_{1}$ получаем линейную неоднородную краевую задачу

$$
\frac{\partial w_{1}}{\partial t}+i \mathscr{L} w_{1}=g(t, \tau, x, y),\left.\quad w_{1}\right|_{\partial \Omega}=0,
$$

в которой $g=-\partial w_{0} / \partial \tau+\nu \mathscr{L} w_{0}+w_{0}-\left(1+i c_{0}\right)\left|w_{0}\right|^{2} w_{0}$, а переменная $\tau$ рассматривается как параметр.

Дальнейший анализ проведем в предположении об иррациональности числа $\sigma_{1}^{2} / \sigma_{2}^{2}$. В этом случае невозможно одновременное выполнение равенств вида

$$
\omega_{n, k}=\omega_{n_{1}, k_{1}}+\omega_{n_{2}, k_{2}}-\omega_{n_{3}, k_{3}}, \quad n= \pm n_{1} \pm n_{2} \pm n_{3}, \quad k= \pm k_{1} \pm k_{2} \pm k_{3}
$$

при произвольном выборе комбинаций знаков "+" и “-” во втором и третьем из них (как и в п. 4.2, тождественные резонансы, когда $(n, k)=\left(n_{1}, k_{1}\right)$, $\left(n_{2}, k_{2}\right)=\left(n_{3}, k_{3}\right)$, здесь не рассматриваются). А отсюда, как нетрудно увидеть, следует, что краевая задача (4.34) будет разрешимой в классе формальных тригонометрических рядов в том и только том случае, когда в неоднородности $g$ отсутствуют гармоники (4.31). Тем самым, приравнивая коэффициенты при указанных гармониках к нулю, приходим к счетной системе

$$
\begin{aligned}
\frac{d z_{n, k}}{d \tau}=(1 & \left.-\nu \omega_{n, k}\right) z_{n, k}-\left(1+i c_{0}\right)\left[\frac{9}{4}\left|z_{n, k}\right|^{2}+3 \sum_{\substack{m=1 \\
m \neq n}}^{\infty}\left|z_{m, k}\right|^{2}\right. \\
& \left.+3 \sum_{\substack{m=1 \\
m \neq k}}^{\infty}\left|z_{n, m}\right|^{2}+2 \sum_{\substack{m, r=1 \\
m \neq n, r \neq k}}^{\infty}\left|z_{m, r}\right|^{2}\right] z_{n, k}, \quad n, k=1,2, \ldots,
\end{aligned}
$$

а для $\rho_{n, k}=\left|z_{n, k}\right|^{2}$ после замены $2 \tau \rightarrow \tau$ получаем в точности систему (4.15).

Завершая обсуждение краевой задачи (4.30), отметим, что для нее остается в силе аналог теоремы 4.1. Следовательно, при условии иррациональности отношения $\sigma_{1}^{2} / \sigma_{2}^{2}$ эта задача имеет по параметру $\nu$ ту же динамику, что и задача (4.9), т. е. в ней наблюдается феномен турбулентной буферности.

\section{$\S 5$. Заключение}

Остановимся на связи гипотезы Ландау с так называемым феноменом диффузионного хаоса. Суть этого феномена поясним на примере системы

$$
\dot{w}_{k}+i \mu\left(w_{k+1}-2 w_{k}+w_{k-1}\right)=w_{k}-\left(1+i c_{0}\right)\left|w_{k}\right|^{2} w_{k}, \quad k=1, \ldots, N,
$$


где $w_{k}=w_{k}(t)$ - комплекснозначные функции, $0<\mu \ll 1, c_{0}=$ const $>0$, $w_{N+1}=w_{N}, w_{0}=w_{1}$.

Покажем, что система (5.1) имеет глобально экспоненциально устойчивый $N$-мерный инвариантный тор. Для этого сначала сделаем в ней замены $w_{k}=$ $\rho_{k} \exp \left(i \tau_{k}\right), k=1, \ldots, N$, где $\rho_{k} \geqslant 0,0 \leqslant \tau_{k} \leqslant 2 \pi(\bmod 2 \pi)$. В результате она преобразуется к виду

$$
\begin{gathered}
\dot{\rho}_{k}=\rho_{k}-\rho_{k}^{3}+\mu\left[\rho_{k+1} \sin \alpha_{k}-\rho_{k-1} \sin \alpha_{k-1}\right], \quad k=1, \ldots, N, \\
\dot{\alpha}_{k}=-c_{0}\left(\rho_{k+1}^{2}-\rho_{k}^{2}\right)-\mu\left[\frac{\rho_{k+2}}{\rho_{k+1}} \cos \alpha_{k+1}+\left(\frac{\rho_{k}}{\rho_{k+1}}-\frac{\rho_{k+1}}{\rho_{k}}\right) \cos \alpha_{k}\right. \\
\left.-\frac{\rho_{k-1}}{\rho_{k}} \cos \alpha_{k-1}\right], \quad k=1, \ldots, N-1, \\
\dot{\tau}_{N}=-c_{0} \rho_{N}^{2}-\mu\left[\frac{\rho_{N-1}}{\rho_{N}} \cos \alpha_{N-1}-1\right],
\end{gathered}
$$

где $\tau_{0}=\tau_{1}, \tau_{N+1}=\tau_{N}, \rho_{0}=\rho_{1}, \rho_{N+1}=\rho_{N}, \alpha_{k}=\tau_{k+1}-\tau_{k}$.

Нетрудно заметить, что интересующий нас глобально устойчивый инвариантный тор заведомо существует у системы (5.2)-(5.4) при $\mu=0$. Действительно, в этом случае он задается равенствами $\rho_{k}=1, k=1, \ldots, N$, а поведение траекторий на нем описывают уравнения $\dot{\alpha}_{k}=0, k=1, \ldots, N-1, \dot{\tau}_{N}=-c_{0}$. Далее, из общих результатов монографии [38] следует, что указанный тор сохраняется у системы (5.2)-(5.4) и при всех малых $\mu>0$, причем теперь он имеет вид

$$
\rho_{k}=1+\mu \psi_{k}\left(\alpha_{1}, \ldots, \alpha_{N-1}, \mu\right), \quad k=1, \ldots, N,
$$

где достаточно гладкие по совокупности переменных $2 \pi$-периодические по $\alpha_{s}$, $s=1, \ldots, N-1$, функции $\psi_{k}$ таковы, что

$$
\psi_{k}\left(\alpha_{1}, \ldots, \alpha_{N-1}, 0\right)=\frac{1}{2}\left(\sin \alpha_{k}-\sin \alpha_{k-1}\right), \quad k=1, \ldots, N .
$$

Что же касается движений на данном торе, то они описываются системой

$$
\begin{gathered}
\dot{\alpha}_{k}=\mu \Phi_{k}\left(\alpha_{1}, \ldots, \alpha_{N-1}, \mu\right), \quad \dot{\tau}_{N}=-c_{0}+\mu \Psi\left(\alpha_{1}, \ldots, \alpha_{N-1}, \mu\right), \\
k=1, \ldots, N-1,
\end{gathered}
$$

получающейся из (5.3), (5.4) при учете соотношений (5.5). Отметим еще вытекающие из (5.6) равенства

$$
\begin{gathered}
\left.\Phi_{k}\right|_{\mu=0}=-c_{0}\left(\sin \alpha_{k+1}-2 \sin \alpha_{k}+\sin \alpha_{k-1}\right)+\cos \alpha_{k-1}-\cos \alpha_{k+1}, \\
k=1, \ldots, N-1 .
\end{gathered}
$$

За возможные аттракторы, лежащие на торе (5.5), отвечает, очевидно, отщепляющаяся от (5.7) система для $\alpha_{k}, k=1, \ldots, N-1$. Для удобства последующего численного анализа выполним в ней замену $\mu t \rightarrow t$ и отбросим в правых частях получившейся системы слагаемые порядка малости $\mu$ и выше. В результате с учетом равенств (5.8) она преобразуется к виду

$$
\begin{gathered}
\dot{\alpha}_{k}=-c_{0}\left(\sin \alpha_{k+1}-2 \sin \alpha_{k}+\sin \alpha_{k-1}\right)+\cos \alpha_{k-1}-\cos \alpha_{k+1}, \\
k=1, \ldots, N-1,
\end{gathered}
$$

где $\alpha_{0}=\alpha_{N}=0$. 
Вычисления, проделанные С.Д. Глызиным еще в 1988 г. (см. [39]) и впоследствии уточненные в статье [40], показали, что, например, при $c_{0}=0.6$ и при $N \geqslant 5$ система (5.9) имеет хаотический аттрактор, ляпуновская размерность $d_{L}$ которого при увеличении $N$ растет приблизительно по линейному закону, а именно, $d_{L} \approx 0.763 N-0.568$. Аналогичное справедливо и при других значениях $c_{0}$.

Следует отметить, что система (5.1) служит конечномерным аналогом краевой задачи для уравнения Гинзбурга-Ландау вида

$$
w_{t}+i \mu w_{x x}=w-\left(1+i c_{0}\right)|w|^{2} w,\left.\quad w_{x}\right|_{x=0}=\left.w_{x}\right|_{x=1}=0 .
$$

Поэтому есть все основания ожидать, что в данной задаче реализуется интересующий нас феномен диффузионного хаоса. Последнее означает, что порождаемая задачей (5.10) динамическая система в $\stackrel{\circ}{W} \underset{2}{2}[0,1] \times \stackrel{\circ}{W_{2}^{2}}[0,1]$ имеет хаотический аттрактор, ляпуновская размерность которого неограниченно возрастает при $\mu \rightarrow 0$.

Подведем итог. Разобранные нами содержательные примеры нелинейных динамических систем свидетельствуют о том, что сценарий развития турбулентности по Ландау при условии отказа от требования минимальности возникающих инвариантных торов уже не является экзотическим. Более того, его можно увязать с концепцией диффузионного хаоса, если предположить, что в цепочке бифуркаций (1.62) каждый тор $T_{N}$, начиная с некоторого номера $N_{0} \geqslant 3$, служит носителем хаотического аттрактора и при $N \rightarrow \infty$ ляпуновская размерность этого аттрактора неограниченно растет.

Модифицированный таким способом сценарий Ландау уместно назвать сценарием Ландау-Селла, поскольку принципиальная возможность сохранения хаотического аттрактора при бифуркации $T_{N} \rightarrow T_{N+1}$ была установлена в работе Дж. Селла [10].

Было бы весьма интересно найти конкретный содержательный пример, в котором наблюдается сценарий Ландау-Селла. Пока же ограничимся приведенными выше косвенными соображениями, свидетельствующими о том, что данный сценарий реализуется в модели (5.10) по крайней мере в ослабленном варианте, когда речь идет уже не о торах, а только о неограниченном росте размерности хаотического аттрактора при изменении некоторого управляющего параметра.

\section{Список литературы}

[1] Л.Д. Ландау, "К проблеме турбулентности", Докл. АН СССР, 44:8 (1944), 339-342; англ. пер.: L. D. Landau, "On the problem of turbulence", C. R. Acad. Sci. URSS (N.S.), 44 (1944), 311-314.

[2] E. Hopf, "A mathematical example displaying features of turbulence", Comm. Pure Appl. Math., 1:4 (1948), 303-322.

[3] В. И. Арнольд, Б. А. Хесин, Топологические методы в гидродинамике, доп. изд., МЦНМО, М., 2007; пер. с англ.: V. I. Arnol'd, B. A. Khesin, Topological methods in hydrodynamics, Appl. Math. Sci., 125, Springer-Verlag, New York, 1998.

[4] E. N. Lorenz, "Deterministic nonperiodic flow", J. Atmospheric Sci, 20:2 (1963), $130-141$. 
[5] D. Ruelle, F. Takens, "On the nature of turbulence", Comm. Math. Phys., 20:3 (1971), 167-192.

[6] S. Newhouse, D. Ruelle, F. Takens, "Occurence of strange Axiom A attractors nearquasiperiodic flows on $T^{m}, m \geqslant 3$ ", Comm. Math. Phys., 64:1 (1978), 35-40.

[7] П. Берже, И. Помо, К. Видаль, Порядок в хаосе. О детерминистском подходе $к$ турбулентности, Мир, М., 1991; пер. с франц.: P. Bergé, Y. Pomeau, Ch. Vidal, L'ordre dans le chaos. Vers une approche deterministe de la turbulence, Collection Enseignement des Sciences, 33, Hermann, Paris, 1984.

[8] M. J. Feigenbaum, "Quantitative universality for a class of nonlinear transformations", J. Statist. Phys., 19:1 (1978), 25-52.

[9] Г. В. Осипов, "О развитии турбулентности по Ландау в дискретной модели потоковых систем”, Изв. вузов. Сер. радиофиз., 31:5 (1988), 624-627.

[10] G. R. Sell, "Resonance and bifurcations in Hopf-Landau dynamical systems", Nonlinear dynamics and turbulence, Interaction Mech. Math. Ser., Pitman, Boston, MA, 1983, 305-313.

[11] А. М. Ильин, А. С. Калашников, О.А. Олейник, “Линейные уравнения второго порядка параболического типа", УМH, 17:3 (1962), 3-146; англ. пер.: A. M. Il'in, A.S. Kalashnikov, O. A. Oleinik, "Linear equations of the second order of parabolic type", Russian Math. Surveys, 17:3 (1962), 1-143.

[12] О.А. Ладыженская, В.А. Солонников, Н.Н. Уральцева, Линейные и квазилинейные уравнения параболического типа, Наука, М., 1967; англ. пер.: O. A. Ladyzhenskaya, V.A. Solonnikov, N. N. Ural'tseva, Linear and quasi-linear equations of parabolic type, Transl. Math. Monogr., 23, Amer. Math. Soc., Providence, RI, 1968.

[13] Д. Хенри, Геометрическая теория полулинейных параболических уравнений, Мир, М., 1985; пер. с англ.: D. Henry, Geometric theory of semilinear parabolic equations, Lecture Notes in Math., 840, Springer-Verlag, Berlin-New York, 1981.

[14] Ю. Л. Далецкий, М. Г. Крейн, Устойчивость решений дифференциалъных уравнений в банаховом пространстве, Нелинейный анализ и его приложения, Наука, М., 1970; англ. пер.: Yu. L. Daleckii, M. G. Krein, Stability of solutions of differential equations in Banach space., Transl. Math. Monogr., 43, Amer. Math. Soc., Providence, R.I., 1974.

[15] M. Bartuccelli, P. Constantin, C. R. Doering, J. D. Gibbon, M. Gisselfält, "On the possibility of soft and hard turbulence in the complex Ginzburg-Landau equation", Phys. D, 44:3 (1990), 421-444.

[16] H. Iwasaki, S. Toh, "Statistics and structures of strong turbulence in a complex Ginzburg-Landau equation", Progr. Theoret. Phys., 87:5 (1992), 1127-1137.

[17] Г. Г. Малинецкий, А. Б. Потапов, Современные проблемы нелинейной динамики, Эдиториал УРСС, М., 2002.

[18] А.Ю. Колесов, Н.Х. Розов, “Аттракторы типа жесткой турбулентности в релаксационных системах", Дифферени. уравнения, 38:12 (2002), 1596-1605; англ. пер.: A. Yu. Kolesov, N. Kh. Rozov, "Attractors of hard turbulence type in relaxation systems", Differ. Equ., 38:12 (2002), 1694-1702.

[19] А. Ю. Колесов, Н. Х. Розов, "Переключающая перемежаемость в релаксационных системах", Дифферени. уравнения, 39:1 (2003), 35-44; англ. пер.: А. Yu. Kolesov, N. Kh. Rozov, "On-off intermittency in relaxation systems", Differ. Equ., 39:1 (2003), $36-45$.

[20] А. Ю. Колесов, Н.Х. Розов, В.А. Садовничий, "Жизнь на кромке хаоса", Тр. сем. им. И.Г. Петровского, 23 (2003), 219-266; англ. пер.: A. Yu. Kolesov, N. Kh. Rozov, V. A. Sadovnichiy, "Life on the edge of chaos", J. Math. Sci. (N. Y.), 120:3 (2004), 1372-1398. 
[21] А.Н.Шарковский, Ю. Л. Майстренко, Е. Ю. Романенко, Разностнъе уравнения u ux приложения, Наукова думка, Киев, 1986; англ. пер.: А. N. Sharkovskij, Yu. L. Majstrenko, E. Yu. Romanenko, Difference equations and their applications, Math. Appl., 250, Kluwer, Dordrecht, 1993.

[22] А.Д. Морозов, Введение в теорию фракталов, Институт компьютерных исследований, М., Ижевск, 2002.

[23] Н. В. Николенко, "Инвариантные, асимптотически устойчивые торы возмущенного уравнения Кортевега-де Фриза”, УМH, 35:5 (1980), 121-180; англ. пер.: N. V. Nikolenko, "Invariant asymptotically stable tori of the perturbed Kortewegde Vries equation", Russian Math. Surveys, 35:5 (1980), 139-207.

[24] А. Ю. Колесов, Н.Х. Розов, Инвариантные торы нелинейных волновых уравнений, Физматлит, М., 2004.

[25] Е.Ф. Мищенко, В.А. Садовничий, А. Ю. Колесов, Н.Х. Розов, Автоволновые прочессы в нелинейных средах с диффузией, Физматлит, М., 2005.

[26] Т. Пу, Нелинейная экономическая динамика, Издательский дом "Удмуртский университет", Ижевск, 2000; пер. с англ.: Т. Puu, Nonlinear economic dynamics, Lecture Notes in Econom. and Math. Systems, 336, Springer-Verlag, Berlin, 1989.

[27] В.Б. Занг, Синергетическая экономика. Время и перемены в нелинейной экономической теории, Мир, М., 1999; пер. с англ.: Wei-Bin Zhang, Synergetic economics. Time and change in nonlinear economics, Springer Ser. Synergetics, 53, Springer-Verlag, Berlin, 1991.

[28] J.R. Hicks, A contribution to the theory of the trade cycle, Oxford University Press, Oxford, 1950.

[29] Н.Н. Боголюбов, Ю.А. Митропольский, Асимптотические методы в теории нелинейных колебаний, 3-е изд., Физматлит, М., 1963; нем. пер.: N. N. Bogoljubow, J. A. Mitropolski, Asymptotische Methoden in der Theorie der nichtlinearen Schwingungen, Akademie-Verlag, Berlin, 1965.

[30] Ю.Н. Бибиков, Многочастотные нелинейные колебания и их бифуркации, ЛГУ, Л., 1991.

[31] A. C. Scott, "Distributed multimode oscillators of one and two spatial dimensions", IEEE Trans. Circuits Syst. I Regul. Pap., CT-17:1 (1970), 55-80.

[32] A.C. Scott, "Tunnel diode arrays for information processing and storage", IEEE Trans. Syst., Man, Cybern., SMC-1:3 (1971), 267-275.

[33] Э. Скотт, Нелинейная наука: рождение и развитие когерентных структур, Физматлит, М., 2007; пер. с англ.: А. Scott, Nonlinear science. Emergence and dynamics of coherent structures, Oxf. Texts Appl. Eng. Math., 1, Oxford University Press, Oxford, 1999.

[34] R. D. Parmentier, "Lumped multimode oscillators in the continuum approximation", IEEE Trans. Circuits Syst. I Regul. Pap., CT-19:2 (1972), 142-145.

[35] А. Ю. Колесов, Н.Х. Розов, "Особенности динамики уравнения Гинзбурга-Ландау в плоской области”, ТМФ, 125:2 (2000), 205-220; англ. пер.: A. Yu. Kolesov, N. Kh. Rosov, "Characteristic features of the dynamics of the Ginzburg-Landau equation in a plane domain", Theoret. Math. Phys., 125:2 (2000), 1476-1488.

[36] А.Ю. Колесов, Е.Ф. Мищенко, Н.Х. Розов, "Феномен буферности в нелинейной физике", Тр. МИАН, 250 (2005), 112-182; англ. пер.: А. Yu. Kolesov, E. F. Mishchenko, N. Kh. Rosov, "Buffer phenomenon in nonlinear physics", Proc. Steklov Inst. Math., 250 (2005), 102-168.

[37] Т. С. Ахромеева, С. П. Курдюмов, Г. Г. Малинецкий, А. А. Самарский, "О классификации решений системы нелинейных диффузионных уравнений в окрестности точки бифуркации", Итоги науки и техники. Сер. Соврем. пробл. матем. Нов. достиж., 28, № 316, ВИНИТИ, 1986, 207-313; англ. пер.: Т. S. Akhromeeva, S.P. Kurdyumov, G. G. Malinetskii, A.A. Samarskii, "On the classification of the 
solutions of a system of nonlinear diffusion equations in a neighborhood of a bifurcation point", J. Soviet Math., 41, № 5, 1988, 1292-1358.

[38] Ю.А. Митропольский, О.Б. Лыкова, Интегралънъе многообразия в нелинейной механике, Нелинейный анализ и его приложения, Наука, М., 1973.

[39] С.Д. Глызин, “Численное обоснование гипотезы Ландау-Колесова о природе турбулентности", Математические модели в биологии и медицине, 3, Вильнюс, ИМК, 1989, 31-36.

[40] С. Д. Глызин, А. Ю. Колесов, Н. Х. Розов, "Хаотическая буферность в цепочках связанных осцилляторов", Дифферени. уравнения, 41:1 (2005), 41-49; англ. пер.: S. D. Glyzin, A. Yu. Kolesov, N. Kh. Rozov, "Chaotic buffering property in chains of coupled oscillators", Differ. Equ., 41:1 (2005), 41-49.

А. Ю. Колесов (А. Yu. Kolesov)

Ярославский государственный университет

им. П. Г. Демидова

E-mail: kolesov@uniyar.ac.ru

\section{Н. Х. Розов (N. Kh. Rozov)}

Московский государственный университет им. М. В. Ломоносова

E-mail: rozov@rozov.mccme.ru

В. А. Садовничий (V. A. Sadovnichii)

Московский государственный университет им. М. В. Ломоносова

E-mail: rector@rector.msu.su
Поступила в редакцию 09.01.2008 